\title{
Nonlinear aspects of quantum plasma physics
}

\author{
P K Shukla ${ }^{1,2,3,4,5}$ and B Eliasson ${ }^{1,4,5}$ \\ ${ }^{1}$ Institut für Theoretische Physik IV, Fakultät für Physik und Astronomie, \\ Ruhr-Universität Bochum, D-44780 Bochum, Germany \\ ${ }^{2}$ Scottish Universities Physics Alliance (SUPA, Department of Physics, \\ University of Strathclyde, Glasgow G4 ONG, United Kingdom \\ ${ }^{3}$ Instituto de Plasmas e Fusão Nuclear, Instituto Superior Técnico, \\ Universidade Técnica de Lisboa, 1049-001 Lisboa, Portugal \\ ${ }^{4}$ Department of Physics, Umeå University, SE-90 187 Umeå, Sweden \\ ${ }^{5}$ The Abdus Salam International Centre for Theoretical Physics, I-34014 Trieste, Italy
}

(Received 25 June 2009; Revised 26 August 2009)

\begin{abstract}
Dense quantum plasmas are ubiquitous in planetary interiors and in compact astrophysical objects (e.g. the interior of white dwarf stars, in magnetars etc.), in semiconductors and micro-mechanical systems, as well as in the next generation intense laser-solid density plasma interaction experiments and in quantum x-ray free-electron lasers. In contrast to classical plasmas, one encounters extremely high plasma number density and low temperature in quantum plasmas. The latter are composed of electrons, positrons and holes, which are degenerate. Positrons (holes) have the same (slightly different) mass as electrons, but opposite charge. The degenerate charged particles (electrons, positrons, holes) follow the Fermi-Dirac statistics. In quantum plasmas, there are new forces associated with i) quantum statistical electron and positron pressures, ii) electron and positron tunneling through the Bohm potential, and iii) electron and positron angular momentum spin. Inclusion of these quantum forces provides possibility of very high-frequency dispersive electrostatic and electromagnetic waves (e.g. in the hard x-ray and gamma rays regimes) having extremely short wavelengths. In this review paper, we present theoretical backgrounds for some important nonlinear aspects of wave-wave and wave-electron interactions in dense quantum plasmas. Specifically, we shall focus on nonlinear electrostatic electron and ion plasma waves, novel aspects of 3D quantum electron fluid turbulence, as well as nonlinearly coupled intense electromagnetic waves and localized plasma wave structures. Also discussed are the phase space kinetic structures and mechanisms that can generate quasi-stationary magnetic fields in dense quantum plasmas. The influence of the external magnetic field and the electron angular momentum spin on the electromagnetic wave dynamics is discussed. Finally, future perspectives of the nonlinear quantum plasma physics are highlighted.
\end{abstract}

PACS numbers: 05.30.Fk,52.35.Mw,52.35.Ra,52.35Sb

\section{Contents}

\section{Introduction}

II. Fluid representation of quantum-like models

III. Nonlinear equations for unmagnetized quantum plasmas $\quad[10$

A. Nonlinear Schrödinger-Poisson equations

B. Inclusion of the ion dynamics

IV. Localized electrostatic excitations

A. Dark solitons and vortices associated with EPOs

B. Localized ion wave excitations in quantum plasmas

\section{Quantum fluid turbulence}

\section{Kinetic phase-space structures}

VII. Magnetic fields in quantum plasmas

A. The quantum Weibel instability

B. Dense plasma magnetization by the electromagnetic wave 
A. Electromagnetic spin waves in magnetized plasmas 27

B. Nonlinearly coupled EM waves 28

1. Nonlinear EMHD 28

2. Nonlinear Hall-MHD

C. Stimulated scattering instabilities 30

D. Self-trapped EM waves in a quantum hole 31

IX. Summary and perspectives

A. Derivation of the Vlasov equation from the Wigner equation

B. Derivation of the dispersion relation for the Wigner-Poisson system 37

References 


\section{INTRODUCTION}

The field of quantum plasma physics has a long and diverse tradition [1, 2, 3, 4, 5, and is becoming of increasing current interest [6, 7], motivated by its potential applications in modern technology (e.g. metallic and semiconductor nanostructures-such as metallic nanoparticles, metal clusters, thin metal films, spintronics, nanotubes, quantum well and quantum dots, nano-plasmonic devices, quantum x-ray free-electron lasers, etc.). Due to the recent development of ultrafast spectroscopy techniques, it is now possible to monitor the femtosecond dynamics of an electron gas confined in metallic plasmas. In dense quantum plasmas, the number densities of degenerate electrons and/or positrons are extremely high, and the plasma particles (mainly electrons and positrons) obey Fermi-Dirac statistics.

The quantum degeneracy effects start playing a significant role when the de Broglie thermal wavelength $\lambda_{B}=$ $\hbar /\left(2 \pi m_{e} k_{B} T\right)^{1 / 2}$ for electrons is similar to or larger than the average inter-electron distance $n_{e}^{-1 / 3}$, i.e. when [6, 7 ]

$$
n_{e} \lambda_{B}^{3} \gtrsim 1
$$

or, equivalently, the temperature $T$ is comparable or lower than the electron Fermi temperature $T_{F e}=E_{F} / k_{B}$, where the electron Fermi energy is

$$
E_{F}=\frac{\hbar^{2}}{2 m_{e}}\left(3 \pi^{2}\right)^{2 / 3} n_{e}^{2 / 3}
$$

so that

$$
\chi=\frac{T_{F e}}{T}=\frac{1}{2}\left(3 \pi^{2}\right)^{2 / 3}\left(n_{e} \lambda_{B}^{3}\right)^{2 / 3} \gtrsim 1
$$

Here $\hbar$ is the Planck constant divided by $2 \pi, n_{e}$ is the electron number density, $m_{e}$ is the rest electron mass, and $k_{B}$ is the Boltzmann constant.

When the temperature approaches the electron Fermi temperature $T_{F e}$, one can show, by using the density matrix formalism [8, that the equilibrium electron distribution function changes from the Maxwell-Boltzmann $\propto \exp \left(-E / k_{B} T\right)$ to the Fermi-Dirac distribution $\propto\left(2 / \hbar^{3}\right)\left[\exp \left((E+\mu) / k_{B} T_{F e}\right)+1\right]^{-1}$, where $E$ is the electron kinetic energy and $\mu$ is the chemical potential. In a dense Fermi plasma, the Thomas-Fermi screening radius reads

$$
\lambda_{F}=\frac{V_{F e}}{\sqrt{3} \omega_{p e}}
$$

which is the quantum analogue of the Debye-Hückel radius. Here the electron Fermi speed

$$
V_{F e}=\left(2 E_{F} / m_{e}\right)^{1 / 2}=\frac{\hbar}{m_{e}}\left(3 \pi^{2} n_{e}\right)^{1 / 3}
$$

is the speed of an electron at the Fermi surface.

A measure of the importance of collisions in a dense plasma is the quantum coupling parameter, which is the ratio between the interaction energy $E_{i n t}=e^{2} n_{e}^{1 / 3}$ and the average kinetic energy $E_{k i n}$ of electrons, where $e$ is the magnitude of the electron charge. For a classical plasma, the kinetic energy is $k_{B} T$ and hence we have $\Gamma_{C}=E_{i n t} / k_{B} T$ in the classical case. In a quantum plasma, we have instead $E_{k i n}=E_{F}$, which gives the quantum coupling parameter [6, 7, 9]

$$
\Gamma_{Q}=\frac{E_{i n t}}{E_{F}}=\frac{2}{\left(3 \pi^{2}\right)^{2 / 3}} \frac{m_{e} e^{2}}{\hbar^{2} n_{e}^{1 / 3}} \sim\left(\frac{1}{n_{e} \lambda_{F}^{3}}\right)^{2 / 3} \sim\left(\frac{\hbar \omega_{p e}}{2 k_{B} T_{F e}}\right)^{2} \equiv H^{2}
$$

(where we have left out proportionality constants for the sake of clarity) is analogous to the classical one when $\lambda_{F} \rightarrow \lambda_{D}$. The various plasma regimes are illustrated in Fig. 11, where the straight lines correspond to i) the distinction between the classical and quantum plasmas, $\chi=1$, ii) the limit between collisionless and collisional classical plasmas, $\Gamma_{C}=1$, and iii) the limit between collisionless and collisional quantum plasmas, $\Gamma_{Q}=1$. In Fig. 1. various experimental and naturally occurring plasmas are exemplified. Also indicated in Fig. 1 are the kinetic equations used to model the plasma in each regime. The Vlasov and Wigner equations are thus used to model collisionless plasma in the classical and quantum limits, respectively, while "Boltzmann" indicates collisional kinetic models in a classical plasma. The kinetic models for a collisional quantum plasma is labeled "Wigner (+coll)" in Fig. 1. We note that a quantum plasma becomes collisionless $\Gamma_{Q}<1$ when the mean distance between electrons is of the order of the Bohr radius $a_{0}$, i.e. $d=1 / n_{e}^{1 / 3}<\left[\left(3 \pi^{2}\right)^{2 / 3} / 8 \pi\right] a_{0} \approx 0.38 a_{0}$, where $a_{0}=\hbar^{2} / m_{e} e^{2} \approx 0.53 \AA$. This 
corresponds to a number density of the order $n_{e} \gtrsim 1.22 \times 10^{26} \mathrm{~cm}^{-3}$, which is three orders of magnitude larger than the electron density in a typical metal, for example gold has $n_{e}=5.9 \times 10^{22} \mathrm{~cm}^{-3}$ at room temperature.

In Fermi-degenerate matters, there is an effect called the Pauli-blocking, which strongly reduces the electron-electron and electron-ion collisional rates. Namely, at moderate temperatures, only electrons within an energy shell of thickness $k_{B} T$ about the Fermi surface (where the electron energy equals $E_{F}$ ) can undergo collisions. For these electrons, the electron-electron collision rate is of the order $k_{B} T / \hbar$, and the average collision rate is obtained by multiplying this expression by $T / T_{F}$. The resulting collision frequency $\nu_{e e}$ divided by the electron plasma frequency is 7 ]

$$
\frac{\nu_{e e}}{\omega_{p e}} \sim \frac{E_{F}}{\hbar \omega_{p}}\left(\frac{T}{T_{F}}\right)^{2}=\frac{1}{\Gamma_{Q}^{1 / 2}}\left(\frac{T}{T_{F}}\right)^{2} .
$$

Hence, $\nu_{e e} \ll \omega_{p e}$ when $T \ll T_{F}$ and $\Gamma_{Q}>1$, which is relevant for metallic electrons. For example, at room temperature we have $\nu_{e e} \sim 10^{11} s^{-1}$, which is much smaller than the typical collisionless frequency of collective interactions, $\omega_{p e} \sim 10^{16} \mathrm{~s}^{-1}$. Also, the typical collision rate for electron-lattice (ion) collisions, $\nu_{e i} \sim 10^{15} \mathrm{~s}^{-1}$, is smaller than $\omega_{p e}$ by one order of magnitude. Therefore, a collisionless regime seems to be relevant for free electrons in a metal on a time-scale of the order of a femtosecond. In denser plasmas, such as in stellar interiors [10, 11, 12. and in inertial fusion schemes [13, 14, 15], the relative effects of collisions decrease even further and leads to increased electron transport and heat conductivity.

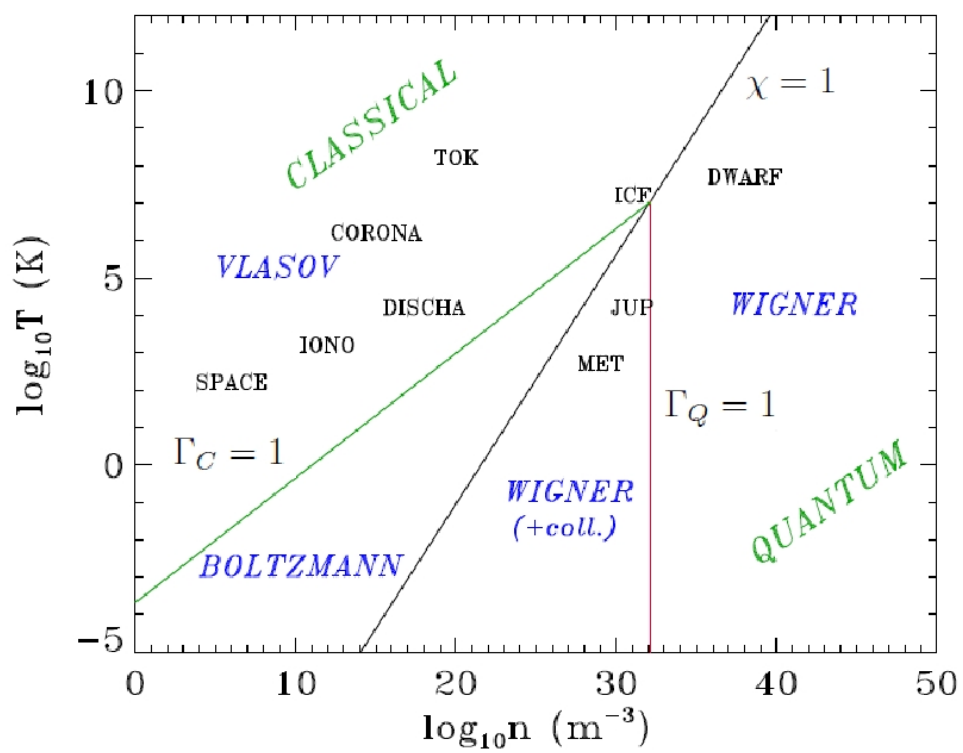

FIG. 1: Schematic plasma diagram in the $\log _{10}(T)-\log (n)$ plane: IONO: ionospheric plasma, SPACE: space plasma, CORONA: solar corona, DISCHA: typical electric discharge, TOK: tokamak/magnetic fusion experiments, ICF: inertial confinement fusion, MET: metals and metal clusters, JUP: Jupiter's core, DWARF: white dwarf star. After Refs. 66, 7].

More than sixty five years ago, Wigner [16] introduced a phase-space formalism to treat a quantum state of charged particles in a collisionless quantum system. He introduced a quantum distribution function of the phase-space variables $f(x, p . t)$. The Wigner distribution function is defined as

$$
f(x, p, t)=\frac{1}{(2 \pi)^{3 N}} \int_{\infty}^{\infty} \rho\left(x-\frac{\hbar}{2} \tau, x+\frac{\hbar}{2} \tau, t\right) \exp (-i p \tau) d \tau,
$$

where $N$ is the number of particles in the system, $x\left\{x ; 1, x_{2} \ldots, x_{N}\right\}$ and $p\left\{p_{1}, p_{2}, \ldots, P_{N}\right\}$ are the sets of coordinates and momenta for the particles, $t$ is the time, and $\rho\left(x, x^{\prime}, t\right)$ is the density matrix. The Wigner function is not a probability density in phase space $x, p$, because it can take negative values. The Wigner distribution function $W_{i}(x, p . t)$, corresponding to the wave function $\psi_{i}(x, t)$, can be expressed as

$$
W_{i}(x, p, t)=\frac{1}{(2 \pi)^{3 N} \hbar} \int_{\infty}^{\infty} d y\left\langle\psi_{i}(x-y / 2, t) \psi_{i}^{*}(x+y / 2, t)\right\rangle \exp (-p y / \hbar)
$$


which has the property

$$
\int_{\infty}^{\infty} d p W_{i}(x, p . t)=<\left|\psi_{i}(x, t)\right|^{2}>
$$

where the asterisk denotes complex conjugate. The quantum kinetic equation based on the Wigner distribution was developed by Moyal [17, and is now referred to as the Wigner-Moyal description for treating statistical effects on electron plasma waves in a quantum plasma [18.

Analytical investigations of collective interactions between an ensemble of degenerate electrons in a dense quantum plasma dates back to early fifties. Specifically, Klimontovich and Silin [1] and Bohm and Pines [2, 3, 4, 5] presented properties of linear electron plasma oscillations (EPOs) in a dense quantum plasma. In the latter, electrons, positrons, and holes are degenerate, while ions are cold and classical (typically, in a dense quantum plasma the ion Fermi speed is much smaller than the electron Fermi speed). Accordingly, electrons, positrons and holes have a Fermi-Dirac distribution function [19], contrary to the Boltzmann-Maxwell distribution function for charged particles in a classical plasma.

The dispersion relation for high-frequency electron plasma oscillations in a dense quantum plasma with fixed ion background reads (see Appendix B)

$$
1-\frac{4 \pi e^{2}}{m_{e}} \int \frac{f_{0}(\mathbf{u})}{(\omega-\mathbf{k} \cdot \mathbf{u})^{2}-\frac{\hbar^{2} k^{4}}{4 m_{e}^{2}}} d^{3} u=0
$$

which was also obtained by Bohm and Pines 3 by performing a series of canonical transformations on the Hamiltonian of the system of individual electrons, interacting via the electrostatic force. In the zero temperature limit, we have (see Appendix B)

$$
\begin{aligned}
& 1+\frac{3 \omega_{p e}^{2}}{4 k^{2} V_{F e}^{2}}\left\{2-\frac{m_{e}}{\hbar k V_{F e}}\left[V_{F e}^{2}-\left(\frac{\omega}{k}+\frac{\hbar k}{2 m_{e}}\right)^{2}\right] \log \left|\frac{\frac{\omega}{k}-V_{F e}+\frac{\hbar k}{2 m_{e}}}{\frac{\omega}{k}+V_{F e}+\frac{\hbar k}{2 m_{e}}}\right|\right. \\
& \left.+\frac{m_{e}}{\hbar k V_{F e}}\left[V_{F e}^{2}-\left(\frac{\omega}{k}-\frac{\hbar k}{2 m_{e}}\right)^{2}\right] \log \left|\frac{\frac{\omega}{k}-V_{F e}-\frac{\hbar k}{2 m_{e}}}{\frac{\omega}{k}+V_{F e}-\frac{\hbar k}{2 m_{e}}}\right|\right\} \equiv 1+\chi_{e}=0,
\end{aligned}
$$

which, in the limit $\hbar k / m_{e} \rightarrow 0$ yields

$$
1+\frac{3 \omega_{p e}^{2}}{k^{2} V_{F e}^{2}}\left(1-\frac{\omega}{2 k V_{F e}} \log \left|\frac{\omega+k V_{F e}}{\omega-k V_{F e}}\right|\right)=0,
$$

where we have assumed that $\omega$ is real and $\omega / k>V_{F e}$. Here $\omega$ is the wave frequency, $\mathbf{k}$ is the wave vector, and $\omega_{p e}=\left(4 \pi n_{e} e^{2} / m_{e}\right)^{1 / 2}$ is the electron plasma frequency, On the other hand, for small wavenumbers up to terms containing $k^{4}$, we obtain from 12

$$
\omega^{2} \approx \omega_{p e}^{2}+\frac{3}{5} k^{2} V_{F e}^{2}+(1+\alpha) \frac{\hbar^{2} k^{4}}{4 m_{e}^{2}},
$$

where $\alpha=(48 / 175) m_{e}^{2} V_{F e}^{4} / \hbar^{2} \omega_{p e}^{2} \approx 2.000\left(a_{0}^{3} n_{0}\right)^{1 / 3}$, where $a_{0}=\hbar^{2} / m_{e} e^{2} \approx 53 \times 10^{-10} \mathrm{~cm}$ is the Bohr radius. Equation (14) shows that the wave dispersion arises due to the finite width of the electron wave function in a dense Fermi plasma [7, 20, 21, 22, 23, 24.

Furthermore, in the low phase speed limit, viz. $\omega \ll k V_{F e}$, the dielectric constant for ion oscillations reads

$$
\epsilon(\omega, \mathbf{k}) \approx 1+\frac{3 \omega_{p e}^{2}}{k^{2} V_{F e}^{2}+3 \hbar^{2} k^{4} / 4 m_{e}^{2}}-\frac{\omega_{p i}^{2}}{\omega^{2}},
$$

which yields, with $\epsilon(\omega, \mathbf{k})=0$, the ion oscillation frequency

$$
\omega \approx \frac{\omega_{p i}}{(1+Q)^{1 / 2}}
$$

where $\omega_{p i}=\left(m_{e} / m_{i}\right)^{1 / 2} \omega_{p e}$ is the ion plasma frequency, and $m_{i}$ is the ion mass, $Q=3 \omega_{p e}^{2} /\left(k^{2} V_{F e}^{2}+3 \hbar^{2} k^{4} / 4 m_{e}^{2}\right)$, and $\alpha \ll 1$. For $Q \gg 1$, we have from $(16 \mathrm{~b})$

$$
\omega \approx k C_{F s}\left(1+\frac{\hbar^{2} k^{4}}{4 m_{e}^{2} \omega_{p e}^{2}}\right)^{1 / 2}
$$


where $C_{F s}=\left(T_{F e} / 3 m_{i}\right)^{1 / 2}$ is the sound speed.

Dispersion properties of electrostatic waves in an unmagnetized dense quantum plasmas with arbitrary electron degeneracy have been presented by Maafa [25] and Melrose [26. By using the random phase approximation, the permittivity of a degenerate collisionless plasma is given in textbooks [27, 28. Furthermore, since the pure electromagnetic wave in a non-streaming unmagnetized dense plasma do not accompany density fluctuations, the wave frequency is $\omega=\left(\omega_{p e}^{2}+k^{2} c^{2}\right)^{1 / 2}$, where $c$ is the speed of light in vacuum. Theoretical studies of quantum statistical properties of dense plasmas in the presence of electromagnetic waves appear in Kremp et al. [29] and in textbooks [30, 31, while quantum parameter regimes are discussed by Bonitz [6]. In a magnetized dense quantum plasma, one finds that the external magnetic field significantly affects the dynamics of degenerate electrons and positrons, and subsequently there appear new collective phenomena associated with the electron angular momentum spin [32, 33, the electron spin magnetic moment [34, and quantized Landau energy levels [35] of the Fermions in a strong magnetic field. It turns out that the thermodynamics and kinetics [36], as well as the dispersion properties of both electrostatic and electromagnetic waves [37, 38, 39, 40, 41, 42, 43] in a quantum magnetoplasma are significantly different from those in an unmagnetized quantum plasma.

It was early recognized that the underlying physics of nonlinear quantum-like equations can be better understood by casting those equations in the form of hydrodynamical (or the Euler) equations, which essentially represent the evolution of quantum particle densities and momenta. This was elegantly done by Madelung [44] and Bohm [2] by introducing an eikonal representation for the wave function evolution in the non-stationary Schrödinger equation. The derivation of the Madelung quantum fluid equations for the Pauli equation with the quantum particle angular momentum spin was presented by Takabayashi [45, 46, 47, Bohm et al. [48, 49], Janossy and Ziegler-Naray [50], and others [51. To incorporate relativistic effects into the quantum fluid formalism, Takabayashi derived the quantum electron fluid equations for the Klein-Gordon equation [52] and for the Dirac equation [53, 54, 55]. Extensions have also been done to fluid descriptions of the Weyl equation for massless spin-1/2 particles (neutrinos) by Bialynicki-Birula [56.

Recently, there has been growing and vibrant interests in investigating new aspects of quantum plasma physics by developing non-relativistic quantum hydrodynamical (QHD) equations [7, 20, 21, 57. The latter include the quantum statistical electron pressure and the quantum force involving tunneling of degenerate electrons through the Bohm potential 20. The Wigner-Poisson (WP) model has also been used to derive a set of non-relativistic quantum hydrodynamical (QHD) equations [7, 21 for a dense electron plasma, assuming immobile ions. The QHD equations are composed of the electron continuity, electron momentum and Poisson equations. The quantum force [7, 20, 21] appears in the non-relativistic electron momentum equation through the pressure term, which requires knowledge of the Wigner distribution for a quantum mixture of electron wave functions, each characterized by an occupation probability. Quantum transport models similar to the QHD plasma model have also been used in superfluidity 58 and superconductivity [59], as well as in the study of metal clusters and nanoparticles, where they are referred to as nonstationary Thomas-Fermi models 60 .

The electrostatic QHD equations are useful for studying collective interactions (e.g. different types of waves, instabilities, quantum fluid turbulence and nonlinear structures [7, 21, 22, 61, 62, 63, 64, 65, 66, 67, 68, 69] in dense quantum plasmas. The quantum kinetic and QHD equations have also been generalized to include the electromagnetic, ambient magnetic field and electron angular momentum spin effects [40, 70, 71, 72, 73, 74. The latter give rise to high-frequency spin waves, which can be excited by neutrino beams in supernovae [70, 75]. Furthermore, studies of numerous collective interactions in dense plasmas are relevant in the context of i) intense laser-solid density plasma experiments [13, 14, 76, 77, 78, 79, 80, 81, 82, 83, 84, 85, where one would be exploring new frontiers in high-energy density physics [86; ii) in the cores of giant planets and the crusts of old stars [87, 88, 89]; iii) superdense astrophysical objects [90, 91, 92, 93, 94, 95, 96, 97, 98, (e.g. interiors of white dwarfs and magnetospheres of neutron stars and magnetars); iv) as well as for micro and nano-scale objects (e.g. quantum diodes 199, 100, 101, 102, 103, 104, quantum dots and nanowires [105], nano-photonics [106, 107, plasmonics [108, ultra-small electronic devices [109, 110, 111], and metallic nanostructures [112]); v) micro-plasmas [113, and quantum x-ray free-electron lasers [114, 115]. Furthermore, it should be stressed that a Fermi degenerate dense plasma may also arise when a pellet of hydrogen is compressed to many times the solid density in the fast ignition scenario for inertial confinement fusion [15, 116, 117. Since there is an impressive developments in the field of short pulse petawatt laser technology, it is highly likely that such plasma conditions can be achieved by intense laser pulse compression using powerful x-ray pulses. Here ultrafast x-ray Thomson scattering techniques can be used to measure the features of laser enhanced plasma lines, which will, in turn, give invaluable informations regarding the equation of state of shock compressed dense matters. Recently, spectrally resolved x-ray scattering measurements [82, 84 have been performed in dense plasmas allowing accurate measurements of the electron velocity distribution function, temperature, ionization state, and of plasmons in the warm dense matter regime [118. This novel technique promises to access the degenerate, the closely coupled, and the ideal plasma regime, making it possible to investigate extremely dense states of matter, such as the inertial confinement fusion fuel during compression, reaching super-solid densities. 
In this review article, we present the theoretical progress that has been recently made in the area of collective nonlinear interactions in collisionless dense quantum plasmas. The manuscript is organized in the following fashion. In section 2, we shall briefly recapitulate the hydrodynamic representation of some quantum-like models that appear in different branches of physics. The governing equations for nonlinearly interacting electrostatic waves in an unmagnetized quantum plasma are derived in section 3. Section 4 presents numerical studies of nonlinear electron plasma wave excitations in the form of quantized one-dimensional dark solitons and quantized two-dimensional vortices. The model used here is the nonlinear Schrödinger equation for the dispersive EPOs, coupled with the Poisson equation for the electrostatic potential. This model is also used for studying 3D quantum electron fluid turbulence in section V] where we find non-Kolmogorov-type turbulence spectra. In section VI, we present recent results concerning the phase space (kinetic) turbulence, by using the Wigner and Vlasov models for the electron distribution function. A theoretical model for the generation of quasi-stationary magnetic fields in a dense quantum plasma due to the Weibel instability is presented in section VIIA. The magnetization of a dense plasma in the presence of a large amplitude electromagnetic wave is demonstrated in subsection VII B. The dynamics of electromagnetic waves in a dense magnetoplasma are discussed in section VIII. Here we focus on spin waves propagating across the magnetic field direction, and develop nonlinear equations for low-phase speed (in comparison with the speed of light) electromagnetic waves in a dense quantum magnetoplasma. Finally, section IX highlights our main results and describes the future prospectives of the quantum plasma physics research.

\section{FLUID REPRESENTATION OF QUANTUM-LIKE MODELS}

This section is included to show how different types of quantum-like models can be cast in the form of hydrodynamic equations.

First, we consider the non-stationary nonlinear Schrödinger equation(NLSE)

$$
i \hbar \frac{\partial \psi}{\partial t}+\frac{\hbar^{2}}{2 m} \nabla^{2} \psi-U_{0}\left(|\psi|^{2}\right) \psi=0,
$$

where $\psi(\mathbf{r}, t)$ is the macroscopic wave function, $m$ is the particle mass, and $U_{0}\left(|\psi|^{2}\right)$ is an effective potential. The NLSE also arises in various physical context in the description of amplitude modulated nonlinear waves in fluids [119, 120, in transmission lines [121, in nonlinear optics for ultra-fast communications [122, 123, 124, in plasmas [125, 126, 127, 128, 129, 130], and in many other areas of physics [131, 132, 133].

Introduce the Madelung transformation 44 ]

$$
\psi(\mathbf{r}, t)=\sqrt{n} \exp \left(i \frac{\varphi_{q}}{\hbar}\right)
$$

where $n$ and $\varphi_{q}$ are real, and obtain from (17) a pair of quantum hydrodynamic equations composed of the continuity and momentum equations, respectively,

$$
\frac{\partial n}{\partial t}+\nabla \cdot(n \mathbf{v})=0
$$

and

$$
m\left(\frac{\partial}{\partial t}+\mathbf{v} \cdot \nabla\right) \mathbf{v}=-\nabla\left[U_{0}(n)+U_{B}\right]
$$

Here $n=n(\mathbf{r}, t)=|\psi|^{2}$ corresponds to the local density per unit length, and $\hbar \nabla \varphi_{q}(\mathbf{r}, t)=m \mathbf{v}$. The quantum potential is

$$
U_{B}=-\frac{\hbar^{2}}{2 m} \frac{\nabla^{2} \sqrt{n}}{\sqrt{n}}
$$

We note that the quantum particle number density $n$ and the quantum velocity field $\mathbf{v}$ can be written as, respectively,

$$
n(\mathbf{r}, t)=\psi \psi^{*} \equiv|\psi|^{2},
$$

and

$$
\mathbf{v}=\frac{\hbar}{2 i m} \frac{\left(\psi^{*} \nabla \psi-\psi \nabla \psi^{*}\right)}{|\psi|^{2}}=-\frac{i \hbar}{2 m} \nabla\left[\ln \left(\frac{\psi}{\psi^{*}}\right)\right]
$$


where the asterisk denotes the complex conjugate. The quantum-like velocity, given by (23), is a potential field, namely,

$$
\nabla \times \mathbf{v}=0
$$

everywhere in a single-connected region.

Let us now define the generalized vorticity on the weighted velocity field as 68.

$$
\boldsymbol{\Omega}=\frac{\nabla \times\left(|\psi|^{2} \mathbf{v}\right)}{|\psi|^{2}}=\nabla \times \mathbf{v}+\frac{\nabla|\psi|^{2} \times \mathbf{v}}{|\psi|^{2}}
$$

where the first term in the right-hand side in (25) represents the ordinary vorticity. It is well known [51, 134] that in the condensate state all rotational flow is carried by quantized vortices (the circulation of the velocity around the core of each such vortex is quantized). In the absence of quantized vortices, the first term is zero in view of (24). In such a situation, the second term in (25) determines the generalized vorticity. Various aspects of quantized vortex dynamics and superfluid turbulence appear in Barenghi et al [134. Bewley et al [135] have presented a technique for visualization of quantized vortices in liquid helium.

Second, the nonlinear Schrödinger equation can be generalized by including the trapping potential

$$
V_{b}(x, y, z)=\frac{1}{2} m_{b}\left(\omega_{x}^{2} x^{2}+\omega_{y}^{2} y^{2}+\omega_{z}^{2} z^{2}\right)
$$

which confines identical bosons [136] in the harmonic trap of an ultracold quantum system. Here $m_{b}$ is the boson mass, and $\omega_{x}, \omega_{y}$ and $\omega_{z}$ are the harmonic frequencies of the bosons along the $x, y$, and $z$ directions, respectively, of a Cartesian coordinate system. The nonlinear dynamics of the Bose-Einstein condensates (BECs) [136, 137] is then governed by the Gross-Pitaevskii equation [138, 139, 140]

$$
i \hbar \frac{\partial \phi(\mathbf{r}, t)}{\partial t}+\frac{\hbar^{2}}{2 m} \nabla^{2} \psi(\mathbf{r}, t)-V_{b} \psi(\mathbf{r}, t)-G|\psi|^{2}(\mathbf{r}, t) \psi(\mathbf{r}, t)=0
$$

where for the BECs we have $U_{0}\left(|\psi|^{2}\right)=\left(4 \pi \hbar^{2} a_{s} / m_{b}\right)^{1 / 2}|\psi|^{2} \equiv G|\psi|^{2}$. Here $m_{b}$ is the mass of the bosons and $a_{s}$ is the scattering length for boson-boson collisions. The BECs are repulsive (attractive) for $G>(<) 0$.

Introducing the Ansatz $\psi(\mathbf{r}, t)=\sqrt{n_{b}(\mathbf{r}, t)} \exp \left[\varphi_{b}(\mathbf{r}, t)\right]$ in $(27)$, we obtain generalized quantum hydrodynamic equations [141, 142 ]

$$
\frac{\partial n_{b}}{\partial t}+\nabla \cdot\left(n \mathbf{u}_{b}\right)=0
$$

and

$$
m_{b} \frac{\partial \mathbf{u}_{b}}{\partial t}=-\nabla\left(V_{b}++\frac{m_{b}}{2} u_{b}^{2}+G n_{b}-\frac{\hbar^{2}}{2 m_{b} \sqrt{n_{b}}} \nabla^{2} \sqrt{n_{b}}\right)
$$

where the particle flux

$$
n_{b}(\mathbf{r}, t) \mathbf{u}_{b}(\mathbf{r}, t)=\frac{\hbar^{2}}{2 i m_{b}}\left(\psi^{*} \nabla \psi-\psi \nabla \psi^{*}\right)
$$

with $\mathbf{u}_{b}=\left(\hbar / m_{b}\right) \nabla \varphi_{b}(\mathbf{r}, t)$. Equation (29) establishes the irrotational nature of the superfluid motion of the BECs. Equations (29) and (30) can be used to study the linear and nonlinear properties of BECs.

Next, we consider the dynamics of a non-relativistic single Fermi $(1 / 2-$ spin) particle (a degenerate electron) governed by the Pauli equation [143, 144]

$$
i \hbar \frac{\partial \Psi}{\partial t}+\frac{\hbar^{2}}{2 m_{e}} \nabla^{2} \Psi-\left[\frac{i e \hbar}{2 m_{e} c}(\mathbf{A} \cdot \nabla+\nabla \cdot \mathbf{A})+\frac{e^{2} \mathbf{A}^{2}}{2 m_{e} c^{2}}-e \phi-\mu_{e} \sigma \cdot \mathbf{B}\right] \Psi=0
$$

where $\Psi(\mathbf{r}, t, \boldsymbol{\sigma})$ is the wave function of the single particle species having the spin $\mathbf{s}=1 / 2 \boldsymbol{\sigma}, \boldsymbol{\sigma}$ is the Pauli spin matrices, $\mathbf{A}$ is the vector potential, $\phi$ is the scalar potential, $\mathbf{B}=\nabla \times \mathbf{A}$, and $\mu_{B}=e \hbar / 2 m_{e} c$ is the Bohr magneton. 
By using the Madelung representation for the complex wave function 145

$$
\Psi(\mathbf{r}, t, \boldsymbol{\sigma})=s \sqrt{n_{e}(\mathbf{r}, t, \boldsymbol{\sigma})} \exp \left[\frac{i S_{e}(\mathbf{r}, t, \boldsymbol{\sigma})}{\hbar}\right],
$$

one can obtain from (31) the quantum magnetohydrodynamic equation [19, 145]

$$
\frac{\partial n_{e}}{\partial t}+\nabla \cdot\left(\frac{n \mathbf{p}_{e}}{m_{e}}\right)=0
$$

and

$$
\left(\frac{\partial}{\partial t}+\frac{1}{m_{e}} \mathbf{p}_{e} \cdot \nabla\right) \mathbf{p}_{e}=e\left[\nabla \phi+\frac{1}{c} \frac{\partial \mathbf{A}}{\partial t}-\frac{1}{c} \mathbf{v}_{e} \times(\nabla \times \mathbf{A})\right]+\frac{\hbar^{2}}{2 m_{e}} \nabla\left(\frac{\nabla^{2} \sqrt{n_{e}}}{\sqrt{n_{e}}}\right)+\mu_{B} \nabla(\boldsymbol{\sigma} \cdot \mathbf{B}),
$$

where $s$ mimics the spinor through which the electron-1/2 spin properties are mediated, $n_{e}(\mathbf{r}, t, \boldsymbol{\sigma})=\Psi \Psi^{*}$ represents the probability density of finding the single electron at some point with a spin $\mathbf{s}$. We have denoted the generalized electron momentum $\mathbf{p}_{e}=\nabla S_{e}-i \hbar s^{*} \nabla s+(e / c) \mathbf{A}$.

We can now express the quantum electron velocity [145]

$$
\mathbf{v}_{e}=\frac{\hbar}{2 m_{e}} \frac{\left(\Psi^{*} \nabla \Psi-\Psi \nabla \Psi^{*}\right)}{|\Psi|^{2}}+i \frac{\hbar}{m_{e}} s^{*} \nabla s-\frac{e}{m_{e} c} \mathbf{A},
$$

the spin density vector

$$
\mathbf{s}=\frac{\hbar}{2} s^{*} \boldsymbol{\sigma} s
$$

and the spin vector transport equation [45]

$$
\frac{d \mathbf{s}}{d t}=\frac{e}{m_{e} c}(\mathbf{s} \times \mathbf{B})+\frac{1}{m_{e} n_{e}}\left[\mathbf{s} \times \frac{\partial}{\partial x_{k}}\left(n_{e} \frac{\partial \mathbf{s}}{\partial x_{k}}\right)\right],
$$

where we have used the summation convention for repeated indices, and have denoted $d / d t \equiv(\partial / \partial t)+\mathbf{v}_{e} \cdot \nabla$ is the total derivative. The electron momentum and electromagnetic fields are coupled via the Maxwell equations.

Ignoring the electromagnetic fields and the particle spin, one can obtain, after linearizing (33) and (34), the frequency of electron oscillations

$$
\omega_{g}=\frac{\hbar k^{2}}{2 m_{e}}
$$

where $k$ is the wave number.

Finally, we consider interaction of an electron with both background electrons and singly charged positive ions. The electron dynamics is governed by [146]

$$
i \hbar \frac{\partial \psi}{\partial t}+\frac{\hbar^{2}}{2 m_{e}} \nabla^{2} \psi-U\left(|\psi|^{2}\right)=0
$$

where

$$
\left.U\left(|\psi|^{2}\right)=\left.e^{2} \int d^{3} \mathbf{r}^{\prime} \frac{1}{\left|\mathbf{r}-\mathbf{r}^{\prime}\right|}\left(\mid \psi\left(\mathbf{r}^{\prime}, t\right)\right)\right|^{2}-n_{i}\left(\mathbf{r}^{\prime}, t\right)\right),
$$

is the potential responsible for the interaction of an electron with background matter which includes electrons and positively charged ions with the number density $n_{i}\left(\mathbf{r}^{\prime}, t\right)$. The wave function is normalized on the number density of electrons, viz. $n_{e}(\mathbf{r}, t)=|\psi(\mathbf{r}, t)|^{2}$. We note that Eq. (39) accounts only for the Coulomb interactions between electrons and ions, and completely ignores the quantum statistical pressure, the self-consistent ambipolar field arising from the charge separation, and the electron spin-1/2 effect. 
Assume that $\psi(\mathbf{r}, t)=\psi_{0}+\psi_{1}(\mathbf{r}, t)$, where $\left|\psi_{0}\right|^{2}=n_{0}$ represent the unperturbed electron number density and the perturbation wave function $\psi_{1}(\mathbf{r}, t)$ for spherically symmetric oscillations has the form $\left(A_{k} / r\right) \sin (\mathrm{kr}) \exp (-i \omega t)$, with $A_{k}$ being the normalization constant. Thus, the dispersion relation deduced from (39) reads [146]

$$
k^{2}=\frac{\omega m_{e}}{\hbar}\left[1 \pm\left(1-4 \frac{\omega_{p e}^{2}}{\omega^{2}}\right)^{1 / 2}\right]
$$

The interaction between two electrons participating in spherically symmetric electron oscillations has been considered in Ref. 146. The latter predicts that there would be an effective attraction between electrons mediated by low energy [given by the minus sign in Eq. (41)] spherically symmetric oscillations of electrons in a quantum plasma. We can thus have attracting degenerate electrons in dense plasmas. The underlying physics of electron attraction here seems to be similar to that of the Cooper pairing of electrons in superconductors in which electrons close to the Fermi level attract each other due to their interactions with crystal lattice vibrations (phonon oscillations). The pairs of electrons act more like bosons which can condensate into the same energy level, contrary to single electrons which are fermions and must obey the Pauli exclusion principle.

\section{NONLINEAR EQUATIONS FOR UNMAGNETIZED QUANTUM PLASMAS}

In the preceding section, we have seen that the quantum Madelung fluid description predicts a diffraction pattern of a single electron or positron. However, collective interactions between an ensemble of degenerate electrons (Fermions) in dense plasmas are responsible for new linear and nonlinear waves and structures.

The quantum $N$-body problem is governed by the Schrödinger equation for the $N$-particle wave function $\psi\left(q_{1}, q_{2}, \ldots, q_{N}, t\right)$, where $q_{j}=\left(\mathbf{r}_{j}, s_{j}\right)$ is the coordinate (space, spin) of particle $j$. For identical Fermions, the equilibrium $N$-particle wave function is given by the Slater determinant [8]

$$
\psi\left(q_{1}, q_{2}, \ldots, q_{N}, t\right)=\frac{1}{\sqrt{N !}}\left|\begin{array}{cccc}
\psi_{1}\left(q_{1}, t\right) & \psi_{2}\left(q_{1}, t\right) & \cdots & \psi_{N}\left(q_{1}, t\right) \\
\psi_{1}\left(q_{2}, t\right) & \psi_{2}\left(q_{2}, t\right) & \cdots & \psi_{N}\left(q_{2}, t\right) \\
\vdots & \vdots & \ddots & \vdots \\
\psi_{1}\left(q_{N}, t\right) & \psi_{2}\left(q_{N}, t\right) & \cdots & \psi_{N}\left(q_{N}, t\right)
\end{array}\right|
$$

which is anti-symmetric under odd numbers of permutations. Hence, $\psi$ vanishes if two rows are identical, which is an expression of the Pauli exclusion principle that two identical Fermions cannot occupy the same state. Example $(N=2): \psi\left(q_{1}, q_{2}, t\right)=\frac{1}{\sqrt{2}}\left[\psi_{1}\left(q_{1}, t\right) \psi_{2}\left(q_{2}, t\right)-\psi_{1}\left(q_{2}, t\right) \psi_{2}\left(q_{1}, t\right)\right]$ so that $\psi\left(q_{2}, q_{1}, t\right)=-\psi\left(q_{1}, q_{2}, t\right)$ and $\psi\left(q_{1}, q_{1}, t\right)=0$. Due to the Pauli exclusion principle, all electrons are not permitted to occupy the lowest energy state, and in the ultra-cold limit when all energy states up to the Fermi energy level are occupied by electrons, there is still a quantumstatistical pressure determined by the Fermi pressure.

To describe collective electrostatic oscillations in a plasma, the quantum analogue of the Vlasov-Poisson system is the Wigner-Poisson system, given by

$$
\frac{\partial f}{\partial t}+\mathbf{v} \cdot \nabla f=-\frac{i e m_{e}^{3}}{(2 \pi)^{3} \hbar^{4}} \iint e^{i m_{e}\left(\mathbf{v}-\mathbf{v}^{\prime}\right) \cdot \boldsymbol{\lambda} / \mathbf{h}}\left[\phi\left(\mathbf{x}+\frac{\boldsymbol{\lambda}}{2}, t\right)-\phi\left(\mathbf{x}-\frac{\boldsymbol{\lambda}}{2}, t\right)\right] f\left(\mathbf{x}, \mathbf{v}^{\prime}, t\right) d^{3} \lambda d^{3} v^{\prime},
$$

and

$$
\nabla^{2} \phi=4 \pi e\left(\int f d^{3} v-n_{0}\right)
$$

assuming immobile ions. We note (see Appendix A) that the Wigner equation converges to the Vlasov equation for classical particles (electrons) when $\hbar \rightarrow 0$

$$
\frac{\partial f}{\partial t}+\mathbf{v} \cdot \nabla f=-\frac{e}{m_{e}} \nabla \phi \cdot \frac{\partial f}{\partial \mathbf{v}}
$$

We now take the moments of the Wigner equation 43 and obtain [up to $O\left(\hbar^{2}\right)$ ] the non-relativistic quantumelectron fluid (or the quantum Madelung fluid) equations [7, 21] composed of the electron continuity equation

$$
\frac{\partial n_{e}}{\partial t}+\nabla \cdot\left(n_{e} \mathbf{u}_{e}\right)=0
$$


the electron momentum equation including the quantum statistical pressure and the quantum force

$$
m_{e}\left(\frac{\partial}{\partial t}+\mathbf{u}_{e} \cdot \nabla\right) \mathbf{u}_{e}=e \nabla \phi-\frac{1}{n_{e}} \nabla P_{e}+\mathbf{F}_{Q},
$$

where $\phi$ is determined from the Poisson equation

$$
\nabla^{2} \phi=4 \pi e\left(n_{e}-n_{0}\right) .
$$

For the degenerate Fermi-Dirac distributed plasma, one has (up to constants of order unity) the quantum statistical pressure for the electrons

$$
P_{e}=\frac{m_{e} V_{F e}^{2} n_{0}}{3}\left(\frac{n_{e}}{n_{0}}\right)^{(D+2) / D}
$$

where $D$ is the number of degrees of freedom in the system. It should be noted that Eliasson and Shukla 69] and Tsintsadze and Tsintsadze [19] have obtained different expressions for $P_{e}$ in the non-zero limit of the electron Fermi temperature.

The quantum force [20] due to electron tunneling through the Bohm potential is

$$
\mathbf{F}_{Q}=\frac{\hbar^{2}}{2 m_{e}} \nabla\left(\frac{\nabla^{2} \sqrt{n_{e}}}{\sqrt{n_{e}}}\right) \equiv-\nabla \phi_{B}
$$

where $\phi_{B}$ represents the Bohm potential. We note that the $\alpha$-term [cf. (14)] does not appear in (50) due to consideration of the term up to $O\left(\hbar^{2}\right)$ in the expansion parameter, as it also happens when the mean-field approximation [20] is used in deducing $\mathbf{F}_{Q}$.

\section{A. Nonlinear Schrödinger-Poisson equations}

By introducing the wave function

$$
\psi(\mathbf{r}, t)=\sqrt{n_{e}(\mathbf{r}, t)} \exp \left(i \varphi_{e}(\mathbf{r}, t) / \hbar\right),
$$

where $S$ is defined according to $m_{e} \mathbf{u}_{e}=\nabla \varphi_{e}$ and $n_{e}=|\psi|^{2}$, it can be shown that the QHD equations [e.g. Eqs. (46)-(48)] are equivalent to the generalized NLS-Poisson system [7, 21]

$$
i \hbar \frac{\partial \psi}{\partial t}+\frac{\hbar^{2}}{2 m_{e}} \nabla^{2} \psi+e \phi \psi-\frac{m_{e} V_{F e}^{2}}{2 n_{0}^{2}}|\psi|^{4 / D} \psi=0
$$

and

$$
\nabla^{2} \phi=4 \pi e\left(\left|\psi^{2}\right|-n_{0}\right)
$$

The derivation of (52) required the electron plasma flow velocity to be curl free everywhere in a singly-connected region, except at points where the electron number density vanishes. This is obviously not valid in general. Similar to quantum-fluid treatment, one should include the generalized electron vorticity $\boldsymbol{\Omega}_{e}=\nabla \times\left(|\psi|^{2} \mathbf{u}_{e}\right) /|\psi|^{2}$, which is non-vanishing [see the discussions below Eq. (25)]. Equation 52 captures the two main properties of a quantum plasma, namely the quantum statistical pressure (fully nonlinear) and quantum dispersion effects, and is coupled selfconsistently to the electrostatic potential via the Poisson equation (53). We thus have a nonlocal nonlinear interaction between the electron density and the electrostatic potential. Furthermore, we note that one-dimensional version of Eq. (52) without the $\phi$-term has also been used to describe the behavior of a Bose-Einstein condensate [147] in the absence of the confining potential.

Linearization of the NLS-Poisson Equations yields the frequency of the EPOs [1, 2, 4, 5]

$$
\omega_{k}=\left(\omega_{p e}^{2}+k^{2} V_{F e}^{2}+\frac{\hbar^{2} k^{4}}{4 m_{e}^{2}}\right)^{1 / 2} .
$$

One can identify two distinct dispersive effects from (54): One long wavelength regime, $V_{F e} \gg \hbar k / 2 m_{e}$, and one short wavelength regime, $V_{F e} \lesssim \hbar k / 2 m_{e}$, separated by a critical wavenumber $k_{\text {crit }}=2 \pi / \lambda_{\text {crit }}=\pi \hbar / m_{e} V_{F e} \sim n_{e}^{-1 / 3}$. 


\section{B. Inclusion of the ion dynamics}

The dynamics of low-phase speed (in comparison with the electron Fermi speed) nonlinear electrostatic ion oscillations in a quantum electron-ion plasma is governed by the inertialess electron equation of motion

$$
0=e \nabla \phi-\frac{1}{n_{e}} \nabla P_{e}+\mathbf{F}_{Q}
$$

the ion continuity

$$
\frac{\partial n_{i}}{\partial t}+\nabla \cdot\left(n_{i} \mathbf{u}_{i}\right)=0
$$

the ion momentum equation

$$
m_{i} n_{i}\left(\frac{\partial}{\partial t}+\mathbf{u}_{i} \cdot \nabla\right) \mathbf{u}_{i}=-Z_{i} n_{i} e \nabla \phi
$$

and the Poisson equation

$$
\nabla^{2} \phi=4 \pi e\left(n_{e}-Z_{i} n_{i}\right)
$$

where $n_{i}$ is the ion number density, $\mathbf{u}_{i}$ is the ion fluid velocity, and $Z_{i}$ is the ion charge state.

In the quasi-neutral approximation, viz. $n_{i}=Z_{i} n_{i}=n$, we can combine Eqs. (55) and (57) to obtain

$$
\left(\frac{\partial}{\partial t}+\mathbf{u}_{i} \cdot \nabla\right) \mathbf{u}_{i}=-\frac{C_{F s}^{2} n_{0}}{n_{i}} \nabla\left(\frac{Z_{i} n_{i}}{n_{0}}\right)^{(D+2) / D}+\frac{\hbar^{2}}{2 m_{e} m_{i}} \nabla\left(\frac{\nabla^{2} \sqrt{Z_{i} n_{i}}}{\sqrt{Z_{i} n_{i}}}\right)
$$

Equations (56) and 59 are the desired set for studying nonlinear ion waves [61, 62, in a dense quantum plasma.

\section{LOCALIZED ELECTROSTATIC EXCITATIONS}

We are now in a position to discuss nonlinear properties and the dynamics of localized electrostatic excitations in a dense quantum plasma based on the nonlinear equations we have developed in the preceding section.

\section{A. Dark solitons and vortices associated with EPOs}

In this subsection, we shall discuss the formation of one-dimensional quantized dark solitons and two-dimensional (2D) quantized vortices associated with the EPOs in a dense quantum plasma at nanoscales (of the order of $V_{F e} / \omega_{p e}$ ). We note that the dynamics of dark solitons in the 2D nonlinear Schrödinger equation in a defocusing medium has been studied by Ivonin [148] and Ivonin et al. [149]. However, for studying the formation and dynamics of electrostatic nanostructures in a dense quantum plasma, we shall use the nonlinear Schrödinger-Poisson equations [22]

$$
i \frac{\partial \Psi}{\partial t}+A \nabla^{2} \Psi+\varphi \Psi-|\Psi|^{4 / D} \Psi=0
$$

and

$$
\nabla^{2} \varphi=|\Psi|^{2}-1
$$

where the normalized wave function $\Psi$ is $\psi / \sqrt{n_{0}}$, the normalized $\varphi$ is $e \phi / k_{B} T_{F e}$, and the time and space variables are in units of $\hbar / k_{B} T_{F e}$ and $V_{F e} / \omega_{p e}$, respectively. We have denoted $A=4 \pi m_{e} e^{2} / \hbar^{2} n_{0}^{1 / 3}$ (see Ref. [22]).

The system of equations (60) and (61) is supplemented by the Maxwell equation

$$
\frac{\partial \mathbf{E}}{\partial t}=i A\left(\Psi \nabla \Psi^{*}-\Psi^{*} \nabla \Psi\right),
$$

where the electric field is $\mathbf{E}=-\nabla \phi$. 
The system $60-62$ has the following conserved integrals: the number of electrons

$$
N=\int|\Psi| d^{3} x
$$

the electron momentum

$$
\mathbf{P}=-i \int \Psi^{*} \nabla \Psi d^{3} x
$$

the electron angular momentum

$$
\mathbf{L}=-i \int \Psi^{*} \mathbf{r} \times \nabla \Psi d^{3} x
$$

and the total energy

$$
\mathcal{E}=\int\left[-\Psi^{*} A \nabla^{2} \Psi+|\nabla \varphi|^{2} / 2+|\Psi|^{2+4 / D} D /(2+D)\right] d^{3} x .
$$

The conserved quantities are required to check the accuracy of the numerical integration of (60) and (61).

For quasi-stationary one-dimensional structures moving with a constant speed $v_{0}$, one can find localized, solitary wave solutions by introducing the ansatz $\Psi=W(\xi) \exp (i K x-i \Omega t)$, where $W$ is a complex-valued function of the argument $\xi=x-v_{0} t$, and $K$ and $\Omega$ are a constant wavenumber and the frequency shift, respectively. By the choice $K=v_{0} / 2 A$, the coupled system of equations can be written as

$$
\frac{d^{2} W}{d \xi^{2}}+\lambda W+\frac{\varphi W}{A}-\frac{|W|^{4} W}{A}=0
$$

and

$$
\frac{d^{2} \varphi}{d \xi^{2}}=|W|^{2}-1
$$

where $\lambda=\Omega / A-v_{0}^{2} / 4 A^{2}$ is an eigenvalue of the system. From the boundary conditions $|W|=1$ and $\varphi=0$ at $|\xi|=\infty$, we determine $\lambda=1 / A$ and $\Omega=1+v_{0}^{2} / 4 A$. The system of Eqs. (67) and (68) admits a first integral in the form

$$
\mathcal{H}=A\left|\frac{d W}{d \xi}\right|^{2}-\frac{1}{2}\left(\frac{d \varphi}{d \xi}\right)^{2}+|W|^{2}-\frac{|W|^{6}}{3}+\varphi|W|^{2}-\varphi-\frac{2}{3}=0
$$

where the boundary conditions $|W|=1$ and $\varphi=0$ at $|\xi|=\infty$ have been employed.

Figure 2 shows profiles of $|W|^{2}$ and $\varphi$ obtained numerically from $(67)$ and $\sqrt{68}$ for a few values of $A$, where $W$ was set to -1 on the left boundary and to +1 on the right boundary, i.e. the phase shift is 180 degrees between the two boundaries. The solutions are in the form of dark solitons, with a localized depletion of the electron density $N_{e}=|W|^{2}$, associated with a localized positive potential. Larger values of the parameter quantum coupling parameter $A$ give rise to larger-amplitude and wider dark solitons. The solitons localized "shoulders" on both sides of the density depletion.

Numerical solutions of the time-dependent system of Eqs. (60) and (61) is displayed in Fig. 3. with initial conditions close (but not equal) to the ones in Fig. 2. Two very clear and long-lived dark solitons are visible, associated with a positive potential of $\varphi \approx 3$, in agreement with the quasi-stationary solution of Fig. 2 for $A=5$. In addition there are oscillations and wave turbulence in the time-dependent solution presented in Fig. 3. Hence, the dark solitons seem to be robust structures that can withstand perturbations and turbulence during a considerable time.

For the two-dimensional $(D=2)$ system, it is possible to find vortex structures of the form $\Psi=\psi(r) \exp (i s \theta-i \Omega t)$, where $r$ and $\theta$ are the polar coordinates defined via $x=r \cos (\theta)$ and $y=r \sin (\theta), \Omega$ is a constant frequency shift, and $s=0, \pm 1, \pm 2, \ldots$ for different excited states (charge states). The index $s$ is also known as the circulation number [51]. With this ansatz, Eqs. (60) and $(61)$ can be written in the form

$$
\left[\Omega+A\left(\frac{d^{2}}{d r^{2}}+\frac{1}{r} \frac{d}{d r}-\frac{s^{2}}{r^{2}}\right)+\varphi-|\psi|^{2}\right] \psi=0,
$$

and

$$
\left(\frac{d^{2}}{d r^{2}}+\frac{1}{r} \frac{d}{d r}\right) \varphi=|\psi|^{2}-1
$$



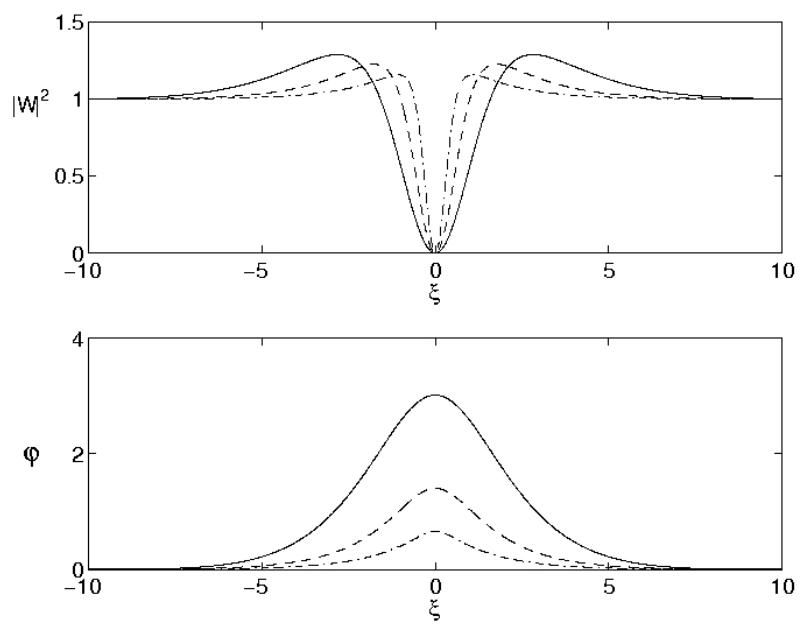

FIG. 2: The electron density $|W|^{2}$ (the upper panel) and electrostatic potential $\varphi$ (the lower panel) associated with a dark soliton supported by the system of equations (67) and (68), for $A=5$ (solid lines), $A=1$ (dashed lines), and $A=0.2$ (dash-dotted line). After Ref. [22].
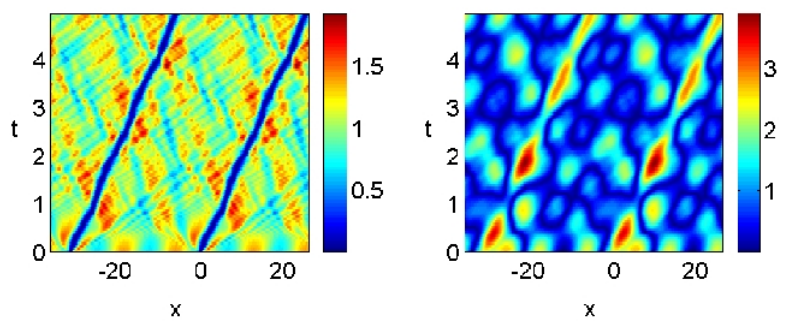

FIG. 3: The time-development of the electron density $|\Psi|^{2}$ (left-hand panel) and electrostatic potential $\varphi$ (the righthand panel), obtained from a simulation of the system of equations 60 and 61 . The initial condition is $\Psi=0.18+$ $\tanh [20 \sin (x / 10)] \exp (i K x)$, with $K=v_{0} / 2 A, A=5$ and $v_{0}=5$. After Ref. [22.

respectively, where the boundary conditions $\psi=1$ and $\varphi=d \psi / d r=0$ at $r=\infty$ determine the constant frequency $\Omega=1$. Different signs of charge state $s$ describe different rotation directions of the vortex. For $s \neq 0$, one must have $\psi=0$ at $r=0$, and from symmetry considerations one has $d \varphi / d r=0$ at $r=0$. Figure 4 shows numerical solutions of Eqs. (70) and 710 for different $s$ and for $A=5$. Here the vortex is characterized by a complete depletion of the electron density at the core of the vortex, and is associated with a positive electrostatic potential.

Figure 5 shows time-dependent solutions of Eqs. (60) and (61) in two space dimensions for singly charged $(s= \pm 1)$ vortices, where, in the initial condition, four vortex-like structures were placed at some distance from each other. The initial conditions were such that the vortices are organized in two vortex pairs, with $s_{1}=+1, s_{2}=-1, s_{3}=-1$, and $s_{4}=+1$, seen in the upper panels of Fig. 5. The vortices in the pairs have opposite polarity on the electron fluid rotation, as seen in the in the upper right panel of Fig. 5. Interestingly, the "partners" in the vortex pairs attract each other and propagate together with a constant velocity, and in the collision and interaction of the vortex pairs (see the second and third pairs of panels in Fig. 5), the vortices keep their identities and change partners, resulting into two new vortex pairs which propagate obliquely to the original propagation direction. On the other hand, as shown in Fig. 6. vortices that are multiply charged $\left(\left|s_{j}\right|>1\right)$ are unstable. Here the system of Eqs. (60) and (61) was again solved numerically with the same initial condition as the one in Fig. 5. but with doubly charged vortices $s_{1}=+2$, $s_{2}=-2, s_{3}=-2$, and $s_{4}=+2$. The second row of panels in Fig. 6 reveals that the vortex pairs keep their identities for some time, while a quasi one-dimensional density cavity is formed between the two vortex pairs. At a later stage, the four vortices dissolve into complicated nonlinear structures and wave turbulence. Hence, the nonlinear dynamics is very different between singly and multiply charged solitons, where only singly charged vortices are long-lived and keep their identities. This is in line with previous results on the nonlinear Schrödinger equation, where it was noted that vortices with higher charge states are unstable [149. 

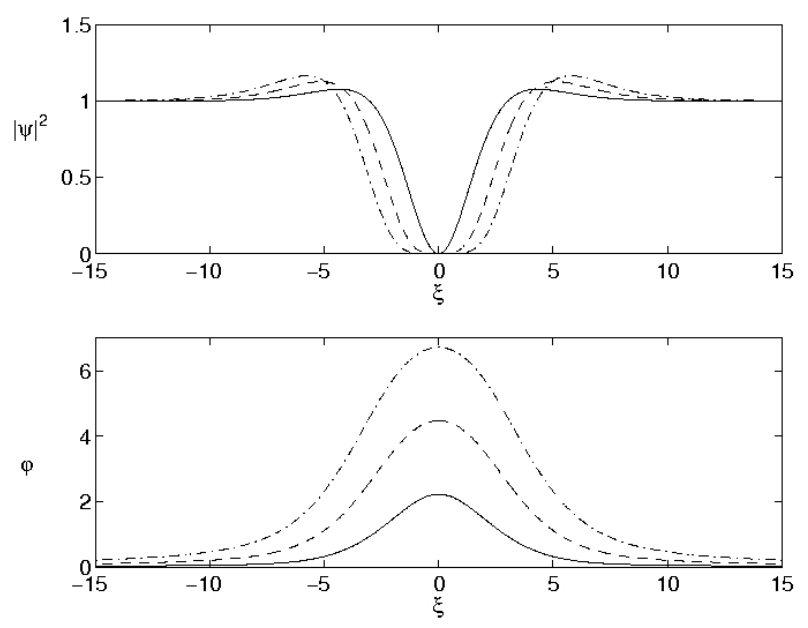

FIG. 4: The electron density $|\Psi|^{2}$ (upper panel) and electrostatic potential $\varphi$ (lower panel) associated with a two-dimensional vortex supported by the system (70) and (71), for the charge states $s=1$ (solid lines), $s=2$ (dashed lines) and $s=3$ (dash-dotted lines). We used $A=5$ in all cases. After Ref. [22].

\section{B. Localized ion wave excitations in quantum plasmas}

In his classic paper, Haas et al. 61 developed both small and large amplitude theories for one-dimensional solitary ion waves in a dense quantum plasmas. They found that the dynamics of small amplitude solitary waves is governed by the Kortweg de-Vries (k-dV) equation

$$
\frac{\partial U}{\partial \tau}+2 U \frac{\partial U}{\partial \xi}+\frac{1}{2}\left(1-\frac{H}{8}\right) \frac{\partial^{3} U}{\partial \xi^{3}}=0
$$

where $U$ represents the relative (with respect to $n_{0}$ ) ion density perturbation, the time and space variables are in units of the ion plasma period $\omega_{p i}^{-1}$ and the electron Thomas-Fermi radius $\left(k_{B} T_{F e} / 4 \pi n_{0} e^{2}\right)^{1 / 2}$, respectively.

The $\mathrm{K}-\mathrm{dV}$ equation admits both the solitary and the periodic (cnoidal) waves [150. Introducing the wave form $U\left(\eta=\xi-M_{s} \tau\right)$, we can write (72) in the stationary frame as

$$
\frac{1}{2}\left(1-\frac{H}{8}\right) \frac{\partial^{2} U}{\partial \eta^{2}}-M_{s} U+U^{2}+C=0,
$$

where $M_{s}$ represents the Mach number, and $C$ is a constant of integration. In the special case when $U$ and its derivatives tend to zero at $\pm \infty, C=0$. Multiplying (73) by $\partial U / \partial \eta$ one can integrate once the resultant equation, and express it in the form of an energy integral [151, 152, 153]. The resulting solitary wave solution of (73) is

$$
U=U_{m} \operatorname{sech}^{2}\left(\eta / \eta_{0}\right),
$$

where $U_{m}=\left(3 M_{s} / 2\right)$ and $\eta_{0}=\left(2 / M_{s}\right)^{1 / 2}(1-H / 8)^{1 / 2}$ are the maximum amplitude and the width of the soliton. We see that compressive solitary wave solutions are possible if $0<H<8$.

There also exist possibility of one-dimensional large amplitude localized ion wave excitations. To demonstrate this, we assume that the quantum force acting on the electrons dominates over the quantum statistical pressure, viz. $k_{B} T_{F e} n_{e} \ll\left(\hbar^{2} / 4 m_{e}\right) \partial^{2} n_{e} / \partial x^{2}$. Hence, the electron density is obtained from 62

$$
e \phi+\frac{\hbar^{2}}{2 m_{e} \sqrt{n_{e}}} \frac{\partial^{2} \sqrt{n_{e}}}{\partial x^{2}}=0 .
$$

The electrons are coupled with ions through the space charge electric field $(-\nabla \phi)$.

The dynamics of singly charged ions is governed by the ion continuity

$$
\frac{\partial n_{i}}{\partial t}+\frac{\partial\left(n_{i} u_{i}\right)}{\partial x}=0
$$



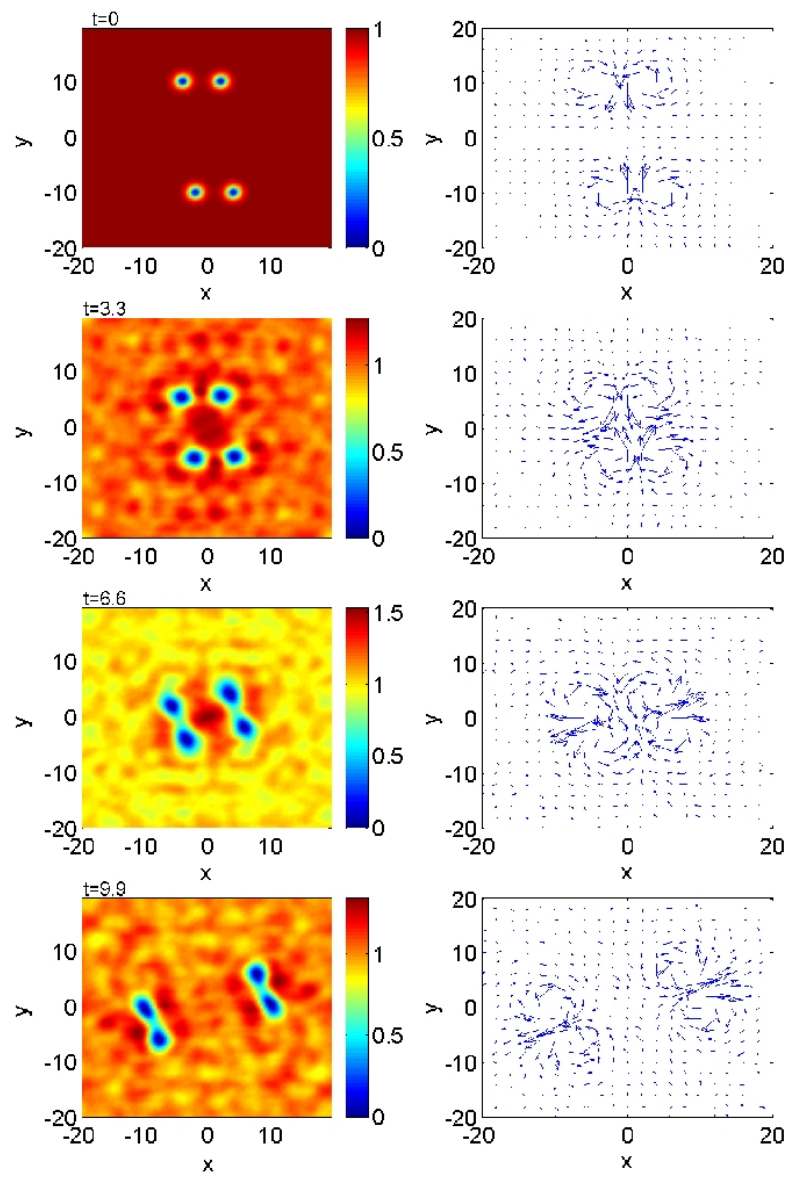

FIG. 5: The electron density $|\Psi|^{2}$ (left panel) and an arrow plot of the electron current $i\left(\Psi \nabla \Psi^{*}-\Psi^{*} \nabla \Psi\right)$ (right panel) associated with singly charged $(|s|=1)$ two-dimensional vortices, obtained from a simulation of the time-dependent system of equations 60 and (61), at times $t=0, t=3.3, t=6.6$ and $t=9.9$ (upper to lower panels). We used $A=5$. The singly charged vortices form pairs and keep their identities. After Ref. 22.

and ion momentum equation

$$
m_{i}\left(\frac{\partial}{\partial t}+u_{i} \frac{\partial}{\partial x}\right) u_{i}=-e \frac{\partial \phi}{\partial x}
$$

where $u_{i}$ is $x$ component of the ion fluid velocity perturbation. The system of Eqs. 75- 77 is closed by the Poisson equation

$$
\frac{\partial^{2} \phi}{\partial x^{2}}=4 \pi e\left(n_{e}-n_{i}\right) .
$$

We now look for stationary nonlinear ion wave structures moving with a constant speed $u_{0}$. Hence, all unknowns depend only on the variable $\xi=x-u_{0} t$. Defining $\sqrt{n_{e}} \equiv \psi$, Eq. 75 takes the form

$$
\frac{\hbar^{2}}{2 m_{e}} \frac{\partial^{2} \psi}{\partial \xi^{2}}+e \phi \psi=0 .
$$

Equations (76) and (77) can be integrated once with the boundary conditions $n_{i}=n_{0}$ and $u_{i}=0$ at $\xi=|\infty|$, and the results can be combined to have

$$
n_{i}=\frac{n_{0} u_{0}}{\sqrt{u_{0}^{2}-2 e \phi / m_{i}}} .
$$



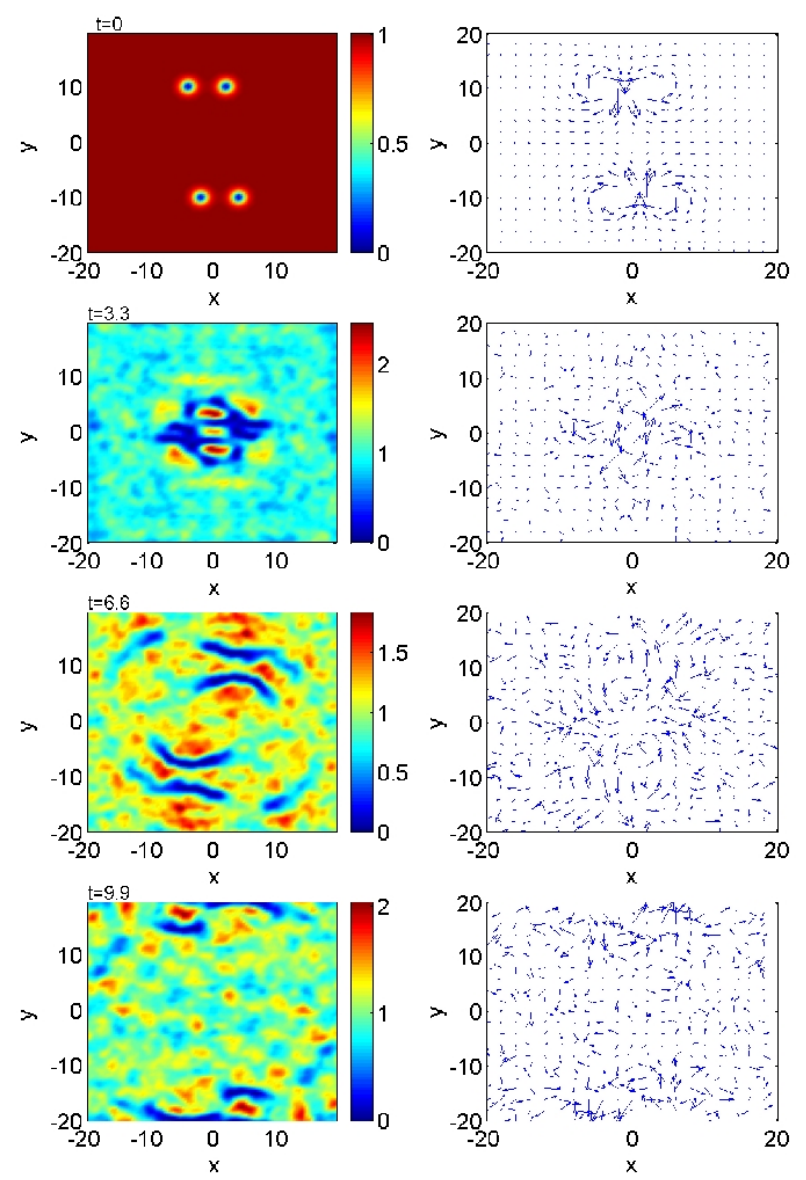

FIG. 6: The electron density $|\Psi|^{2}$ (left panel) and an arrow plot of the electron current $i\left(\Psi \nabla \Psi^{*}-\Psi^{*} \nabla \Psi\right)$ (right panel) associated with double charged $(|s|=2)$ two-dimensional vortices, obtained from a simulation of the time-dependent system of Eqs. 60 and (61), at times $t=0, t=3.3, t=6.6$ and $t=9.9$ (upper to lower panels). We used $A=5$. The doubly charged vortices dissolve into nonlinear structures and wave turbulence. After Ref. 22.

Inserting 80 into 78 we obtain

$$
\frac{\partial^{2} \phi}{\partial \xi^{2}}=4 \pi e\left(\psi^{2}-\frac{n_{0} u_{0}}{\sqrt{u_{0}^{2}-2 e \phi / m_{i}}}\right) .
$$

Equations 79 and (81) are the desired equations for studying the nonlinear ion waves in dense quantum plasmas.

It is convenient to introduce dimensionless quantities [see below Eq. 83] ] into Eqs. (79) and [81), and rewrite them as

$$
\frac{\partial^{2} \Psi}{\partial X^{2}}+\frac{\Phi \Psi}{2}=0
$$

and

$$
\frac{\partial^{2} \Phi}{\partial X^{2}}-\Psi^{2}+\frac{M}{\sqrt{M-2 \Phi}}=0,
$$

where we have normalized the space variable as $X=k_{q} \xi$, the electron wave function as $\Psi=\sqrt{n_{0}} \psi$, and the potential as $\Phi=e \phi / m_{i} c_{q}^{2}$. Here $c_{q}=\omega_{p i} / k_{q}$ is the quantum ion wave speed and $k_{q}=\left(2 m_{e} \omega_{p e} / \hbar\right)^{1 / 2}$ is the quantum wavenumber. The quantum "Mach number" is defined as $M=u_{0} / c_{q}$.

We note that the coupled Eqs. 82 and 83 admit a conserved quantity

$$
\mathcal{H}=-2\left(\frac{\partial \Psi}{\partial X}\right)^{2}+\frac{1}{2}\left(\frac{\partial \Phi}{\partial X}\right)^{2}-\Phi \Psi^{2}-M\left(\sqrt{M^{2}-2 \Phi}-M\right)=0,
$$


where we have used the boundary conditions $\Phi=\partial \Phi / \partial X=\partial \Psi / \partial X=0$, and $\Psi=1$ at $|X|=\infty$. For a symmetric solitary ion wave structure, we can assume that $\Phi=\Phi_{\max }$ and $\Psi=\Psi_{\max }$, as well as $\partial \Psi / \partial X=\partial \Phi / \partial X=0$ at $X=0$. Hence, at $X=0$ Eq. (84) yields

$$
\Phi_{\max } \Psi_{\max }^{2}+M\left(\sqrt{M^{2}-2 \Phi_{\max }}-M\right)=0 .
$$

In the wave-breaking limit, where $M=\left(2 \Phi_{\max }\right)^{1 / 2}$, we find that $-\Phi_{\max } \Psi_{\max }^{2}+2 \Phi_{\max }=0$, or $\Psi_{\max }=2$. Accordingly, the electron density will locally rise to twice the background density at wave breaking.
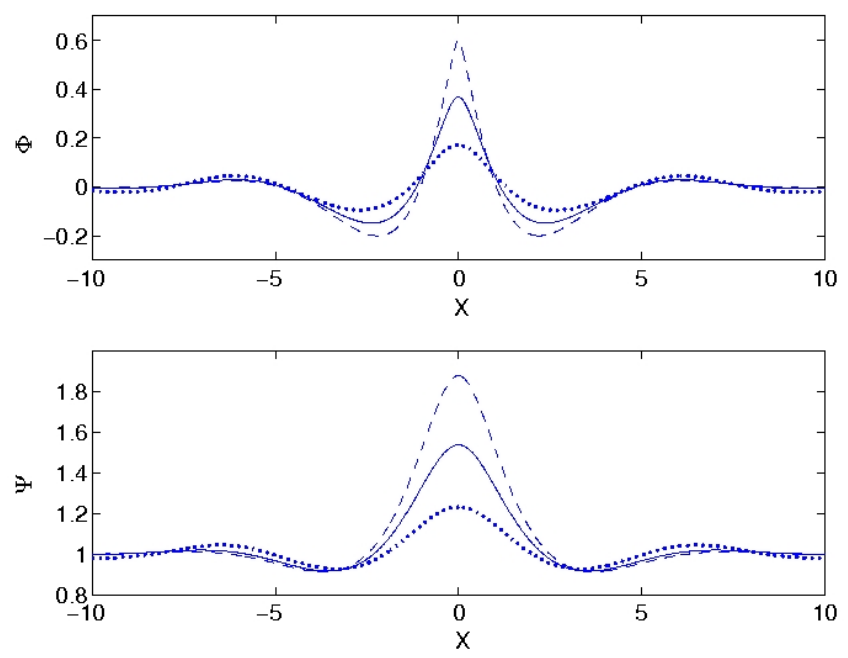

FIG. 7: The profiles of the potential $\Phi$ (top panel) and the electron density $\Psi^{2}$ (bottom panel) as a function of $X$, for different values of the Mach number: $M=1.1$ (dashed curves), $M=0.9$ (solid curve), and $M=0.75$ (dotted curve). After Ref. 62 .

In Fig. 7. numerical solutions of Eqs. (82) and (83) are displayed, showing profiles of the electrostatic potential and electron number densities for different values of $M$. We see that both the electrostatic potential and electron density have localized and strongly peaked maxima and an oscillatory tail. The latter is in sharp contrast to the classical (non-quantum) case, where the ion acoustic solitary waves have a monotonic profile, which in the small amplitude limit, where the system is governed by the Korteweg-de Vries equation, assumes a secant hyperbolicus shape. We observe from Fig. 7 that $M=1.1$ is close to the wave breaking limit above which there do not exist solitary wave solutions. Our numerical investigation also suggests that there is a lower limit of $M$ (slightly lower than 0.75), below which the solitary wave solution vanishes.

\section{QUANTUM FLUID TURBULENCE}

The statistical properties of turbulence and its associated electron transport at nanoscales in quantum plasmas has been investigated in both 2D and 3D by means of the coupled NLS and Poisson equations [66, 67. It has been found that the nonlinear coupling between the EPOs of different scale sizes gives rise to small-scale electron density structures, while the electrostatic potential cascades towards large-scales. The total energy associated with the quantum electron plasma turbulence, nonetheless, processes a characteristic, non-Kolmogorov-like spectrum. The electron diffusion caused by the electron fluid turbulence is consistent with the dynamical evolution of turbulent mode structures.

To investigate the quantum electron fluid turbulence in 3D, we use the nonlinear Schrödinger-Poisson equations [21, 22, 67 ,

$$
i \sqrt{2 H} \frac{\partial \Psi}{\partial t}+H \nabla^{2} \Psi+\varphi \Psi-|\Psi|^{4 / 3} \Psi=0
$$

and

$$
\nabla^{2} \varphi=|\Psi|^{2}-1
$$

which govern the dynamics of nonlinearly interacting EPOs of different wavelengths. In Eqs. (86) and (87) the wave function is normalized by $\sqrt{n_{0}}$, the electrostatic potential by $k_{B} T_{F e} / e$, the time $t$ by the electron plasma period $\omega_{p e}^{-1}$, and the space $\mathbf{r}$ by the Thomas-Fermi Debye radius $V_{F e} / \omega_{p e}$. We have introduced the notation $\sqrt{H}=\hbar \omega_{p e} / \sqrt{2} k_{B} T_{F e}$. 
The nonlinear mode coupling interaction studies are performed to investigate the multi-scale evolution of a decaying $3 \mathrm{D}$ electron fluid turbulence, which is described by Eqs. 86 and (87). All the fluctuations are initialized isotropically (no mean fields are assumed) with random phases and amplitudes in Fourier space, and evolved further by the integration of Eqs. (86) and (87), using a fully de-aliased pseudospectral numerical scheme [154] based on the Fourier spectral methods. The spatial discretization in our 3D simulations uses a discrete Fourier representation of turbulent fluctuations. The numerical algorithm employed here conserves energy in terms of the dynamical fluid variables and not due to a separate energy equation written in a conservative form. The evolution variables use periodic boundary conditions. The initial isotropic turbulent spectrum was chosen close to $k^{-2}$, with random phases in all three directions. The choice of such (or even a flatter than -2) spectrum treats the turbulent fluctuations on an equal footing and avoids any influence on the dynamical evolution that may be due to the initial spectral non-symmetry. The equations are advanced in time using a second-order predictor-corrector scheme. The code is made stable by a proper de-aliasing of spurious Fourier modes, and by choosing a relatively small time step in the simulations. Our code is massively parallelized using Message Passing Interface (MPI) libraries to facilitate higher resolution in a 3D computational box, with a resolution of $128^{3}$ grid points.

We study the properties of 3D fluid turbulence, composed of nonlinearly interacting EPOs, for two specific physical systems. These are the dense plasmas in the next generation laser-based plasma compression (LBPC) schemes [81] as well as in superdense astrophysical objects 96, 97, 98, (e.g. white dwarfs). It is expected that in LBPC schemes, the electron number density may reach $10^{27} \mathrm{~cm}^{-3}$ and beyond. Hence, we have $\omega_{p e}=1.76 \times 10^{18} \mathrm{~s}^{-1}, T_{F}=1.7 \times 10^{-9} \mathrm{erg}$, $\hbar \omega_{p e}=1.7 \times 10^{-9} \mathrm{erg}$, and $H=1$. The Fermi Debye length $\lambda_{D}=0.1 \AA$. On the other hand, in the interior of white dwarfs, we typically have $n_{0} \sim 10^{30} \mathrm{~cm}^{-3}$, yielding $\omega_{p e}=5.64 \times 10^{19} \mathrm{~s}^{-1}, T_{F}=1.7 \times 10^{-7} \mathrm{erg}, \hbar \omega_{p e}=5.64 \times 10^{-8} \mathrm{erg}$, $H \approx 0.3$, and $\lambda_{D}=0.025 \AA$. The numerical solutions of Eqs. (86) and (87) for $H=1$ and $H=0.025$ (corresponding to $n_{0}=10^{27} \mathrm{~cm}^{-3}$ and $n_{0}=10^{30} \mathrm{~cm}^{-3}$, respectively) are displayed in Figs. 8 and 9 , respectively, which are the electron number density and electrostatic (ES) potential distributions in the $(x, y)$-plane.
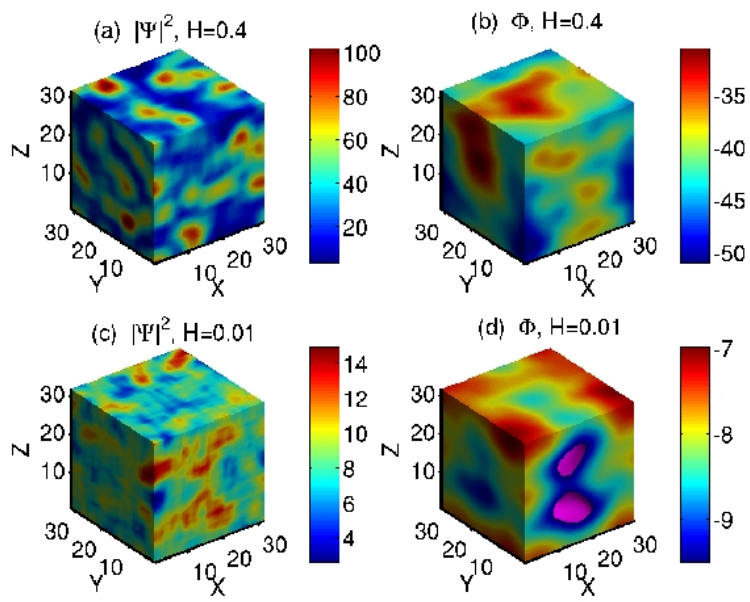

FIG. 8: Small scale fluctuations in the electron density resulted from a steady turbulence simulations of our 3D electron plasma, for $H=0.4$ (top panels) and $H=0.01$ (bottom panels). Forward cascades are responsible for the generation of small-scale fluctuations seen in panels (a) and (c). Large scale structures are present in the electrostatic potential, seen in panels (b) and (d), essentially resulting from an inverse cascade. After Ref. [67.

Figures 8 and 9 reveal that the electron density distribution has a tendency to generate smaller length-scale structures, while the ES potential cascades towards larger scales. The co-existence of the small and larger scale structures in turbulence is a ubiquitous feature of various 3D turbulence systems. For example, in 3D hydrodynamic turbulence, the incompressible fluid admits two invariants, namely the energy and the mean squared vorticity. The two invariants, under the action of an external forcing, cascade simultaneously in turbulence, thereby leading to a dual cascade phenomena. In these processes, the energy cascades towards longer length-scales, while the fluid vorticity transfers spectral power towards shorter length-scales. Usually, a dual cascade is observed in a driven turbulence simulation, in which certain modes are excited externally through random turbulent forces in spectral space. The randomly excited Fourier modes transfer the spectral energy by conserving the constants of motion in $k$-space. On the other hand, in freely decaying turbulence, the energy contained in the large-scale eddies is transferred to the smaller scales, leading to a statistically stationary inertial regime associated with the forward cascades of one of the invariants. Decaying turbulence often leads to the formation of coherent structures as turbulence relaxes, thus making the nonlinear interactions rather inefficient when they are saturated. The power spectrum exhibits an interesting feature in our 3D 


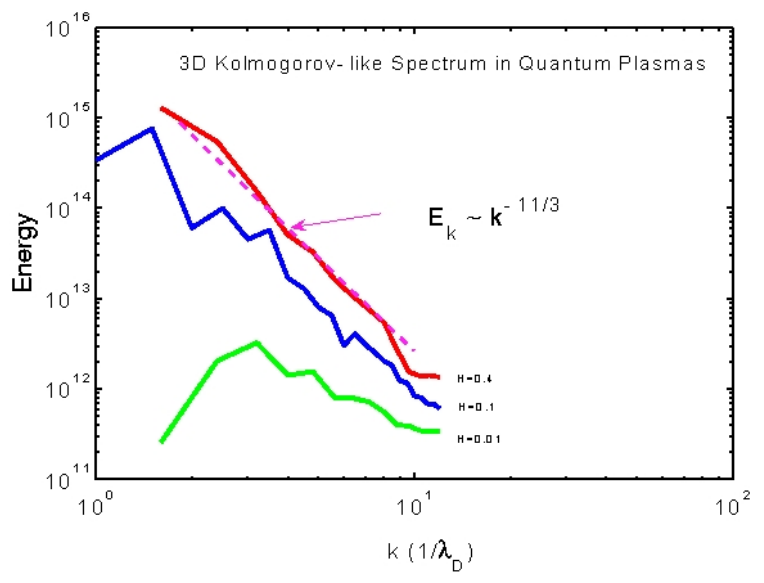

FIG. 9: Power spectrum of 3D EPOs in the forward cascade regime. A Kolmogorov-like spectrum $\sim k^{-11 / 3}$ is observed for $H=0.4$. The spectral index changes as a function of $H$. The numerical resolution is $128^{3}$. After Ref. 67.

electron plasma system, unlike the 3D hydrodynamic turbulence [155, 156, 157, 158. The spectral slope in the 3D quantum electron fluid turbulence is close to the Iroshnikov-Kraichnan power law [159, 160 $k^{-3 / 2}$, rather than the usual Kolomogrov power law [155] $k^{-5 / 3}$. We further find that this scaling is not universal and is determined critically by the quantum tunneling effect. For instance, for a higher value of $\mathrm{H}=1.0$ the spectrum becomes more flat (see Fig 9). Physically, the flatness (or deviation from the $k^{-5 / 3}$ ), results from the short wavelength part of the EPOs spectrum which is controlled by the quantum tunneling effect associated with the Bohm potential. The peak in the energy spectrum can be attributed to the higher turbulent power residing in the EPO potential, which eventually leads to the generation of larger scale structures, as the total energy encompasses both the electrostatic potential and electron density components. In our dual cascade process, there is a delicate competition between the EPO dispersions caused by the statistical pressure law (giving the $k^{2} V_{F}^{2}$ term, which dominates at longer scales) and the quantum Bohm potential (giving the $\hbar^{2} k^{4} / 4 m_{e}^{2}$ term, which dominates at shorter scales with respect to a source). Furthermore, it is interesting to note that exponents other than $k^{-5 / 3}$ have also been observed in numerical simulations [161, 162] of the Charney and 3D incompressible Navier-Stokes equations. Recently, Paoletti et al 163 have examined the velocity statistics of quantum turbulence in superfluid ${ }^{4} \mathrm{He}$, and found that it significantly differs from classical turbulence due to the topological interactions of vortices that are different from those in classical fluids.

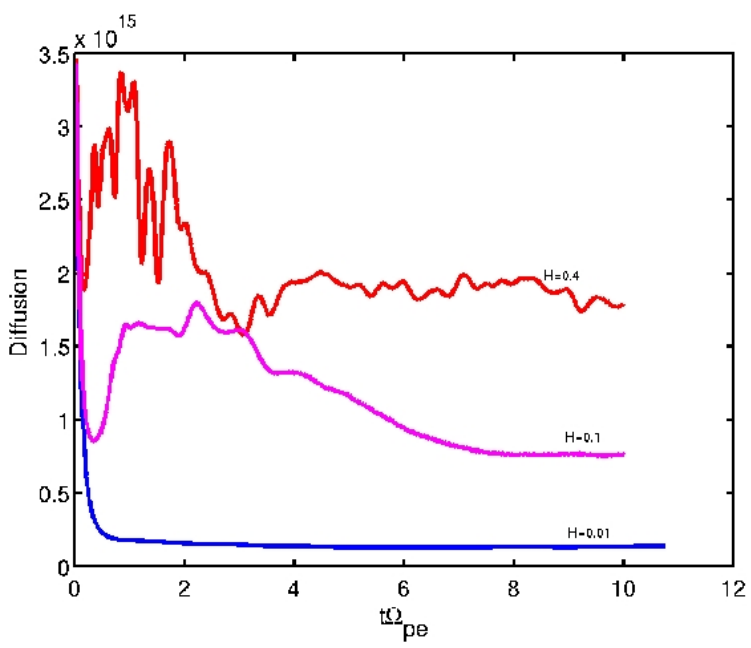

FIG. 10: Time evolution of an effective electron diffusion coefficient associated with the large-scale electrostatic potential and the small-scale electron density, for $H=0.4, H=0.1$ and $H=0.01$. Smaller values of $H$ corresponds to a small effective diffusion coefficient, which characterizes the presence of small-scale turbulent eddies that suppress the electron transport. After Ref. 67]. 
We finally estimate the electron diffusion coefficient in the presence of small and large scale turbulent EPOs in our quantum plasma. An effective electron diffusion coefficient caused by the momentum transfer can be calculated from $D_{\text {eff }}=\int_{0}^{\infty}\left\langle\mathbf{P}(\mathbf{r}, t) \cdot \mathbf{P}\left(\mathbf{r}, t+t^{\prime}\right)\right\rangle d t^{\prime}$, where $\mathbf{P}$ is electron momentum and the angular bracket denotes spatial averages and the ensemble averages are normalized to unit mass. The effective electron diffusion coefficient, $D_{e f f}$, essentially relates the diffusion processes associated with random translational motions of the electrons in nonlinear plasmonic fields. We compute $D_{\text {eff }}$ in our simulations, to measure the turbulent electron transport that is associated with the turbulent structures that we have reported herein. It is observed that the effective electron diffusion is lower when the field perturbations are Gaussian. On the other hand, the electron diffusion increases rapidly with the eventual formation of longer length-scale structures, as shown in Fig. 10. The electron diffusion due to large scale potential distributions in quantum plasmas dominates substantially, as depicted by the solid-curve in Fig. 10. Furthermore, in the steady-state, nonlinearly coupled EPOs form stationary structures, and $D_{\text {eff }}$ saturates eventually. Thus, remarkably an enhanced electron diffusion results primarily due to the emergence of large-scale potential structures in our 3D quantum plasma.

\section{KINETIC PHASE-SPACE STRUCTURES}

In the preceding sections, we have discussed the properties of quantized coherent structures and 3D quantum electron fluid turbulence based on the coupled Schrödinger and Poisson equations. Thus, it has been assumed that nonlinearly interacting plasma waves are spontaneously created by some known physical processes (e.g. the beamplasma instability) in quantum plasmas.

The formation of electrostatic kinetic phase space structures, based on the Vlasov-Poisson equations, in classical plasmas has been well documented [164. In the following, we shall discuss quasilinear aspects [165] of the EPOs that are governed by the Wigner-Poisson system (i.e. a quantum analogue of the Vlasov-Poisson system). Specifically, we focus on kinetic phase-space nonlinear structures arising from the trapping of electrons in the finite amplitude wave potential and the self-consistent modification of the electron distribution function in the presence of nano-kinetic structures.

To study the differences in the nonlinear evolution of the Wigner and Vlasov equations, we have simulated the wellknown bump-on-tail instability [165, whereby a high-velocity beam is used to destabilize a Maxwellian equilibrium. We use the initial condition $f=(1+\delta)\left(n_{0} / \sqrt{2 \pi} v_{t h}\right)\left[0.8 \exp \left(-v^{2} / 2 v_{t h}^{2}\right)+0.4 \exp \left(-2\left(v-2.5 v_{t h}\right)^{2} / v_{t h}^{2}\right)\right]$, where $\delta$ represents random fluctuations of order $10^{-5}$ that help seed the instability (see Fig. 1). Here $v_{t h}=\sqrt{k_{B} T_{e} / m_{e}}$ is the electron thermal speed. We use periodic boundary conditions with spatial period $L=40 \pi \lambda_{D e}$, where $\lambda_{D e}=v_{t h} / \omega_{p}$ is the Debye length. Three simulations were performed, with different values of the normalized Planck constant, defined as $H=\hbar \omega_{p e} / m v_{t h}^{2}: H=0$ (Vlasov), $H=1$, and $H=2$.

In order to highlight the transient oscillations in velocity space, we first perturb the above equilibrium with a monochromatic wave having $k \lambda_{D e}=0.25$ (i.e., a wavelength of $8 \pi \lambda_{D e}$ ). Figure 11 shows the results from simulations of the Wigner-Poisson and Vlasov-Poisson systems. In both simulations, due to the bump-on-tail instability, electrostatic waves develop nonlinearly and create periodic trapped-particle islands (electron holes) with the wavenumber $k=$ $0.25 \lambda_{D e}^{-1}$. The theory predicts the formation of velocity-space oscillations in the Wigner evolution, which should be absent in the classical (Vlasov) simulations. This is the case in the results presented in Fig. 2, where the oscillations are clearly visible.

When the initial excitation is broad-band (i.e., wavenumbers $0.05 \leq k \lambda_{D e} \leq 0.5$ are excited), the electron holes start merging together at later times due to the sideband instability [166, 167] (see Fig. 12). At this stage, mode coupling becomes important and quasilinear theory is not capable of describing these effects. As the system evolves toward larger spatial wavelength, the evolution becomes progressively more classical, with the appearance of a plateau in the resonant region. Nevertheless, at $\omega_{p e} t=500$ the Wigner solution still displays some oscillatory behavior in velocity space, which is absent in the Vlasov evolution.

From the experimental viewpoint, recent collective x-ray scattering observations in warm dense matter [118] revealed a measurable shift in the plasmon frequency due to quantum effects. In the nonlinear regime (strong excitations), this effect could lead to trapping of electrons in the wave potential of the plasmons, and the subsequent formation of the kind of phase space structures discussed here. Furthermore, we note that there also exists a theoretical description [168] of quantum corrected electron holes based on the perturbative treatment of the Wigner-Poisson equations.

\section{MAGNETIC FIELDS IN QUANTUM PLASMAS}

There are several mechanisms by which magnetic fields in classical plasmas can be generated. They include i) non-parallel density and temperature gradients (the so called Biermann battery [169]), ii) the electron temperature 

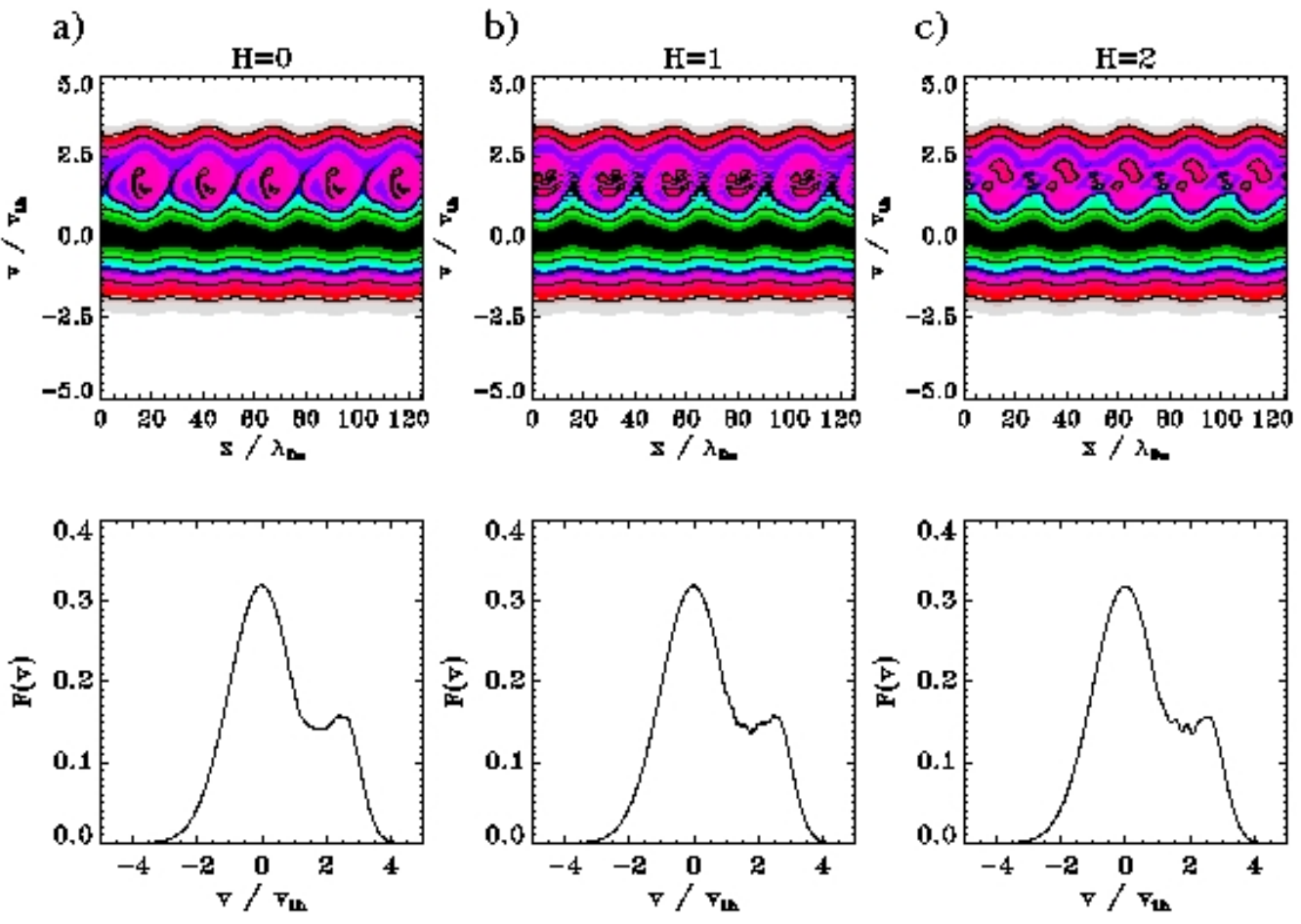

FIG. 11: (Color online) Simulations of the Wigner-Poisson and Vlasov-Poisson systems, at time $\omega_{p e} t=200$, for a) $H=0$ (Vlasov), b) $H=1$ (Wigner), and c) $H=2$ (Wigner). Initially monochromatic spectrum. Top panels: electron distribution function $f(x, v)$ in phase space. Bottom panels: spatially averaged electron distribution function $F(v)$ in velocity space. After Ref. [165.

anisotropy (known as the Weibel instability [170), iii) counterstreaming electron beams [171, 172, and iv) the ponderomotive forces of laser beams [173, 174, 175, 176, 177, [178, 179].

In the following, we discuss two possibilities for the magnetic field generation in quantum plasmas.

\section{A. The quantum Weibel instability}

We first discuss linear and nonlinear aspects $180,181,182,183$ of the Weibel instability that is driven by equilibrium Fermi-Dirac electron temperature anisotropic distribution function in a nonrelativistic dense quantum plasma. It is well known [184 that a dense quantum plasma with an isotropic equilibrium distribution function does not admit any purely growing linear modes. This can be verified, for instance, from the expression for the imaginary part of the transverse dielectric function, as derived by Lindhard [185], for a fully degenerate non-relativistic Fermi plasma. It can be proven (see Eq. (30) of [186]) that the only exception would be for extremely small wavelengths, so that $k>2 k_{F}$, where $k_{F}$ the characteristic Fermi wave number of the system. However, in this situation the wave would be super-luminal. On the other hand, in a classical Vlasov-Maxwell plasma containing anisotropic electron distribution function, we have a purely growing Weibel instability [170, via which dc magnetic fields are created. The electron temperature anisotropy may arises due to the heating of the plasma by laser beams [187, where there is a signature of the Weibel instability as well. In the next generation intense laser-solid density plasma experiments, it is likely that electrons would be degenerate and that electron temperature anisotropy may develop due to an anisotropic electron heating by intense laser beams via resonant absorption, similar to the classical laser-plasma-interaction case [188.

Consider linear transverse waves in a dense quantum plasma composed of the electrons and immobile ions, with $\mathbf{k} \cdot \mathbf{E}=0$, where $\mathbf{k}$ is the wave vector and $\mathbf{E}$ is the wave electric field. Following the standard procedure, one then obtains the general dispersion relation [1, 182, 183] for the transverse waves of the Wigner-Maxwell system

$$
\omega^{2}-\omega_{p e}^{2}-k^{2} c^{2}+\frac{m_{e} \omega_{p e}^{2}}{2 n_{0} \hbar} \int d \mathbf{v}\left(\frac{v_{x}^{2}+v_{y}^{2}}{\omega-k v_{z}}\right)\left[f_{0}\left(v_{x}, v_{y}, v_{z}+\frac{\hbar k}{2 m}\right)-f_{0}\left(v_{x}, v_{y}, v_{z}-\frac{\hbar k}{2 m_{e}}\right)\right]=0,
$$

where $\mathbf{v}=\left(v_{x}, v_{y}, v_{z}\right)$ is the velocity vector, and $f_{0}\left(v_{x}, v_{y}, v_{z}\right)$ is the equilibrium Wigner function associated to Fermi systems. For spin 1/2 particles, the equilibrium pseudo distribution function is in the form of a Fermi-Dirac function. 

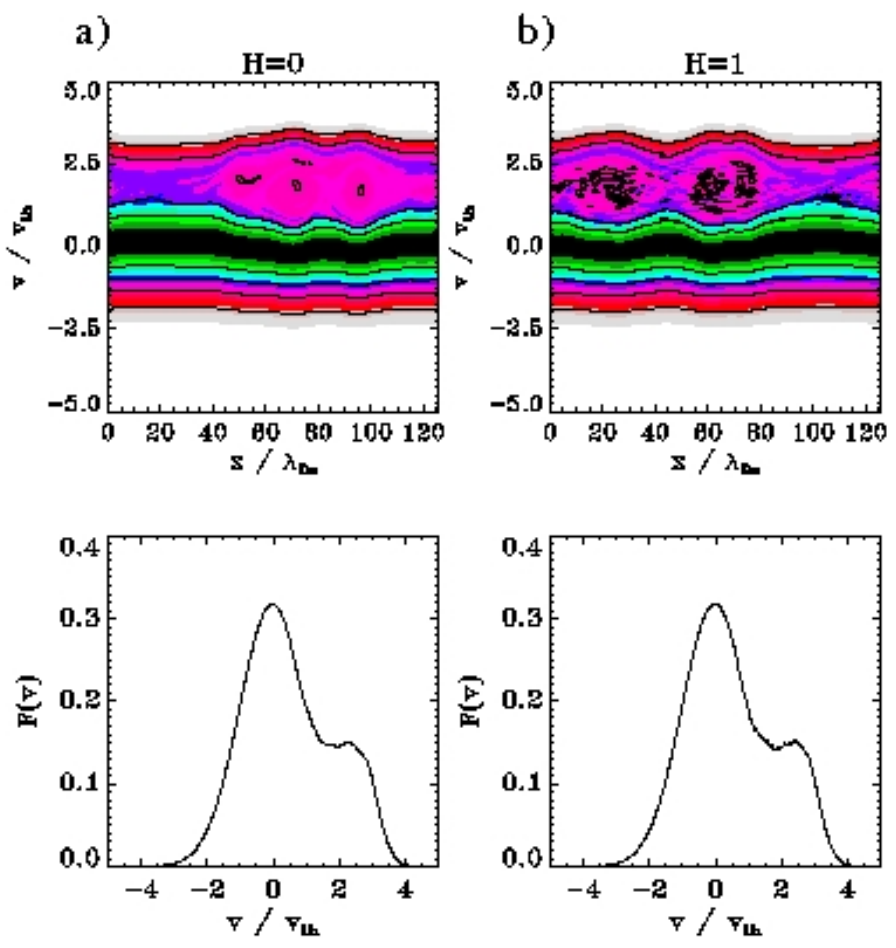

FIG. 12: (Color online) Simulations of the Wigner-Poisson and Vlasov-Poisson systems, for $\omega_{p e} t=500$, a) for $H=0$ (Vlasov) and b) $H=1$ (Wigner). Initially broad wavenumber spectrum. Top panels: electron distribution function $f(x, v)$ in phase space. Bottom panels: spatially averaged electron distribution function $F(v)$ in velocity space. After Ref. [165].

Here we allow for velocity anisotropy and express

$$
f_{0}=\frac{\alpha}{\exp \left[\frac{m}{2}\left(\frac{v_{x}^{2}+v_{y}^{2}}{\kappa_{B} T_{\perp}}+\frac{v_{z}^{2}}{\kappa_{B} T_{\|}}\right)-\beta \mu\right]+1},
$$

where $\mu$ is the chemical potential, and the normalization constant is

$$
\alpha=-\frac{n_{0}}{\operatorname{Li}_{3 / 2}\left(-e^{\beta \mu}\right)}\left(\frac{m_{e} \beta}{2 \pi}\right)^{3 / 2}=2\left(\frac{m_{e}}{2 \pi \hbar}\right)^{3} .
$$

Here $\mathrm{Li}_{3 / 2}$ is a polylogarithm function [189, 190. Also, $\beta=1 /\left[\kappa_{B}\left(T_{\perp}^{2} T_{\|}\right)^{1 / 3}\right]$, where $T_{\perp}$ and $T_{\|}$are related to velocity dispersion in the direction perpendicular and parallel to $z$ axis, respectively. In the special case when $T_{\perp}=T_{\|}$, the usual Fermi-Dirac equilibrium is recovered. The chemical potential is obtained by solving the normalization condition (90), yielding, in particular, $\mu=E_{F}$ in the limit of zero temperature, where $E_{F}=\left(3 \pi^{2} n_{0}\right)^{2 / 3} \hbar^{2} /\left(2 m_{e}\right)$ is the Fermi energy. Also, the Fermi-Dirac distribution $\hat{f}(\mathbf{k})$, where $\mathbf{k}$ is the appropriated wave vector in momentum space, is related to the equilibrium Wigner function 89 by $\hat{f}(\mathbf{k})=(1 / 2)(2 \pi \hbar / m)^{3} f_{0}(\mathbf{v})$, with the factor 2 coming from spin 191, 192. However, these previous works refer to the cases where there is no temperature anisotropy. Notice that it has been suggested [193] that in laser plasmas the Weibel instability is responsible for further increase of $T_{\|}$with time.

Inserting $(89)$ into $(88)$ and integrating over the perpendicular velocity components, we obtain

$$
\omega^{2}-k^{2} c^{2}-\omega_{p e}^{2}\left(1+\frac{T_{\perp}}{T_{\|}} W_{Q}\right)=0,
$$

where

$$
W_{Q}=\frac{1}{2 \sqrt{\pi} H \operatorname{Li}_{3 / 2}\left(-e^{\beta \mu}\right)} \int \frac{d \nu}{\nu-\xi}\left(\operatorname{Li}_{2}\left\{-\exp \left[-\left(\nu+\frac{H}{2}\right)^{2}+\beta \mu\right]\right\}-\operatorname{Li}_{2}\left\{-\exp \left[-\left(\nu-\frac{H}{2}\right)^{2}+\beta \mu\right]\right\}\right) .
$$

In (92), $\mathrm{Li}_{2}$ is the dilogarithm function [189, 190], $H=\hbar k /\left(m_{e} v_{\|}\right)$is a characteristic parameter representing the quantum diffraction effect, $\xi=\omega /\left(k v_{\|}\right)$, and $\nu=v_{z} / v_{\|}$, with $v_{\|}=\left(2 \kappa_{B} T_{\|} / m_{e}\right)^{1 / 2}$. In the simultaneous limit of a 
small quantum diffraction effect $(H \ll 1)$ and a dilute system $\left(e^{\beta \mu} \ll 1\right)$, it can be shown that $W_{Q} \simeq-1-\xi Z(\xi)$, where $Z$ is the standard plasma dispersion function [194. It is important to notice that either (88) or (91) reproduces the transverse dielectric function calculated from the random phase approximation for a fully degenerate quantum plasma [185], in the case of an isotropic system. The simple way to verify this equivalence is to put $T_{\perp}=T_{\|}$in 88 and then take the limit of zero temperature, so that $f_{0}=3 n_{0} /\left(4 \pi V_{F e}^{3}\right)$ for $|\mathbf{v}|<V_{F e}$, and $f_{0}=0$ otherwise.

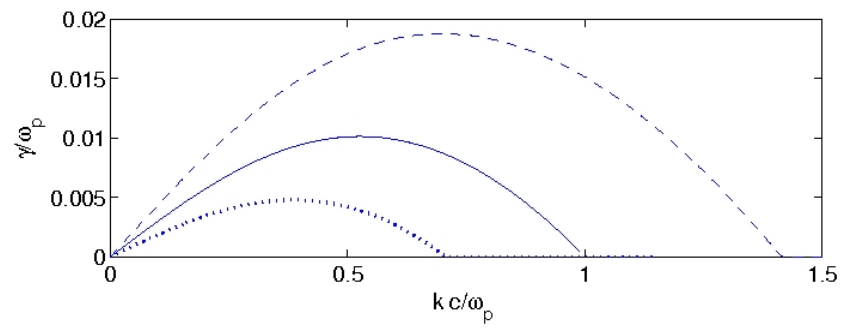

FIG. 13: The growth rate for the Weibel instability of a dense Fermionic plasma with $n_{0}=10^{33} \mathrm{~m}^{-3}\left(\omega_{p e}=1.8 \times 10^{18} \mathrm{~s}^{-1}\right)$ and $\beta \mu=5$, relevant for the next generation inertially compressed material in intense laser-solid density plasma interaction experiments. The temperature anisotropies are $T_{\perp} / T_{\|}=3$ (dashed line), $T_{\perp} / T_{\|}=2$ (solid line) and $T_{\perp} / T_{\|}=1.5($ dotted line), yielding, respectively, $T_{\|}=3.9 \times 10^{6} \mathrm{~K}, T_{\|}=5.2 \times 10^{6} \mathrm{~K}$ and $T_{\|}=6.3 \times 10^{6} \mathrm{~K}$. After Ref. 183 .

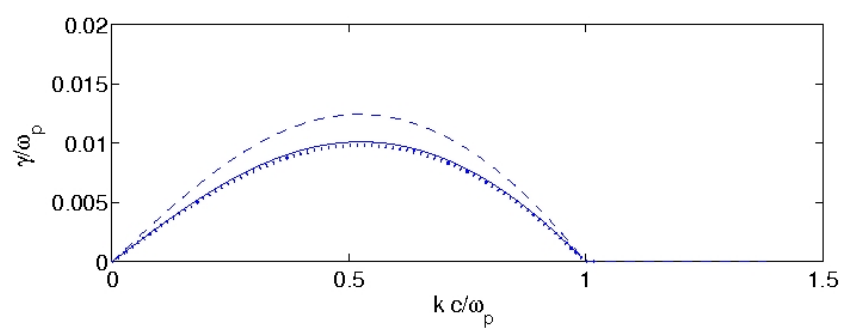

FIG. 14: The growth rate for the Weibel instability of a dense Fermionic plasma with $n_{0}=10^{33} \mathrm{~m}^{-3}\left(\omega_{p e}=1.8 \times 10^{18} \mathrm{~s}^{-1}\right)$. Here the temperature anisotropy is $T_{\perp} / T_{\|}=2$. We used $\beta \mu=1$ (dashed line), $\beta \mu=5$ (solid line) and $\beta \mu=10$ (dotted line), yielding $T_{||}=1.6 \times 10^{7} \mathrm{~K}, T_{\|}=5.2 \times 10^{7} \mathrm{~K}$ and $T_{\|}=2.6 \times 10^{6} \mathrm{~K}$, respectively. After Ref. [183].

We next solve our new dispersion relation (91) for a set of parameters that are representative of the next generation laser-solid density plasma interaction experiments. The normalization condition $(90)$ can also be written as $-\mathrm{Li}_{3 / 2}[-\exp (\beta \mu)]=(4 / 3 \sqrt{\pi})\left(\beta E_{F}\right)^{3 / 2}$, which is formally the same relation holding for isotropic Fermi-Dirac equilibria [8]. For a given value on the product $\beta \mu$ and the density, this relation yields the value $\beta$, from which the temperatures $T_{\perp}$ and $T_{\|}$can be calculated, if we know $T_{\perp} / T_{\|}$. Consider only purely growing modes. From the definition (92), one can show that $W_{Q} \rightarrow-1$ when $\omega=i \gamma \rightarrow 0$ for a finite wavenumber $k$. From (91) we then obtain the maximum wavenumber for instability as $k_{\max }=\left(\omega_{p e} / c\right) \sqrt{T_{\perp} / T_{\|}-1}$. When $T_{\perp} / T_{\|} \rightarrow 1$, the range of unstable wavenumbers shrinks to zero. In Figs. 13 and 14 , we have used the electron number density $n_{0}=10^{33} \mathrm{~m}^{-3}$, which can be obtained in laser-driven compression schemes. The growth rate for different values on $T_{\perp} / T_{\|}$is displayed in Fig. 13. We see that the maximum unstable wavenumber is $k_{\max }=\left(\omega_{p e} / c\right) \sqrt{T_{\perp} / T_{\|}-1}$, as predicted, and that the maximum growth rate occurs at $k \approx k_{\max } / 2$. Figure 13 also reveals that the maximum growth rate of the instability is almost linearly proportional to $T_{\perp} / T_{\|}-1$. In Fig. 14 , we have varied the product $\beta \mu$, which is a measure of the degeneracy of the quantum plasma. We see that for $\beta \mu$ larger than 5 , the instability reaches a limiting value, which is independent of the temperature, while thermal effects start to play an important role for $\beta \mu$ of the order unity.

From several numerical solutions of the linear dispersion relation, it was deduced [183] an approximate scaling law for the instability as $\gamma_{\max } / \omega_{p e}=$ constant $\times n_{0}^{1 / 3}\left(T_{\perp} / T_{\|}-1\right)$, where the constant is approximately $8.5 \times 10^{-14} \mathrm{~s}^{-1} \mathrm{~m}$. Using that $n_{0}=\left(2 m_{e} E_{F} / \hbar^{2}\right)^{3 / 2} /\left(3 \pi^{2}\right) \approx 1.67 \times 10^{36}\left(E_{F} / m_{e} c^{2}\right)^{3 / 2}$, we have

$$
\frac{\gamma_{\max }}{\omega_{p e}}=0.10\left(\frac{E_{F}}{m_{e} c^{2}}\right)^{1 / 2}\left(\frac{T_{\perp}}{T_{\|}}-1\right),
$$

for the maximum growth rate of the Weibel instability in a degenerate Fermi plasma. This scaling law, where the growth rate depends on the Fermi energy and the temperature anisotropy, should be compared to that of a classical plasma [188, 195, where the growth rate depends on the thermal energy and the temperature anisotropy. 

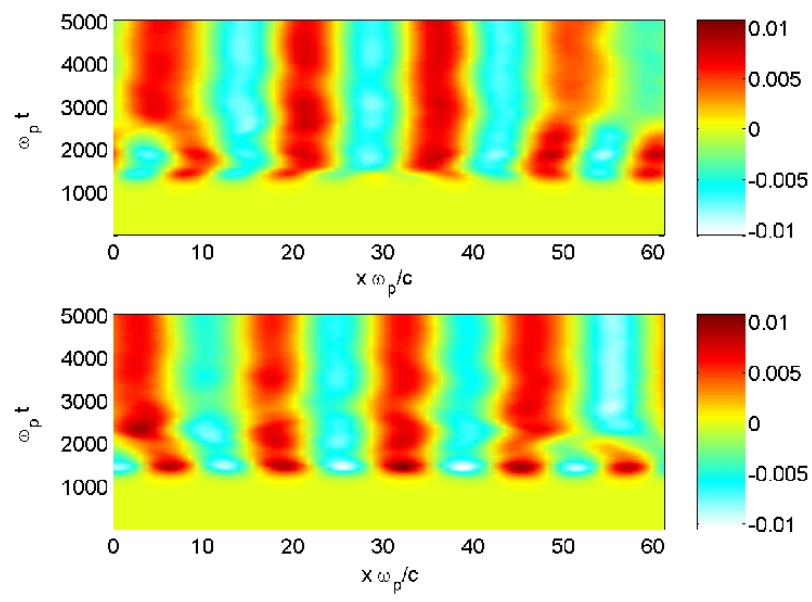

FIG. 15: The magnetic field components $B_{y}$ (top panel) and $B_{z}$ (bottom panel) as a function of space and time, for $\beta \mu=5$ and $T_{\perp} / T_{\|}=2$. The magnetic field has been normalized by $\omega_{p e} m_{e} / e$. We see a nonlinear saturation of the magnetic field components at an amplitude of $\sim 0.01$. After Ref. [183].
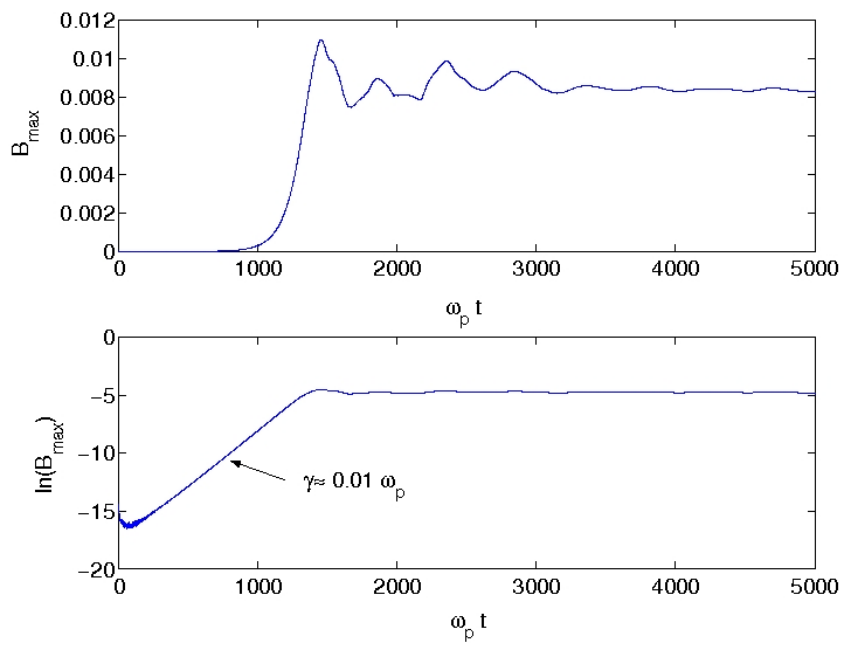

FIG. 16: The maximum of the magnetic field amplitude, $B=\left(B_{y}^{2}+B_{z}^{2}\right)^{1 / 2}$, over the simulation box (top panel), and the logarithm of the magnetic field maximum (bottom panel) as a function of time, for $T_{\perp} / T_{\|}=2$ and $\beta \mu=5$. The magnetic field has been normalized by $\omega_{p e} m_{e} / e$. From the logarithmic slope of the magnetic field in the linear regime one finds $\gamma \approx \Delta \ln \left(B_{\max }\right) / \Delta t \approx 0.01 \omega_{p}$. After Ref. [183.

For a Maxwellian plasma, it has been found [196] that the Weibel instability saturates nonlinearly once the magnetic bounce frequency $\omega_{c}=e B / m_{e} c$ has increased to a value comparable to the linear growth rate. In order to assess the nonlinear behavior of the Weibel instability for a degenerate plasma, we have carried out a kinetic simulation of the Wigner-Maxwell system. We have assumed that the quantum diffraction effect is small, so that the simulation of the Wigner equation can be approximated by simulations of the Vlasov equation by means of an electromagnetic Vlasov code [197. As an initial condition for the simulation, we used the distribution function 896. In order to give a seed for any instability, the plasma density was perturbed with low-frequency fluctuations (random numbers). The results are displayed in Figs. 15 and 16 for the parameters $\beta \mu=5$ and $T_{\perp} / T_{\|}=2$, corresponding to the solid lines in Figs. 13 and 14. Figure 15 shows the magnetic field components as a function of space and time. We see that the magnetic field initially grows, and saturates to steady state magnetic field fluctuations with an amplitude of $e B / m_{e} c \omega_{p e} \approx 0.008$. The maximum amplitude of the magnetic field over the simulation box as a function of time is shown in Fig. 16. where we see that the magnetic field saturates at $e B / m_{e} \omega_{p e} \approx 0.0082$, while the linear growth rate of the most unstable mode is $\gamma_{\max } / \omega_{p e} \approx 0.009$. Similar to the classical Maxwellian plasma case [196], we can thus 
estimate the generated magnetic field as

$$
B=\frac{m_{e} c \gamma_{\max }}{e}
$$

for a degenerate Fermi plasma. For our parameters parameters relevant for intense laser-solid interaction experiments, we will thus have magnetic fields of the order $10^{5}$ Tesla (one gigagauss).

\section{B. Dense plasma magnetization by the electromagnetic wave}

The second example of the dense plasma magnetization can occur in the presence of streaming electrons and large amplitude electromagnetic waves. Here, the nonstationary ponderomotive force of the electromagnetic wave would create slowly varying electric fields and currents, which generate d.c. magnetic fields in dense plasmas.

Let us consider the propagation of the electromagnetic wave with the electric field $\mathbf{E}(\mathbf{r}, t)=(1 / 2) \mathbf{E}_{0}(x, t) \exp (-i \omega t+$ $i k x)+$ c.c., in an unmagnetized non-relativistic dense plasma with streaming electrons (with the drift velocity $u \hat{\mathbf{z}}$, where $u$ is the magnitude of the electron drift speed and $\hat{\mathbf{z}}$ is the unit vector along the $z$ axis in a Cartesian coordinate system; typically $u$ is much smaller than the electron Fermi speed) and immobile ions. Here, $\mathbf{E}_{0}(x, t)$ is the envelope of the electromagnetic field at the position $\mathbf{r}$ and time $t$, and c.c. stands for complex conjugate. The frequency $\omega$ and the wave vector $\mathbf{k}=k \hat{\mathbf{x}}$, where $\hat{\mathbf{x}}$ is the unit vector along the $x$ axis, are related by 198 .

$$
\frac{k^{2} c^{2}}{\omega^{2}}=N=1-\frac{\omega_{p e}^{2}}{\omega^{2}}-\frac{k^{2} u^{2} \omega_{p e}^{2}}{\omega^{2}\left(\omega^{2}-k^{2} V_{F e}^{2}-\Omega_{q}^{2}\right)}
$$

where $N$ is the index of refraction and $\Omega_{q}=\hbar k^{2} / 2 m_{e}$,

The electromagnetic wave exerts a ponderomotive force $\mathbf{F}_{p}=\mathbf{F}_{p s}+\mathbf{F}_{p t}$ on the plasma electrons, where the stationary and non-stationary ponderomotive forces [173] are, respectively,

$$
\mathbf{F}_{p s}=\frac{(N-1)}{16 \pi} \nabla\left|\mathbf{E}_{0}\right|^{2}
$$

and

$$
\mathbf{F}_{p t}=\frac{1}{16 \pi} \frac{\mathbf{k}}{\omega^{2}} \frac{\partial\left[\omega^{2}(N-1)\right]}{\partial \omega} \frac{\partial\left|\mathbf{E}_{0}\right|^{2}}{\partial t}
$$

The ponderomotive force pushes the electrons locally, and creates the slowly varying electric field

$$
\mathbf{E}_{s}=-\nabla \phi_{s}-\frac{1}{c} \frac{\partial \mathbf{A}_{s}}{\partial t}=\frac{1}{n_{0} e} \mathbf{F}_{p}
$$

where the scalar and vector potentials are, respectively,

$$
\phi_{s}=-\frac{(N-1)}{16 \pi n_{0} e}\left|\mathbf{E}_{0}\right|^{2}
$$

and

$$
\mathbf{A}_{s}=-\frac{c}{16 \pi n_{0} e} \frac{\mathbf{k}}{\omega^{2}} \frac{\partial\left[\omega^{2}(N-1)\right]}{\partial \omega}\left|\mathbf{E}_{0}\right|^{2}
$$

The induced slowly varying magnetic field $\mathbf{B}_{s}$ is then $\mathbf{B}_{s}=\nabla \times \mathbf{A}_{s}$. Noting that

$$
\frac{\partial\left[\omega^{2}(N-1)\right]}{\partial \omega}=\frac{2 \omega k^{2} u^{2} \omega_{p e}^{2}}{\left(\omega^{2}-k^{2} V_{F e}^{2}-\Omega_{q}^{2}\right)^{2}}
$$


we can express the magnitude of the magnetic field as

$$
\left|\mathbf{B}_{s}\right|=\frac{e c k^{3} u^{2}\left|\mathbf{E}_{0}\right|^{2}}{2 m_{e} L \omega\left(\omega^{2}-\Omega^{2}\right)^{2}}
$$

where $L$ is scale length of the envelope $\left|\mathbf{E}_{0}\right|^{2}$ and $\Omega=\left(k^{2} V_{F e}^{2}+\Omega_{q}^{2}\right)^{1 / 2} \equiv\left(\hbar k^{2} / 2 m_{e}\right)\left[1+4\left(3 \pi^{2} n_{0}\right)^{2 / 3} / k^{2}\right]^{1 / 2}$. We note from 102 that the magnetic field strength is proportional to $u^{2}$, and attains a large value when $\omega \sim \Omega$. The electron gyrofrequency $\Omega_{c}$ is

$$
\Omega_{c}=\frac{e\left|\mathbf{B}_{s}\right|}{m_{e} c}=\frac{k^{3} V_{0}^{2} u^{2} \omega}{2 L\left(\omega^{2}-\Omega^{2}\right)^{2}},
$$

where $V_{0}=e\left|\mathbf{E}_{0}\right| / m_{e} \omega$ is the electron quiver velocity in the electromagnetic field.

\section{DYNAMICS OF ELECTROMAGNETIC WAVES IN DENSE PLASMAS}

We here consider various electromagnetic (EM) wave modes and their nonlinear interaction in dense plasmas. As examples, we will discuss spin waves, which occur in a magnetized plasma due to the spin-1/2 effect of electrons and positrons. We also consider nonlinear interactions between finite amplitude electromagnetic and electrostatic waves in dense plasmas. We focus on the underlying physics of stimulated scattering instabilities and the wave localization due to the parametric interactions involving the radiation pressure [178.

\section{A. Electromagnetic spin waves in magnetized plasmas}

In the presence of an external magnetic field, the quantum description of the linear kinetics of a dense collisionless plasma is complicated due to quantization of the gyromagnetic motion and the inclusion of the electron and positron spin-1/2. In the past, many authors [199, 200, 201, 202, 203. investigated the high-frequency conductivity, longitudinal and transverse dielectric responses in dense magnetized plasmas. The propagation characteristics of high-frequency electromagnetic waves in quantum magnetoplasmas is different from that in a classical magnetoactive plasma [204.

Recently, Oraevsky et al. 70] found a new electromagnetic spin wave whose electric field is parallel to $\hat{\mathbf{z}} B_{0}$, and which propagates across $\hat{\mathbf{z}}$, where $\hat{\mathbf{z}}$ is the unit vector along the $z$ axis in a Cartesian coordinate system and $B_{0}$ is the strength of the ambient magnetic field. The spin wave accompany the magnetization current due to spinning electron motion and the wave frequency is obtained from [70]

$$
\frac{k_{\perp}^{2} c^{2}}{\omega^{2}}=1-\frac{\omega_{p e}^{2}}{\omega\left(\omega+i \nu_{e}\right)}+2 \pi \mu_{B}^{2} \frac{k_{\perp}^{2} c^{2} n_{0}}{\omega^{2} \epsilon_{F}} \frac{\omega_{c e}}{\omega-\omega_{c e}},
$$

where $k_{\perp}$ is the perpendicular component of the wave vector $\mathbf{k}=\hat{\mathbf{x}} k_{\perp}, \hat{\mathbf{x}}$ is the unit vector transverse to $\hat{\mathbf{z}}, \nu_{e}$ is the electron collision frequency, nd $\epsilon_{F} \sim m_{e} c^{2}$. The third term in the right-hand side of (102) represents the electron spin-magnetic resonance at the electron gyrofrequency $\omega_{c e}=e B_{0} / m_{e} c$.

Assuming that $k_{\perp}^{2} c^{2}+\omega_{p e}^{2} \neq \omega_{c e}^{2}$, we obtain from (102)

$$
\omega \simeq \omega_{c e}\left[1+\frac{2 \pi \mu_{B}^{2} n_{0}}{\epsilon_{F}} \frac{k_{\perp}^{2} c^{2}}{k_{\perp}^{2} c^{2}+\omega_{p e}^{2}\left(1-i \nu_{e} / \omega\right)-\omega_{c e}^{2}}\right] .
$$

For $\nu_{e} \ll \omega \sim \omega_{c e}$, the damping rate of the spin mode is

$$
\operatorname{Im} \omega \simeq \frac{\nu_{e} \omega_{p e}^{2} k_{\perp}^{2} c^{2} \mu_{B}^{2} B_{0}^{2}}{\epsilon_{F} m_{e} c^{2} \omega_{c e}^{2}\left(k_{\perp}^{2} c^{2}+\omega_{p e}^{2}-\omega_{c e}^{2}\right)^{2}}
$$

The ponderomotive force of the spin wave can create compressional magnetic field perturbation due to an inverse Cotton-Mouton/Faraday effect [205]. 


\section{B. Nonlinearly coupled EM waves}

Finite amplitude electromagnetic waves in quantum magnetoplasmas interact nonlinearly among themselves. In this subsection, we shall use the generalized Q-MHD equations to obtain compact nonlinear equations for the electron magnetohydrodynamic (EMHD) and the Hall-MHD plasmas, and show how does the density, the fluid velocity, and magnetic field perturbations are coupled in a non-trivial manner.

The governing nonlinear equations for the electromagnetic waves in dense magnetoplasmas are the quantum magnetohydrodynamic equations composed of the continuity equation

$$
\frac{\partial n_{e, i}}{\partial t}+\nabla \cdot\left(n_{e, i} \mathbf{u}_{e, i}\right)=0
$$

the electron and ion momentum equations, respectively,

$$
\begin{gathered}
n_{e} m_{e}\left(\frac{\partial}{\partial t}+\mathbf{u}_{e} \cdot \nabla\right) \mathbf{u}_{e}=-n_{e} e\left(\mathbf{E}+\frac{1}{c} \mathbf{u}_{e} \times \mathbf{B}\right)-\nabla P_{e}+\mathbf{F}_{Q e} \\
n_{i} m_{i}\left(\frac{\partial}{\partial t}+\mathbf{u}_{i} \cdot \nabla\right) \mathbf{u}_{i}=Z_{i} e n_{i}\left(\mathbf{E}+\frac{1}{c} \mathbf{u}_{i} \times \mathbf{B}\right)
\end{gathered}
$$

the Faraday law

$$
c \nabla \times \mathbf{E}=-\frac{\partial \mathbf{B}}{\partial t}
$$

the Maxwell equation including the magnetization spin current

$$
\nabla \times \mathbf{B}=\frac{4 \pi}{c}\left(\mathbf{J}_{p}+\mathbf{J}_{m}\right)+\frac{1}{c} \frac{\partial \mathbf{E}}{\partial t},
$$

where the pressure for non-relativistic degenerate electrons reads 206]

$$
P_{e}=\frac{4 e B\left(2 m_{e}\right)^{1 / 2} E_{F}^{3 / 2}}{3(2 \pi)^{2} \hbar^{2} c}\left[1+2 \sum_{n_{L}=1}^{n_{\max }}\left(1-\frac{n_{L} \hbar \omega_{c e}}{E_{F}}\right)^{3 / 2}\right]
$$

where $n_{L}=0,1,2, \ldots, n_{\max }$, and the value of $n_{\max }$ is fixed by the largest integer $n_{L}$ that satisfies $E_{F}-n_{L} \hbar \omega_{c e} \geq 0$.

The sum of the quantum Bohm and intrinsic angular momentum spin forces is

$$
\mathbf{F}_{Q e}=\nabla\left(\frac{\nabla^{2} \sqrt{n_{e}}}{\sqrt{n_{e}}}\right)-\frac{n_{e} \mu_{B}^{2}}{k_{B} T_{F e}} \nabla B
$$

In Eqs. (107)-113), $n_{j}$ is the number density of the particle species $j$ ( $j$ equals $e$ for the electrons, and $i$ for the ions), $\mathbf{u}_{j}$ is the particle fluid velocity, and $B=|\mathbf{B}|$. We have denoted the plasma current density $\mathbf{J}_{p}=-n_{e} e \mathbf{u}_{e}+Z_{i} n_{i} e \mathbf{u}_{i}$ and the electron magnetization spin current density $\mathbf{J}_{m}=\nabla \times \mathbf{M}$, where the magnetization for dynamics on a time scale much slower than the spin precession frequency for an electron Fermi gas reads $[208] \mathbf{M}=\left(n_{e} \mu_{B}^{2} / k_{B} T_{F e}\right) \hat{\mathbf{B}}$.

\section{Nonlinear EMHD}

First, we present the generalized nonlinear EMHD equations for a dense magnetoplasma. Here the ions form the neutralizing background. The wave phenomena in the EMHD plasma will occur on a time scale much shorter than the ion plasma and ion gyroperiods. In equilibrium, we have

$$
n_{e 0}=Z_{i} n_{i 0} \equiv n_{0}
$$


The relevant nonlinear EMHD equations are

$$
\frac{\partial n_{e}}{\partial t}+\nabla \cdot\left(n_{e} \mathbf{u}_{e}\right)=0
$$

the electron momentum equation 108, Faraday's law (110), and the electron fluid velocity given by

$$
\mathbf{u}_{e}=\frac{\mathbf{J}_{m}}{e n_{e}}-\frac{c(\nabla \times \mathbf{B})}{4 \pi e n_{e}}+\frac{1}{e n_{e}} \frac{\partial \mathbf{E}}{\partial t} .
$$

We observe that the quantum tunneling and spin forces play an important role if there are slight electron density and magnetic field inhomogeneities in dense plasmas. The nonlinear EMHD equations are useful for studying collective electron dynamics in metallic and semiconductor nanostructures [112].

\section{Nonlinear Hall-MHD}

Second, we derive the modified nonlinear Hall-MHD equations in a dense electron-ion plasma. The Hall-MHD equations shall deal with the wave phenomena on a time scale larger than the electron gyroperiod. The relevant nonlinear Hall-MHD equations are the electron and ion continuity equations, the inertialess electron momentum equation

$$
\mathbf{E}+\frac{1}{c} \mathbf{u}_{e} \times \mathbf{B}-\frac{\nabla P_{e}}{n_{e} e}-\frac{\mathbf{F}_{Q e}}{n_{e} e}=0,
$$

Faraday's law 110, the ion momentum equation

$$
n_{i} m_{i}\left(\frac{\partial}{\partial t}+\mathbf{u}_{i} \cdot \nabla\right) \mathbf{u}_{i}=Z_{i} e n_{i}\left(\mathbf{E}+\frac{1}{c} \mathbf{u}_{i} \times \mathbf{B}\right),
$$

and the electron fluid velocity given by

$$
\mathbf{u}_{e}=\mathbf{u}_{i}+\frac{\mathbf{J}_{m}}{e n_{e}}-\frac{c(\nabla \times \mathbf{B})}{4 \pi e n_{e}}
$$

where we have neglected the displacement current, since the Hall-MHD plasma deals with electromagnetic waves whose phase velocity is much smaller than the speed of light in vacuum.

We now eliminate the electric field from (118) by using [117, obtaining

$$
n_{i} m_{i}\left(\frac{\partial}{\partial t}+\mathbf{u}_{i} \cdot \nabla\right) \mathbf{u}_{i}=Z_{i} e n_{i}\left[\frac{1}{c}\left(\mathbf{u}_{i}-\mathbf{u}_{e}\right) \times \mathbf{B}-\frac{\nabla P_{e}}{n_{e} e}+\frac{\mathbf{F}_{Q e}}{n_{e} e}\right] .
$$

Furthermore, eliminating $\mathbf{u}_{e}$ from 120 by using 1119 , we have

$$
n_{i} m_{i}\left(\frac{\partial}{\partial t}+\mathbf{u}_{i} \cdot \nabla\right) \mathbf{u}_{i}=Z_{i} e n_{i}\left\{\frac{1}{c}\left[-\frac{\mathbf{J}_{m}}{e n_{e}}+\frac{c(\nabla \times \mathbf{B})}{4 \pi}\right] \times \mathbf{B}-\frac{\nabla P_{e}}{n_{e} e}+\frac{\mathbf{F}_{Q e}}{n_{e} e}\right\},
$$

with the quasi-neutrality condition $n_{e}=Z_{i} n_{i}$.

Finally, by using (117) we can eliminate $\mathbf{E}$ from 108 , obtaining

$$
\frac{\partial \mathbf{B}}{\partial t}=\nabla \times\left[\left(\mathbf{u}_{i} \times \mathbf{B}\right)+\frac{\mathbf{J}_{m} \times \mathbf{B}}{e Z_{i} n_{i}}-\frac{c}{4 \pi}(\nabla \times \mathbf{B}) \times \mathbf{B}\right] .
$$

The ion continuity equation, Eqs. (121) and (122), together with 118 and $Z_{i} n_{i 1}=n_{e 1}$, where $n_{e 1, i 1} \ll n_{e 0, i 0}$ are the desired generalized nonlinear equations for the low-frequency (in comparison with the electron gyrofrequency), low-phase velocity (in comparison with the speed of light in vacuum) in a Hall-MHD dense plasma. They can be used to investigate the multi-dimensional linear and nonlinear waves (e.g. magnetosonic solitons [209]), as well as nanostructures and turbulences [210] in dense quantum magnetoplasmas. 


\section{Stimulated scattering instabilities}

Nonlinear interactions between the high-frequency EM waves and low-frequency electrostatic waves give rise to stimulated scattering instabilities in classical plasmas [130, 211, 212, 213. There also exists possibility of exciting plasma waves by the high-frequency EM waves in quantum plasmas due to the parametric instabilities [214]. The governing equations for the high-frequency electromagnetic waves 64 and the radiation pressure driven modified Langmuir and ion-acoustic oscillations in an unmagnetized quantum plasma are, respectively,

$$
\begin{gathered}
\left(\frac{\partial^{2}}{\partial t^{2}}-c^{2} \nabla^{2}+\omega_{p e}^{2}\right) \mathbf{A}+\omega_{p e}^{2} \frac{n_{1}}{n_{0}} \mathbf{A} \approx 0 \\
\left(\frac{\partial^{2}}{\partial t^{2}}+\omega_{p e}^{2}-\frac{3}{5} V_{F e}^{2} \nabla^{2}+\frac{\hbar^{2}}{4 m_{e}^{2}} \nabla^{4}\right) \frac{n_{1}}{n_{0}}=\frac{q_{e}^{2}}{2 m_{e}^{2} c^{2}} \nabla^{2}|\mathbf{A}|^{2},
\end{gathered}
$$

and

$$
\left(\frac{\partial^{2}}{\partial t^{2}}-\frac{m_{e}}{m_{i}} V_{F e}^{2} \nabla^{2}+\frac{\hbar^{2}}{4 m_{e} m_{i}} \nabla^{4}\right) \frac{n_{1}}{n_{0}}=\frac{q_{e}^{2}}{2 m_{e} m_{i} c^{2}} \nabla^{2}|\mathbf{A}|^{2}
$$

where $\mathbf{A}$ is the vector potential of the high-frequency electromagnetic wave, and $n_{1}$ is the electron density perturbation of the low-frequency oscillations (viz. the modified EPOs and ion waves).

Combining Eqs. 123)-125 we thus simply obtain the nonlinear dispersion relations

$$
\omega^{2}-\Omega_{R}^{2}=-\frac{q_{e}^{2} \omega_{p e}^{2} k^{2}\left|\mathbf{A}_{0}\right|^{2}}{2 m_{e}^{2} c^{2}} \sum_{ \pm} \frac{1}{D_{ \pm}},
$$

and

$$
\omega^{2}-\Omega_{B}^{2}=-\frac{q_{e}^{2} \omega_{p e}^{2} k^{2}\left|\mathbf{A}_{0}\right|^{2}}{2 m_{e} m_{i} c^{2}} \sum_{ \pm} \frac{1}{D_{ \pm}},
$$

where $\Omega_{R}=\left(\omega_{p e}^{2}+3 k^{2} V_{F e}^{2} / 5+\hbar^{2} k^{4} / 4 m_{e}^{2}\right)^{1 / 2}$ and $\Omega_{B}=\left(k^{2} C_{F s}^{2}+\hbar^{2} k^{4} / m_{e} m_{i}\right)^{1 / 2}$.

Equations (126) and (127) admit stimulated Raman, stimulated Brillouin and modulational instabilities of the electromagnetic pump (with the amplitude $\mathbf{A}_{0}$ and the frequency $\omega_{0}=\left(k_{0}^{2} c^{2}+\omega_{p e}^{2}\right)^{1 / 2}$ ) in dense quantum plasmas. We have denoted $D_{ \pm}= \pm 2 \omega_{0}\left(\omega-\mathbf{k} \cdot \mathbf{V}_{g} \mp \delta\right)$, where $\mathbf{V}_{g}=c^{2} \mathbf{k}_{0} / \omega_{0}$ is the group velocity of the EM pump wave frequency, and $\delta=k^{2} c^{2} / 2 \omega_{0}$ is the small frequency shift arising from the nonlinear interaction of the pump with the electrostatic perturbations $(\omega, \mathbf{k})$ in our quantum plasma. For stimulated Raman and Brillouin scatterings, the EM pump wave is scattered off the resonant electron plasma wave and resonant ion wave, respectively, while for the modulational instability the electron and ion plasma oscillations are off resonant, so are the EM sidebands.

For three-wave decay interactions, we suppose that $D_{-}=0$ and $D_{+} \neq 0$. Thus, we ignore $D_{+}$from $(126)$ and (127). Letting $\omega=\Omega_{R}+i \gamma_{R}$ and $\omega=\Omega_{B}+i \gamma_{B}$ in (126) and (127), respectively, and assuming $\omega-\mathbf{k} \cdot \mathbf{V}_{g}+\delta \equiv i \gamma_{R, B}$, we obtain the the growth rates for stimulated Raman and Brillouin scattering instabilities (denoted by the subscripts $R$ and $B$, respectively,

$$
\gamma_{R}=\frac{\omega_{p e} e k\left|\mathbf{A}_{0}\right|^{2}}{2 \sqrt{2 \omega_{0} \Omega_{R}} m_{e} c}
$$

and

$$
\gamma_{B}=\frac{\omega_{p e} e k\left|\mathbf{A}_{0}\right|^{2}}{2 \sqrt{2 \omega_{0} \Omega_{B} m_{e} m_{i}} c}
$$

where $\left|\mathbf{k} \cdot \mathbf{V}_{g}-\delta\right| \sim \Omega_{R}, \Omega_{B}$. We note that the growth rates for stimulated Raman and Brillouin scattering instabilities are inversely proportional to the square roots of $\Omega_{R}$ and $\Omega_{B}$, which depend on the quantum parameters. Stimulated Raman and Brillouin scattering instabilities of the EM wave off the ES waves should provide invaluable information regarding the density fluctuations and equation of states that might exist in dense quantum plasmas.

The quantum corrected 3D Zakharov equations [127] have been derived by Haas [215] and Haas and Shukla [216], who demonstrated that the dispersive effects associated with quantum corrections can prevent the collapse of localized Langmuir envelope electric fields in both two and three spatial dimensions. 


\section{Self-trapped EM waves in a quantum hole}

Nonlinear interactions between large amplitude electromagnetic waves and electrostatic plasma waves can produce nonlinear nanostructures composed of a density cavity that traps the electromagnetic wave envelope. Here we demonstrate trapping of intense electromagnetic waves into a finite amplitude density hole 65] arising at the scale-size of the order of the electron skin depth $\left.c / \omega_{p e}\right)$.

A powerful circularly polarized electromagnetic (CPEM) plane wave interacting nonlinearly with the EPOs would generate an envelope of the CPEM vector potential $\mathbf{A}_{\perp}=A_{\perp}(\hat{\mathbf{x}}+i \hat{\mathbf{y}}) \exp \left(-i \omega_{0} t+i k_{0} z\right)$, which shall obey the nonlinear Schrödinger equation 178

$$
2 i \Omega_{0}\left(\frac{\partial}{\partial t}+V_{g} \frac{\partial}{\partial z}\right) A_{\perp}+\frac{\partial^{2} A_{\perp}}{\partial z^{2}}-\left(\frac{|\psi|^{2}}{\gamma}-1\right) A_{\perp}=0
$$

where the electron wave function $\psi$ and the scalar potential are governed by, respectively,

$$
i H_{e} \frac{\partial \psi}{\partial t}+\frac{H_{e}^{2}}{2} \frac{\partial^{2} \psi}{\partial z^{2}}+(\phi-\gamma+1) \psi=0
$$

and

$$
\frac{\partial^{2} \phi}{\partial z^{2}}=|\psi|^{2}-1
$$

where the electron number density is defined as is given by the $|\psi|^{2}$ term. Here $\Omega_{0}$ represents the CPEM wave frequency, $V_{g}$ is the $x$ component of the group velocity of the CPEM wave, $H_{e}$ is a quantum coupling parameter, and $\gamma=\left(1+\left|A_{\perp}\right|^{2}\right)^{1 / 2}$ is the relativistic gamma factor due to the electron quiver velocity in the CPEM wave fields. The details of normalization of variables is given in Ref. 65. The nonlinear coupling between intense CPEM waves and EPOs comes about due to the nonlinear current density, which is represented by the term $|\psi|^{2} A_{\perp} / \gamma$ in Eq. 130 . In Eq. (131], $1-\gamma$ is the relativistic ponderomotive potential [178, which arises due to the cross-coupling between the CPEM wave-induced electron quiver velocity and the CPEM wave magnetic field.

The effect of quantum dispersion on localized electromagnetic pulses can be studied by considering a steady state structure moving with a constant speed $V_{g}$. Inserting the ansatz $A_{\perp}=W(\xi) \exp (-i \Omega t), \psi=P(\xi) \exp (i k x-i \omega t)$ and $\phi=\phi(\xi)$ into Eqs. $130-132$, where $\xi=z-V_{g} t, k=V_{g} / H_{e}$ and $\omega=V_{g}^{2} / 2 H_{e}$, and where $W(\xi)$ and $P(\xi)$ are real, one obtains from $130-132$ the coupled system of equations

$$
\begin{gathered}
\frac{\partial^{2} W}{\partial \xi^{2}}+\left(\lambda-\frac{P^{2}}{\gamma}+1\right) W=0 \\
\frac{H_{e}^{2}}{2} \frac{\partial^{2} P}{\partial \xi^{2}}+(\phi-\gamma+1) P=0
\end{gathered}
$$

where $\gamma=\left(1+W^{2}\right)^{1 / 2}$, and

$$
\frac{\partial^{2} \phi}{\partial \xi^{2}}=P^{2}-1
$$

with the boundary conditions $W=\Phi=0$ and $P^{2}=1$ at $|\xi|=\infty$. In Eq. $(133), \lambda=2 \Omega_{0} \Omega$ represents a nonlinear frequency shift of the CPEM wave. In the limit $H_{e} \rightarrow 0$, one has from $1134 \phi=\gamma-1$, where $P \neq 0$, and one recovers the classical (non-quantum) case of the relativistic solitary waves in a cold plasma [217]. The system of equations (133) - 135 admits a Hamiltonian

$$
Q_{H}=\frac{1}{2}\left(\frac{\partial W}{\partial \xi}\right)^{2}+\frac{H_{e}^{2}}{2}\left(\frac{\partial P}{\partial \xi}\right)^{2}-\frac{1}{2}\left(\frac{\partial \phi}{\partial \xi}\right)^{2}+\frac{1}{2}(\lambda+1) W^{2}+P^{2}-\gamma P^{2}+\phi P^{2}-\phi=0
$$

where the boundary conditions $\partial / \partial \xi=0, W=\phi=0$ and $|P|=1$ at $|\xi|=\infty$ have been used.

Numerical solutions of the quasi-stationary system $\sqrt{133}-(135)$ are presented Figs. 17 and 18 , while time-dependent solutions of Eqs. 130-132 are shown in Figs. 19 and 20. Here parameters were used that are representative of the next generation laser-based plasma compression (LBPC) schemes [13, 14, 81]. The formula [178] $e A_{\perp} / m c^{2}=$ $6 \times 10^{-10} \lambda_{s} \sqrt{I}$ will determine the normalized vector potential, provided that the CPEM wavelength $\lambda_{s}$ (in microns) 
and intensity $I$ (in $\mathrm{W} / \mathrm{cm}^{2}$ ) are known. It is expected that in LBPC schemes, the electron number density $n_{0}$ may reach $10^{27} \mathrm{~cm}^{-3}$ and beyond, and the peak values of $e A_{\perp} / m c^{2}$ may be in the range 1-2 (e.g. for focused EM pulses with $\lambda_{s} \sim 0.15 \mathrm{~nm}$ and $\left.I \sim 5 \times 10^{27} \mathrm{~W} / \mathrm{cm}^{2}\right)$. For $\omega_{p e}=1.76 \times 10^{18} \mathrm{~s}^{-1}$, one has $\hbar \omega_{p e}=1.76 \times 10^{-9}$ erg and $H_{e}=0.002$, since $m c^{2}=8.1 \times 10^{-7} \mathrm{erg}$. The electron skin depth $\lambda_{e} \sim 1.7 \AA$. On the other hand, a higher value of $H_{e}=0.007$ is achieved for $\omega_{p e}=5.64 \times 10^{18} \mathrm{~s}^{-1}$. Thus, our numerical solutions below, based on these two values of $H_{e}$, have focused on scenarios that are relevant for the next generation intense laser-solid density plasma interaction experiments 81 .

Figures 17 and 18 show numerical solutions of Eqs. (133)- 135 for several values of $H_{e}$. The nonlinear boundary value problem was solved with the boundary conditions $W=\phi=0$ and $P=1$ at the boundaries at $\xi= \pm 10$. One can see that the solitary envelope pulse is composed of a single maximum of the localized vector potential $W$ and a local depletion of the electron density $P^{2}$, and a localized positive potential $\phi$ at the center of the solitary pulse. The latter has a continuous spectrum in $\lambda$, where larger values of negative $\lambda$ are associated with larger amplitude solitary EM pulses. At the center of the solitary EM pulse, the electron density is partially depleted, as in panels a) of Fig. 17, and for larger amplitudes of the EM waves one has a stronger depletion of the electron density, as shown in panels b) and c) of Fig. 17. For cases where the electron density goes to almost zero in the classical case [217], one important quantum effect is that the electrons can tunnel into the depleted region. This is seen in Fig. 18, where the electron density remains nonzero for the larger value of $H_{e}$ in panels a), while the density shrinks to zero for the smaller value of $H_{e}$ in panel b).
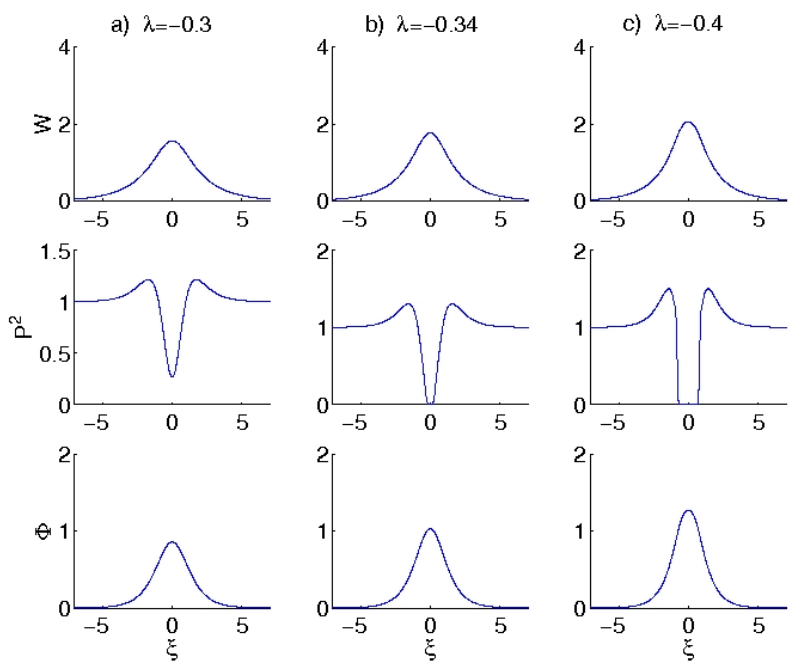

FIG. 17: The profiles of the CPEM vector potential $W$ (top row), the electron number density $P^{2}$ (middle row) and the scalar potential $\Phi$ (bottom row) for $\lambda=-0.3$ (left column), $\lambda=-0.34$ (middle column) and $\lambda=-0.4$ (right column), with $H_{e}=0.002$. After Ref. 65.

Figures 19 and 20 show numerical simulation results of Eqs. $130-132$, in order to investigate the quantum diffraction effects on the dynamics of localized CPEM wavepackets. Here the long-wavelength limit $\omega_{0} \approx 1$ and $V_{g} \approx 0$ was considered. In the initial conditions, an EM pump with a constant amplitude $A_{\perp}=A_{0}=1$ and a uniform plasma density $\psi=1$ was used, together with a small amplitude noise (random numbers) of order $10^{-2}$ added to $A_{\perp}$ to give a seeding any instability. The numerical results are displayed in Figs. 19 and 20 for $H_{e}=0.002$ and $H_{e}=0.007$, respectively. In both cases, one can see an initial linear growth phase and a wave collapse at $t \approx 70$, in which almost all the CPEM wave energy is contracted into a few well separated localized CPEM wave pipes. These are characterized by a large bell-shaped amplitude of the CPEM wave, an almost complete depletion of the electron number density at the center of the CPEM wavepacket, and a large-amplitude positive electrostatic potential. Comparing Fig. 19 with Fig. 20, one can see that there is a more complex dynamics in the interaction between the CPEM wavepackets for the larger $H_{e}=0.007$, shown in Fig. 20, in comparison with $H_{e}=0.002$, shown in Fig. 19, where the wavepackets are almost stationary when they are fully developed.

\section{SUMMARY AND PERSPECTIVES}

In this paper, we have presented our up-to-date theoretical knowledge of nonlinear physics of non-relativistic quantum plasmas. We started with nonlinear quantum models that describe the physics of localized excitations in 
a) $\mathrm{He}=0.007$
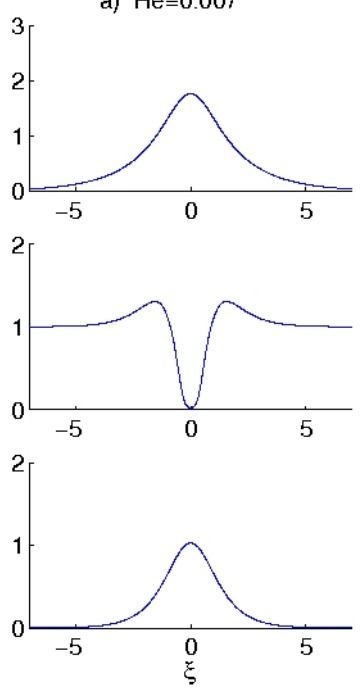

b) $\mathrm{He}=0.002$
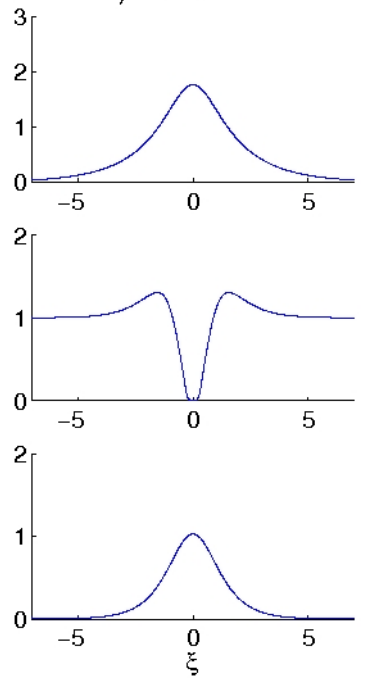

FIG. 18: The profiles of the CPEM vector potential $W$ (top row), the electron number density $P^{2}$ (middle row) and the scalar potential $\Phi$ (bottom row) for $H_{e}=0.007$ (left column) and $H_{e}=0.002$ (right column), with $\lambda=-0.34$. After Ref. [65].

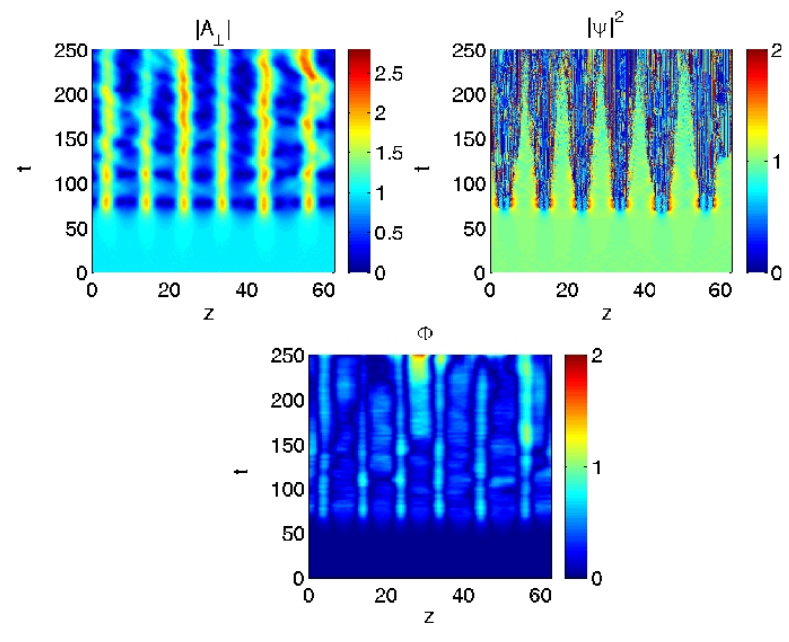

FIG. 19: The dynamics of the CPEM vector potential $A_{\perp}$ and the electron number density $|\psi|^{2}$ (upper panels) and of the electrostatic potential $\Phi$ (lower panel) for $H_{e}=0.002$. After Ref. 65].

different areas of physics. We then moved to discussing the well known electron and ion plasma wave spectra in dense quantum plasmas, and presented nonlinear models for treating nonlinear interactions among finite amplitude plasma waves at nanoscales. As examples, we demonstrated the existence of localized nonlinear EPOs and localized ion waves in dense quantum plasmas. For electrostatic EPOs, the electron dynamics is governed by a pair composed of nonlinear Schrödinger and Poisson (NLSP) equations. We stress that the nonlinear Schrödinger equation governing the spatio-temporal evolution of the electron wave function in the presence of self-consistent electrostatic potential in dense plasmas has been obtained from the quantum electron momentum equation by introducing the eikonal representation as a mathematical tool for understanding the complex electron plasma wave interactions at nanoscales. Such a description also appears in the context of the nonlinear electron dynamics in thin metal films [112. Our NLSP equations admit a set of conserved quantities, e.g. the total number of electrons, the electron momentum, the electron angular momentum, and the electron energy. We have found that the NLSP equations admit quasistationary, localized structures in the form of one-dimensional quantized dark solitons and two-dimensional quantized vortices. These nanostructures are associated with a local depletion of the electron density associated with positive electrostatic potential, and are parameterised by the quantum coupling parameter only. In the two-dimensional geometry, there exist a class of vortices of different excited states (charge states) associated with a complete depletion 


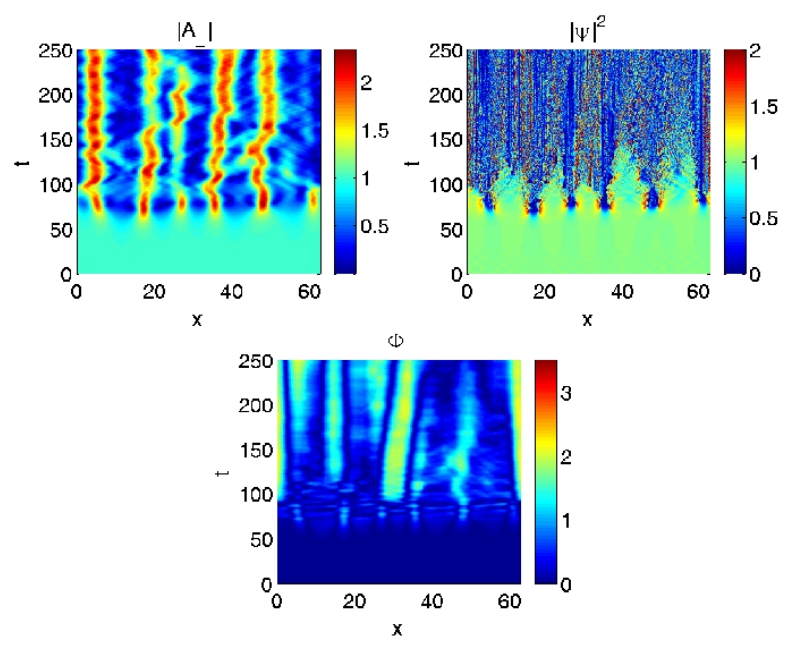

FIG. 20: The dynamics of the CPEM vector potential $A_{\perp}$ and the electron number density $|\psi|^{2}$ (upper panels) and the electrostatic potential $\phi$ (lower panel) for $H_{e}=0.007$. After Ref. 65].

of the electron density and an associated positive potential. Numerical simulations of the time-dependent NLSP equations demonstrate the stability of stable dark solitons in one space dimension with an amplitude consistent with the one found from the time-independent solutions. In two-space-dimensions, the dark solitons of the first excited state were found to be stable and the preferred nonlinear state was in the form of vortex pairs of different polarities. One-dimensional dark solitons and singly charge two-dimensional vortices are thus long-lived nonlinear structures in quantum plasmas.

Similar to the Bernstein-Green-Kruskal modes [164, we accounted for the trapping of electrons in the electrostatic wave potential and studied numerically the deformation of the equilibrium Fermi-Dirac distribution function and the subsequent emergence of localized phase space kinetic structures. Such an investigation was carried out by using the time-dependent Wigner and Poisson equations. Furthermore, we presented two mechanisms for the generation of magnetic fields in quantum plasmas. They are associated with the quantum Weibel instability in the presence of equilibrium anisotropic Fermi-Dirac electron distribution function, as well as with the the non-stationary ponderomotive force of a large amplitude electromagnetic waves in quantum plasmas with streaming electrons. Spontaneously generated magnetic fields can affect the linear and nonlinear propagation of both the electrostatic and electromagnetic waves in quantum magnetoplasmas 39 . The quantum corrections produce dispersion at short scales for the electrostatic upper-hybrid, lower-hybrid, and ion-cyclotron waves, while the quantum Bohm force and the electron-1/2 spin effects introduce new features to the elliptically polarized extraordinary electromagnetic mode [40. Furthermore, the electron-1|2 spin is responsible for a new perpendicularly propagating (with respect to the magnetic field direction) electromagnetic spin wave, which can be excited by intense neutrino bursts in supernovae. The newly derived nonlinear EMHD and Hall-MHD equations can be used for investigating the multi-dimensional linear and nonlinear electromagnetic waves in quantum plasmas. Finally, we have also presented theoretical and numerical studies of stimulated Raman and Brillouin scattering instabilities of a large amplitude electromagnetic wave, and the trapping of arbitrary large amplitude circularly polarized EM waves in a fully nonlinear electron density hole in an unmagnetized quantum plasma. It is expected that localized nanostructures can transport electromagnetic wave energy over nanoscales in laboratory and astrophysical dense plasmas with degenerate electrons.

The field of the nonlinear quantum plasma physics is extremely rich and vibrant today, and it holds a great promise of providing new practical technologies. For example, the plasma assisted carbon nanostructures and nanomaterials are the future of nanotechnologies, so are the new radiation sources in the x-rays and gamma-rays regimes. In such circumstances, one should have a complete understanding of the fundamentals of collective nonlinear interactions (e.g. intense high-order harmonic generation of ultrashort laser pulses from laser-irradiated dense plasma surfaces) in quantum plasmas. Furthermore, in magnetars, and in the next generation intense laser-solid density plasma interaction experiments, one would certainly have degenerate positrons, besides degenerate electrons. The physics of dense quantum magnetoplasmas with degenerate electron-positron pairs is expected to be quite different than what has been described in this paper. The reason is the complex nonlinear dynamics of the electron-positron pairs, which would have relativistic velocities in a dense magnetoplasma. Accordingly, we should develop new theories involving relativistic kinetic and quantum relativistic magnetohydrodynamic equations that include quantum relativistic effects 218, 219, 220], electromagnetic forces, angular momentum spin and nonlinear effects on equal footings. A detailed 
analysis of such theories would provide us a guide line for understanding the origin of localized high-energy radiation and other complex phenomena (e.g. the formation of structures) from astrophysical settings and future laboratory experiments aiming to model astrophysical scenarios.

Acknowledgements

One of the authors (P K Shukla) acknowledges the benefit of useful discussions with Academician professor Vladimir Fortov. We greately appreciate our invaluable collaborations with Lennart Stenflo, Gert Brodin, Mattias Marklund, Fernando Haas and Nitin Shukla.

This research was partially supported by the Deutsche Forschungsgemeinschaft (Bonn, Germany) through the project SH21/3-1 of the Research Unit 1048, and by the Swedish Research Council (VR). 


\section{APPENDIX A: DERIVATION OF THE VLASOV EQUATION FROM THE WIGNER EQUATION}

We here show that the Wigner equation converges to the Vlasov equation in the classical limit $\hbar \rightarrow 0$. The Wigner equation reads

$$
\frac{\partial f}{\partial t}+\mathbf{v} \cdot \nabla f=-\frac{i e m_{e}^{3}}{(2 \pi)^{3} \hbar^{4}} \iint d^{3} \lambda d^{3} v^{\prime} \exp \left[i \frac{m_{e}}{\hbar}\left(\mathbf{v}-\mathbf{v}^{\prime}\right) \cdot \boldsymbol{\lambda}\right]\left[\phi\left(\mathbf{x}+\frac{\boldsymbol{\lambda}}{2}, t\right)-\phi\left(\mathbf{x}-\frac{\boldsymbol{\lambda}}{2}, t\right)\right] f\left(\mathbf{x}, \mathbf{v}^{\prime}, t\right) .
$$

By the change of variables $\boldsymbol{\lambda}=\hbar \boldsymbol{\xi} / m_{e}$ we have $d^{3} \lambda=\hbar^{3} d^{3} \xi / m_{e}^{3}$ and Eq. A1 takes the form

$$
\frac{\partial f}{\partial t}+\mathbf{v} \cdot \nabla f=-\frac{i e}{(2 \pi)^{3} \hbar} \iint d^{3} \xi d^{3} v^{\prime} \exp \left[i\left(\mathbf{v}-\mathbf{v}^{\prime}\right) \cdot \boldsymbol{\xi}\right]\left[\phi\left(\mathbf{x}+\frac{\hbar \boldsymbol{\xi}}{2 m_{e}}, t\right)-\phi\left(\mathbf{x}-\frac{\hbar \boldsymbol{\xi}}{2 m_{e}}, t\right)\right] f\left(\mathbf{x}, \mathbf{v}^{\prime}, t\right) .
$$

Assuming that $\hbar / m_{e}$ is small and Taylor expanding $\phi$ up to the third order around $\mathbf{x}$,

$$
\phi\left(\mathrm{x} \pm \frac{\hbar \boldsymbol{\xi}}{2 m_{e}}, t\right) \approx \phi(\mathrm{x}, t) \pm \frac{\hbar}{2 m_{e}}(\boldsymbol{\xi} \cdot \nabla) \phi(\mathrm{x}, t)+\frac{\hbar^{2}}{8 m_{e}^{2}}(\boldsymbol{\xi} \cdot \nabla)^{2} \phi(\mathrm{x}, t) \pm \frac{\hbar^{3}}{48 m_{e}^{3}}(\boldsymbol{\xi} \cdot \nabla)^{3} \phi(\mathrm{x}, t),
$$

we have

$$
\frac{\partial f}{\partial t}+\mathbf{v} \cdot \nabla f \approx-\frac{i e}{(2 \pi)^{3} m_{e}} \iint d^{3} \xi d^{3} v^{\prime} \exp \left[i\left(\mathbf{v}-\mathbf{v}^{\prime}\right) \cdot \boldsymbol{\xi}\right]\left[\boldsymbol{\xi} \cdot \nabla \phi(\mathrm{x}, t)+\frac{\hbar^{2}}{24 m_{e}^{2}}(\boldsymbol{\xi} \cdot \nabla)^{3} \phi(\mathrm{x}, t)\right] f\left(\mathbf{x}, \mathbf{v}^{\prime}, t\right) .
$$

By the identity

$$
\exp \left[i\left(\mathbf{v}-\mathbf{v}^{\prime}\right) \cdot \boldsymbol{\xi}\right] \boldsymbol{\xi}=i \nabla_{\mathbf{v}^{\prime}} \exp \left[i\left(\mathbf{v}-\mathbf{v}^{\prime}\right) \cdot \boldsymbol{\xi}\right]
$$

where $\nabla_{\mathbf{v}^{\prime}}=\widehat{\mathbf{x}} \partial / \partial v_{x}^{\prime}+\widehat{\mathbf{y}} \partial / \partial v_{y}^{\prime}+\widehat{\mathbf{z}} \partial / \partial v_{z}^{\prime}$, we have

$$
\frac{\partial f}{\partial t}+\mathbf{v} \cdot \nabla f=\frac{e}{(2 \pi)^{3} m_{e}} \iint d^{3} \xi d^{3} v^{\prime}\left\{\exp \left[i\left(\mathbf{v}-\mathbf{v}^{\prime}\right) \cdot \boldsymbol{\xi}\right]\left[\left(\overleftarrow{\nabla}_{\mathbf{v}^{\prime}} \cdot \vec{\nabla}\right)-\frac{\hbar^{2}}{24 m_{e}^{2}}\left(\overleftarrow{\nabla}_{\mathbf{v}^{\prime}} \cdot \vec{\nabla}\right)^{3}\right] \phi(\mathbf{x}, t)\right\} f\left(\mathbf{x}, \mathbf{v}^{\prime}, t\right)
$$

where the arrows indicate the direction of operation of the nabla operators.

Integration by parts in $\mathbf{v}^{\prime}$ space, and using that $f \rightarrow 0$ for $\left|\mathbf{v}^{\prime}\right| \rightarrow \infty$, now yields

$$
\frac{\partial f}{\partial t}+\mathbf{v} \cdot \nabla f=-\frac{e}{(2 \pi)^{3} m_{e}} \iint d^{3} \xi d^{3} v^{\prime} \exp \left[i\left(\mathbf{v}-\mathbf{v}^{\prime}\right) \cdot \boldsymbol{\xi}\right]\left\{\phi(\mathbf{x}, t)\left[\left(\overleftarrow{\nabla} \cdot \vec{\nabla}_{\mathbf{v}^{\prime}}\right)-\frac{\hbar^{2}}{24 m_{e}^{2}}\left(\overleftarrow{\nabla} \cdot \vec{\nabla}_{\mathbf{v}^{\prime}}\right)^{3}\right] f\left(\mathbf{x}, \mathbf{v}^{\prime}, t\right)\right\}
$$

Now the integration in $\boldsymbol{\xi}$ space can formally be performed, with the result

$$
\frac{\partial f}{\partial t}+\mathbf{v} \cdot \nabla f=-\frac{e}{m_{e}} \int d^{3} v^{\prime} \delta\left(\mathbf{v}-\mathbf{v}^{\prime}\right)\left\{\phi(\mathbf{x}, t)\left[\left(\overleftarrow{\nabla} \cdot \vec{\nabla}_{\mathbf{v}^{\prime}}\right)-\frac{\hbar^{2}}{24 m_{e}^{2}}\left(\overleftarrow{\nabla} \cdot \vec{\nabla}_{\mathbf{v}^{\prime}}\right)^{3}\right] f\left(\mathbf{x}, \mathbf{v}^{\prime}, t\right)\right\}
$$

where the identity

$$
\int d^{3} \xi \exp \left[i\left(\mathbf{v}-\mathbf{v}^{\prime}\right) \cdot \xi\right]=(2 \pi)^{3} \delta\left(\mathbf{v}-\mathbf{v}^{\prime}\right)
$$

was used and where $\delta$ is Dirac's delta function. Finally, integration over $\mathbf{v}^{\prime}$ space yields

$$
\frac{\partial f}{\partial t}+\mathbf{v} \cdot \nabla f=-\frac{e}{m_{e}}\left\{\phi(\mathbf{x}, t)\left[\left(\overleftarrow{\nabla} \cdot \vec{\nabla}_{\mathbf{v}}\right)-\frac{\hbar^{2}}{24 m_{e}^{2}}\left(\overleftarrow{\nabla} \cdot \vec{\nabla}_{\mathbf{v}}\right)^{3}\right] f(\mathbf{x}, \mathbf{v}, t)\right\}
$$

In the limit $\hbar \rightarrow 0$, we recover the Vlasov equation

$$
\frac{\partial f}{\partial t}+\mathbf{v} \cdot \nabla f=-\frac{e}{m_{e}} \nabla \phi(\mathrm{x}, t) \cdot \nabla_{\mathbf{v}} f(\mathbf{x}, \mathbf{v}, t) .
$$




\section{APPENDIX B: DERIVATION OF THE DISPERSION RELATION FOR THE WIGNER-POISSON SYSTEM}

We here present a derivation of the dispersion relation for electrostatic waves in a degenerate quantum plasma, governed by the Wigner-Poisson system of equations. The linearized Wigner-Poisson system of equations reads

$$
\frac{\partial f_{1}}{\partial t}+\mathbf{v} \cdot \nabla f_{1}=-\frac{i e m_{e}^{3}}{(2 \pi)^{3} \hbar^{4}} \iint d^{3} \lambda d^{3} v^{\prime} \exp \left[i \frac{m_{e}}{\hbar}\left(\mathbf{v}-\mathbf{v}^{\prime}\right) \cdot \boldsymbol{\lambda}\right]\left[\phi_{1}\left(\mathbf{x}+\frac{\boldsymbol{\lambda}}{2}, t\right)-\phi_{1}\left(\mathbf{x}-\frac{\boldsymbol{\lambda}}{2}, t\right)\right] f_{0}\left(\mathbf{v}^{\prime}\right),
$$

and

$$
\nabla^{2} \phi_{1}=4 \pi e \int f_{1} d^{3} v
$$

where $f_{0}$ denotes the background distribution function, and $f_{1}$ and $\phi_{1}$ denote the perturbed distribution function and electrostatic potential, respectively. Fourier transforming $(\mathrm{B} 1)-(\mathrm{B} 2)$ in space and Laplace transforming them in time, we obtain

$$
\begin{gathered}
(\omega-\mathbf{k} \cdot \mathbf{v}) f_{1}=\frac{e m_{e}^{3}}{(2 \pi)^{3} \hbar^{4}} \iint d^{3} \lambda d^{3} v^{\prime} \exp \left[i \frac{m_{e}}{\hbar}\left(\mathbf{v}-\mathbf{v}^{\prime}\right) \cdot \boldsymbol{\lambda}\right]\left[e^{i \mathbf{k} \cdot \boldsymbol{\lambda} / 2}-e^{-i \mathbf{k} \cdot \boldsymbol{\lambda} / 2}\right] f_{0}\left(\mathbf{v}^{\prime}\right) \phi_{1}(\omega, \mathbf{k}), \\
k^{2} \phi_{1}=-4 \pi e \int f_{1} d^{3} v
\end{gathered}
$$

Rewriting (B3) as

$$
\begin{aligned}
& (\omega-\mathbf{k} \cdot \mathbf{v}) f_{1}=\frac{i e m_{e}^{3}}{(2 \pi)^{3} \hbar^{4}} \iint d^{3} \lambda d^{3} v^{\prime}\left\{\exp \left[\frac{m_{e}}{\hbar}\left(\mathbf{v}-\mathbf{v}^{\prime}\right) \cdot \boldsymbol{\lambda}+i \mathbf{k} \cdot \boldsymbol{\lambda} / 2\right]\right. \\
& \left.-\exp \left[i \frac{m_{e}}{\hbar}\left(\mathbf{v}-\mathbf{v}^{\prime}\right) \cdot \boldsymbol{\lambda}-i \mathbf{k} \cdot \boldsymbol{\lambda} / 2\right]\right\} f_{0}\left(\mathbf{v}^{\prime}\right) \phi_{1}(\omega, \mathbf{k})
\end{aligned}
$$

and performing the integration over $\boldsymbol{\lambda}$ space, we have

$$
(\omega-\mathbf{v} \cdot \mathbf{k}) f_{1}=\frac{e m_{e}^{3}}{\hbar^{4}} \int d^{3} v^{\prime}\left\{\delta\left[\frac{m_{e}}{\hbar}\left(\mathbf{v}-\mathbf{v}^{\prime}\right)+\frac{\mathbf{k}}{2}\right]-\delta\left[\frac{m_{e}}{\hbar}\left(\mathbf{v}-\mathbf{v}^{\prime}\right)-\frac{\mathbf{k}}{2}\right]\right\} f_{0}\left(\mathbf{v}^{\prime}\right) \phi_{1}(\omega, \mathbf{k})
$$

where $\delta$ is the Dirac delta function. Now, the integration can be performed over $\mathbf{v}^{\prime}$ space, obtaining the result

$$
(\omega-\mathbf{k} \cdot \mathbf{v}) f_{1}=\frac{e}{\hbar}\left[f_{0}\left(\mathbf{v}+\frac{\hbar \mathbf{k}}{2 m_{e}}\right)-f_{0}\left(\mathbf{v}-\frac{\hbar \mathbf{k}}{2 m_{e}}\right)\right] \phi_{1}(\omega, \mathbf{k}) .
$$

Solving for $f_{1}$ in $(\mathrm{B} 7)$ and inserting the result in $(\mathrm{B} 4)$, we obtain the dispersion relation

$$
1-\frac{4 \pi e^{2} k^{2}}{\hbar} \int\left[\frac{f_{0}\left(\mathbf{v}+\frac{\hbar \mathbf{k}}{2 m_{e}}\right)}{(-\omega+\mathbf{k} \cdot \mathbf{v})}-\frac{f_{0}\left(\mathbf{v}-\frac{\hbar \mathbf{k}}{2 m_{e}}\right)}{(-\omega+\mathbf{k} \cdot \mathbf{v})}\right] d^{3} v=0
$$

Suitable changes of variables in the two terms now gives

$$
1-\frac{4 p i e^{2} k^{2}}{\hbar} \int\left[\frac{1}{\left[-\omega+\mathbf{k} \cdot\left(\mathbf{u}-\frac{\hbar \mathbf{k}}{2 m_{e}}\right)\right]}-\frac{1}{\left[-\omega+\mathbf{k} \cdot\left(\mathbf{u}+\frac{\hbar \mathbf{k}}{2 m_{e}}\right)\right]}\right] f_{0}(\mathbf{u}) d^{3} u=0,
$$

which can be rewritten as

$$
1-\frac{4 \pi e^{2}}{m_{e}} \int \frac{f_{0}(\mathbf{u})}{(\omega-\mathbf{k} \cdot \mathbf{u})^{2}-\frac{\hbar^{2} k^{4}}{4 m_{e}^{2}}} d^{3} u=0 .
$$

The dispersion relation (B10 was also derived by Bohm and Pines [3] using a series of canonical transformations of the Hamiltonian of the system; see for example equation (57) in their paper. We now choose a coordinate system such that the $x$ axis is aligned with the wave vector $\mathbf{k}$. Then, $\mathrm{B} 10$ takes the form

$$
1-\frac{4 \pi e^{2}}{m_{e}} \int \frac{f_{0}(\mathbf{u})}{\left(\omega-k u_{x}\right)^{2}-\frac{\hbar^{2} k^{4}}{4 m_{e}^{2}}} d^{3} u=0 .
$$


We next consider a dense plasma with degenerate electrons in the zero temperature limit. Then, the background distribution function takes the simple form

$$
f_{0}=\left\{\begin{array}{cc}
2\left(\frac{m_{e}}{2 \pi \hbar}\right)^{3}, & |\mathbf{u}| \leq V_{F e} \\
0, & \text { elsewhere }
\end{array}\right.
$$

where $V_{F e}=\left(2 \mathcal{E}_{F e} / m_{e}\right)^{1 / 2}$ is the speed of an electron on the Fermi surface, and $\mathcal{E}_{F e}=\left(3 \pi^{2} n_{0}\right)^{2 / 3} \hbar^{2} /\left(2 m_{e}\right)$ is the Fermi energy. The integration in (B11) can be performed over velocity space perpendicular to $u_{x}$, using cylindrical coordinate in $u_{y}$ and $u_{z}$, obtaining the result

$$
1=\frac{4 \pi e^{2}}{m_{e}} \int \frac{F_{0}\left(u_{x}\right)}{\left(\omega-k u_{x}\right)^{2}-\frac{\hbar^{2} k^{4}}{4 m_{e}^{2}}} d u_{x}
$$

where

$$
F_{0}\left(u_{x}\right)=\iint f_{0}(\mathbf{u}) d u_{y} d u_{z}=2 \pi \int_{0}^{\sqrt{V_{F e}^{2}-u_{x}^{2}}} 2\left(\frac{m_{e}}{2 \pi \hbar}\right)^{3} u_{\perp} d u_{\perp}=\left\{\begin{array}{cl}
2 \pi\left(\frac{m_{e}}{2 \pi \hbar}\right)^{3}\left(V_{F e}^{2}-u_{x}^{2}\right), & \left|u_{x}\right| \leq V_{F e} \\
0, & \text { elsewhere. }
\end{array}\right.
$$

Equation B13 can be written as

$$
1=\frac{8 \pi^{2} e^{2}}{m_{e}}\left(\frac{m_{e}}{2 \pi \hbar}\right)^{3} \int_{-V_{F e}}^{V_{V e}} \frac{V_{F e}^{2}-u_{x}^{2}}{\left(\omega-k u_{x}\right)^{2}-\frac{\hbar^{2} k^{4}}{4 m_{e}^{2}}} d u_{x}=\frac{3 \omega_{p e}^{2}}{4 V_{F e}^{3}} \int_{-V_{F e}}^{V_{V e}} \frac{V_{F e}^{2}-u_{x}^{2}}{\left(\omega-k u_{x}\right)^{2}-\frac{\hbar^{2} k^{4}}{4 m_{e}^{2}}} d u_{x} .
$$

First, in the limit $\hbar k / m_{e} \rightarrow 0$, we have from B15

$$
1+\frac{3 \omega_{p e}^{2}}{k^{2} V_{F e}^{2}}\left(1-\frac{\omega}{2 k V_{F e}} \log \left|\frac{\omega+k V_{F e}}{\omega-k V_{F e}}\right|\right)=0
$$

where we have assumed that $\omega$ is real and $\omega / k>V_{F e}$.

Expanding $(\mathrm{B} 16)$ for small wavenumbers up to terms containing $k^{2}$, we have

$$
\omega^{2}=\omega_{p e}^{2}+\frac{3}{5} k^{2} V_{F e}^{2} .
$$

Second, for non-zero $\hbar k^{2} / m_{e}$, we have from $\mathrm{B} 15$ the dispersion relation

$$
\begin{array}{r}
1+\frac{3 \omega_{p e}^{2}}{4 k^{2} V_{F e}^{2}}\left\{2-\frac{m_{e}}{\hbar k V_{F e}}\left[V_{F e}^{2}-\left(\frac{\omega}{k}+\frac{\hbar k}{2 m_{e}}\right)^{2}\right] \log \left|\frac{\frac{\omega}{k}-V_{F e}+\frac{\hbar k}{2 m_{e}}}{\frac{\omega}{k}+V_{F e}+\frac{\hbar k}{2 m_{e}}}\right|\right. \\
\left.+\frac{m_{e}}{\hbar k V_{F e}}\left[V_{F e}^{2}-\left(\frac{\omega}{k}-\frac{\hbar k}{2 m_{e}}\right)^{2}\right] \log \left|\frac{\frac{\omega}{k}-V_{F e}-\frac{\hbar k}{2 m_{e}}}{\frac{\omega}{k}+V_{F e}-\frac{\hbar k}{2 m_{e}}}\right|\right\}=0 .
\end{array}
$$

Expanding (B18) for small wavenumbers up to terms containing $k^{4}$, we obtain

$$
\omega^{2} \approx \omega_{p e}^{2}+\frac{3}{5} k^{2} V_{F e}^{2}+(1+\alpha) \frac{\hbar^{2} k^{4}}{4 m_{e}^{2}},
$$

where $\alpha=(48 / 175) m_{e}^{2} V_{F e}^{4} / \hbar^{2} \omega_{p e}^{2} \approx 2.000\left(n_{0} a_{0}^{3}\right)^{1 / 3}$ and $a_{0}=\hbar^{2} / m_{e} e^{2} \approx 53 \times 10^{-10} \mathrm{~cm}$ is the Bohr radius. For a typical metal such as gold, which has a free electron number density of $n_{0}=5.9 \times 10^{22} \mathrm{~cm}^{-3}$, we would have $\alpha \approx 0.4$. For the free electron density in semiconductors, which is many orders of magnitude less than in metals, $\alpha$ is much smaller and can safely be dropped compared to unity. 
[1] Klimontovich Y L and Silin V P Doklady Akad. Nauk S. S. S. R. 82361 (1952); JFTF (Journal Experimental Teoreticheskoi Fisiki) 23151 (1952)

[2] Bohm D Phys. Rev. 85166 (1952)

[3] Bohm D and Pines D Phys. Rev. 92609 (1953)

[4] Pines D J. Nucl. Energy: Part C: Plasma Phys. 25 (1961)

[5] Bohm D and Pines D in Plasma Physics, Ed. J. E. Drummond (McGraw-Hill, New York, 1961) Chap. 2 pp. $35-87$

[6] Bonitz M et al. J. Phys. A: Math. Gen. 365921 (2003)

[7] Manfredi G Fields Inst. Commun. 46263 (2005)

[8] Bransden B H and Joachain C J Quantum Mechanics (2nd Edition) (Pearson Education Limited, Essex, England, 2000)

[9] Fortov V E and Iakubov I T The Physics of Ion-Ideal Plasma (World Scientific, Singapore, 2000)

[10] Lee T D Astrophys. J. 111625 (1950)

[11] Hubbard W B Astrophys. J. 146858 (1966)

[12] Lampe M Phys. Rev. 170306 (1968)

[13] Azechi H et al. Laser Part. Beams 9, 193 (1991)

[14] Azechi H et al. Plasma Phys. Control. Fusion 48, B267 (2006)

[15] Son S and Fisch N J Phys. Rev. Lett. 95225002 (2005)

[16] Wigner E Phys. Rev. 40749 (1932)

[17] Moyal I E Proc. Cambridge Phil. Soc. 4599 (1949)

[18] Anderson D et al. Phys. Rev. E 65046417 (2002).

[19] Tsintsadze N L and Tsintsadze L N arXiv:0903.5368v1 [physics.plasm-ph] 31 Mar 2009

[20] Gardner C L and Ringhofer C Phys. Rev. E 53157 (1996)

[21] Manfredi G and Haas F Phys. Rev. B 64075316 (2001)

[22] Shukla P K and Eliasson B Phys. Rev. Lett. 96245001 (2006)

[23] Shukla P K Phys. Lett. A 352242 (2006)

[24] Shukla P K and Eliasson B New J. Phys. 998 (2007)

[25] Maafa N Phys. Scripta 48351 (1993)

[26] Melrose D B Quantum Plasmadynamics: Unmagnetized Plasmas (Springer, Berlin, 2007)

[27] Lifshitz E M and Pitaevskii L P Physical Kinetics (Butterworth-Heinemann, Oxford, 1981)

[28] Nozieres P and Pines D The Theory of Quantum Liquids (Perscus Books, Cambridge, 1999) p 279

[29] Kremp D et al. Phys. Rev. E 604725 (1999)

[30] Bonitz M Quantum Kinetic Theory (B G Teubner, Stuttgart, 1998)

[31] Kremp D, Schlanges M and Kraeft W D Quantum Statistics of Nonideal Plasmas (Springer, Berlin, 2005)

[32] Uhlenbeck G E and Goudsmit S Naturwissenschaften 47953 (1025); Nature (London) 117264 (1926)

[33] Bohm D Quantum Theory (Dover, Prentice-Hall, New York, 1952)

[34] Sasabe S and Tsuchiya K Phys. Lett. A 372381 (2008)

[35] Landau L D and Lifshitz Quantum Mechanics 3rd edition (Pergamon, Oxford, 1977)

[36] Steinberg M Thermodynamics and Kinetics of a Magnetized Quantum Plasma (Logos, Berlin, 2000)

[37] John P Contrib. Plasma Phys. 33488 (2006)

[38] Shukla P K and Stenflo L J. Plasma Phys. 72605 (2006)

[39] Shukla P K et al. Phys. Plasmas 13112111 (2006)

[40] Shukla P K Phys. Lett. A 369312 (2007)

[41] Lundin J et al. Phys. Plasmas 14062112 (2007)

[42] Misra A P Phys. Plasmas 14064501 (2007)

[43] Khan S A and Saleem H Phys. Plasmas 16052109 (2009)

[44] Madelung von E Z. f. Physik 40332 (1926)

[45] Takabayashi T Prog. Theor. Phys. 8143 (1952)

[46] Takabayashi T Prog. Theor. Phys. 14283 (1955)

[47] Takabayashi T Prog. Theor. Phys. 70, 1 (1983)

[48] Bohm D, Schiller R, Tiomno J Suppl. Nuovo Cimento 148 (1955)

[49] Bohm D, Schiller R Suppl. Nuovo Cimento 1, 67 (1955)

[50] Janossy L and Ziegler-Naray M Acta Phys. Hung. 2023 (1965)

[51] Ghosh S K and Deb M M Phys. Rep. 921 (1982)

[52] Takabayashi T Prog. Theor. Phys. 9187 (1953)

[53] Takabayashi T Prog. Theor. Phys. 13, 222 (1955)

[54] Takabayashi T Nuovo Cimento 3, 233 (1956)

[55] Takabayashi T Prog. Theor. Phys. Suppl. 4, 1 (1957)

[56] Bialynicki-Birula I Acta Phys. Polonica B 261201 (1995)

[57] Gardner C L SIAM J. Appl. Math. 54409 (1994)

[58] Loffredo M and Morato L Nuovo Cimento Soc. Ital Fis. B 108B 205 (1993)

[59] Feynman R P Statistical Mechanics, A Set of of Lectures (Benjamin, Reading, 1972)

[60] Domps A et al. Phys. Rev. Lett. 80, 5520 (1998) 
[61] Haas F, Garcia L G, Goedert J and Manfredi G Phys. Plasmas 103858 (2003)

[62] Eliasson B and Shukla P K J. Plasma Phys. 74, 581 (2008).

[63] Haas F Phys. Plasmas 12062117 (2005)

[64] Shukla P K and Stenflo L Phys. Plasmas 13044505 (2006)

[65] Shukla P K and Eliasson B Phys. Rev. Lett. 99096401 (2007)

[66] Shaikh D and Shukla P K Phys. Rev. Lett. 99125002 (2007)

[67] Shaikh D and Shukla P K New J. Phys. 10083007 (2008)

[68] Bershadskii A Phys. Lett. A 3722741 (2008)

[69] Eliasson B and Shukla P K Phys. Scr. 78025503 (2008)

[70] Oraevsky V N and Semikoz V B Phys. Atomic Nuclei 66466 (2003) [Yadernaya Fizika, 66, 494 (2003)]; Oraevsky et al. arXiv:hep-ph/0203020v1 2 Mar 2002

[71] Marklund M and Brodin G Phys. Rev. Lett. 98025001 (2007)

[72] Brodin G and Marklund M Phys. Rev. E 76 055403(R) (2007)

[73] Shukla P K and Stenflo L J. Plasma Phys. 74719 (2008)

[74] Shukla P K Nature Phys. 592 (2009)

[75] Oraevsky V N and Semikoz V B Astropart. Phys. 18261 (2002)

[76] Hu S X and Keitel C H Phys. Rev. Lett. 834709 (1999)

[77] Andreev A V JETP Lett. 72238 (2000)

[78] Mourou G et al. Rev. Mod. Phys. 78309 (2006)

[79] Marklund M and Shukla P K Rev. Mod. Phys. 78591 (2006)

[80] Salamin Y A et al. Phys. Rep. 42741 (2006)

[81] Malkin V M et al. Phys. Rev. E 75026404 (2007)

[82] Kritcher A L et al. Science 32269 (2008)

[83] Hartemann F V et al. Phys. Rev. Lett. 100125001 (2008)

[84] Lee H J et al. Phys. Rev. Lett. 102115001 (2009)

[85] Norreys P A Phys. Plasmas 16041002 (2009)

[86] Drake R P Phys. Plasmas 16055501 (2009).

[87] van Horn H M Science 252384 (1991)

[88] Guillot T Science 28672 (1999)

[89] Fortney J J et al. Phys. Plasmas 16041003 (2009)

[90] Meszaros P High Energy Radiation from Magnetized Neutron Stars (University of Chicago Press, Chicago, 1992)

[91] Gurevich A V, Beskin V S, and Istomin Ya N Physics of the Pulsar Magnetosphere (Cambridge University Press, Cambridge, 1993)

[92] Craighead H G Science 2901532 (2000)

[93] Opher M et al. Phys. Plasmas 8, 2454 (2001)

[94] Shapiro S L and Teukolsky S A Black Holes, White Dwarfs, and Neutron Stars: The Physics of Compact Objects (WileyVCH, Weinheim, 2004)

[95] Benvenuto O G and De Vito M A Mon. Not. R. Astron. Soc. 362, 891 (2005).

[96] Chabrier G et al. J. Phys.: Condens. Matter 149133 (2002)

[97] Chabrier G et al. J. Phys. A: Math. Gen. 394411 (2006)

[98] Lai D Rev. Mod. Phys. 73629 (2001); Harding A K and Lai D Rep. Prog. Phys. 692631 (2006)

[99] Lau Y Y et al. Phys. Rev. Lett. 661446 (1991)

[100] Ang L K et al. Phys. Rev. Lett. 91208303 (2003)

[101] Ang L K, Lau Y Y and Kwan T J T IEEE Trans. Plasma Sci. 32410 (2004)

[102] Ang L K, Koh W S, Lau Y Y and Kwan T J T Phys. Plasmas 13056701 (2006)

[103] Ang L K and Zhang P Phys. Rev. Lett. 98, 164802 (2007)

[104] Shukla P K and Eliasson B Phys. Rev. Lett. 100036801 (2008)

[105] Shpatakovskaya G V JETP 102466 (2006)

[106] Barnes W L et al. Nature (London) 424824 (2003)

[107] Chang D E et al. Phys. Rev. Lett. 97053002 (2006)

[108] Marklund M et al. Europhys. Lett. 84, 17006 (2008)

[109] Markovich A et al. Semiconductor Equation (Springer, Vienna, 1990)

[110] Abrahams E et al. Rev. Mod. Phys. 73251 (2001)

[111] Magnus W C J and Schoemaker W J Quantum Transport in Sub-Micron Devices (Springer, Berlin, 2002)

[112] Crouseilles N, Hervieux P A, and Manfredi G Phys. Rev. B 78155412 (2008)

[113] Becker K H Schoenbach K H and Eden J G J. Phys. D: Appl. Phys. 39 R55 (2006)

[114] Serbeto A, Mendonça J T, Tsui K H and Bonifacio R Phys. Plasmas 15013110 (2008)

[115] Piovella N et al. Phys. Rev. Lett. 100, 044801 (2008)

[116] Lindl J Phys. Plasmas 2, 3933 (1995)

[117] Tabak M et al. Phys. Plasmas 1, 1626 (1994); ibid. 12057305

[118] Glenzer S H et al. Phys. Rev. Lett. 98065002 (2007)

[119] Benney D J and Newell A C J. Math. Phys. 46, 81 (1967)

[120] Benjamin T B Proc. R. Soc. London A 29959 (1967)

[121] Scott A C et al Proc. IEEE 661444 (1973) 
[122] Hasegawa A and Tappert F Appl. Phys. Lett. 23 (1973)

[123] Agrawal G P Nonlinear Fiber Optics (Academic Press, San Diego, 2006)

[124] Wan W, Jia S, and Fleisher J W Nature Phys. 346 (2007)

[125] Karpman V I and Krushkal E M Soviet Phys. JETP 28277 (1969)

[126] Karpman V I Plasma Phys. 13477 (1971)

[127] Zakharov V E Soviet Phys. JETP 35908 (1972)

[128] Karpman V I Nonlinear Waves in Dispersive Media (Pergamon, New York, 1975)

[129] Schamel H and Shukla P K Phys. Rev. Lett. 36968 (1976)

[130] Shukla P K Nature (London) 274874 (1978)

[131] Sulem C and Sulem P L The Nonlinear Schrödinger Equation: Self-Focusing and Collapse (Springer, Berlin, 1999)

[132] Fedele R Phys. Scr. 65502 (2002)

[133] Dauxois T and Peyrard M Physics of Solitons (Cambridge University Press, Cambridge, 2006)

[134] Barenghi et al (Eds) Quantized Vortex Dynamics and Superfluid Turbulence (Springer, Berlin, 2001)

[135] Bewley G P, Lathrop D P and Sreenivasan K R Nature (London) 441588 (2006)

[136] Bose S Z. Phys. 26178 (1924)

[137] Einstein A Sitzungsber. K. Preuss Akad. Wiss. Phys. Math. K1. 261 (1924)

[138] Gross E P Il Nuovo Cimento 20454 (1961);

[139] Pitaevskii L P Sov. Phys. JETP 13451 (1961)

[140] Pitaevskii L P and Stringari S Bose-Einstein Condensation (Clarendon, Oxford, 2003)

[141] Dalfovo et al. Rev. Mod. Phys. 71463 (1999)

[142] O'Dell D H J et al. Phys. Rev. Lett. 92250401 (2004)

[143] Pauli W Z. Physik 32111 (1925); Rev. Mod. Phys. 13203 (1941)

[144] Berestetskii B V, Lifshitz E M and Pitaevskii L P Quantum Electrodynamics (Butterworth-Heinemann, Oxford, 1999) p 123

[145] Brodin G and Marklund M New J. Phys. 9277 (2007)

[146] Dvornikov M arXiv:0902.4596v1 [physics.plasm-ph] 26 Feb 2009

[147] Kolomeisky E B et al. Phys. Rev. Lett. 851146 (2000)

[148] Ivonin I A JETP 851233 (1997)

[149] Ivonin I A, Pavlenko V P and Persson H Phys. Rev. E 60492 (1999)

[150] Whitham G B Linear and Nonlinear Waves (John Wiley \& Sons, New York, 1999)

[151] Sagdeev R Z in Reviews of Plasma Physics edited by Leontovich M A (Consultants Bureau, New York, 1966) Vol 4 p 23

[152] Sagdeev R Z Rev. Mod. Phys. 5111 (1979)

[153] Shukla P K and Yu M Y J. Math. Phys. 192506 (1978); Yu M Y et al. Phys. Fluids 232146 (1980)

[154] Gottlieb D and Orszag S A Numerical Analysis of Spectral Methods (SIAM, Philadelphia 1977)

[155] Kolmogorov A N Dokl. Akad, Nauk SSR 30301 (1941); 31438 (1941)

[156] Lesieur M Turbulence in Fluids (Kluwer, Dordrecht 1990)

[157] Frisch U Turbulence (Cambridge University Press, Cambridge, England 1995)

[158] Eyink G L and Sreenivasan K R Rev. Mod. Phys. 7887 (2006)

[159] Iroshnikov P Sov. Astron. 7566 (1963)

[160] Kraichnan R H Phys. Fluids 81385 (1965)

[161] Larichev V D and McWilliams J C Phys. Fluids A 3938 (1991)

[162] Scott R K Phys. Rev. E 75046301 (2007)

[163] Paoletti M S et al Phys. Rev. Lett. 101154501 (2008)

[164] Eliasson B and Shukla P K Phys. Rep. 422225 (2006)

[165] Haas F, Eliasson B, Shukla P K and Manfredi G Phys. Rev. E 780564407 (2008)

[166] Kruer W L, Dawson J M and Sudan R N Phys. Rev. Lett. 23838 (1969)

[167] Albrecht-Marc M et al. Phys. Plasmas 14072704 (2007)

[168] Luque et al. Phys. Lett. A 324185 (2004)

[169] Biermann P Z. Naturforsch. A 565 (1950)

[170] Weibel E S Phys. Rev. Lett. 283 (1959)

[171] Gruzinov A Astrophys. J. 563, L15 (2001)

[172] Schlickeiser R and Shukla P K Astrophys. J. 599 L57 (2003)

[173] Karpman V I and Washimi H Sov. Phys. JETP 44528 (1976).

[174] Karpman V I and Washimi H J. Plasma Phys. 18173 (1977)

[175] Gradov O M and Stenflo L Zh. Naturforsch. 35a 461 (1980)

[176] Gradov O M and Stenflo L Phys. Lett. A 95A 233 (1983)

[177] Shukla P K and Yu M Y Plasma Phys. Controll. Fusion 26, 841 (1984)

[178] Shukla P K et al. Phys. Rep. 1381 (1986)

[179] Tsintsadze L N and Shukla P K Phys. Lett. A 18767 (1994)

[180] Tsintsadze L N and Shukla P K J. Plasma Phys. 74, 431 (2008)

[181] Haas F Phys. Plasmas 15022104 (2008)

[182] Haas F and Lazar M Phys. Rev. E 77046404 (2008)

[183] Haas F et al. J Plasma Phys. 750251 (2009)

[184] Pines D, Nozières P The Theory of Quantum Liquids (W. A. Benjamin, New York, 1966) 
[185] Lindhard J, Dan K Vidensk. Selsk. Mat. Fys. Medd. 281 (1954)

[186] Cockayne E and Levine Z H, Phys. Rev. B 74235107 (2006)

[187] Wei M S et al. Phys. Rev. E 70056412 (2004)

[188] Estabrook K Phys. Rev. Lett. 411808 (1978)

[189] Abramowitz M and Stegun I A (eds.), Handbook of Mathematical Functions with Formulas, Graphs and Mathematical Tables (Dover, New York, 1972)

[190] Lewin L Polylogarithms and Associated Functions (North-Holland, New York, 1981)

[191] Ross O Phys. Rev. 1191174 (1960)

[192] Arista N R and Brandt W Phys. Rev. A 291471 (1984)

[193] Leemans W P et al. Phys. Rev. A 461091 (1992)

[194] Fried B D and Conte S D The Plasma Dispersion Function (Academic Press, London, 1961)

[195] Krall N A and Trivelpiece A W Principles of Plasma Physics (McGraw-Hill, New York, 1973)

[196] Davidson R C et al. Phys. Fluids 15317 (1972)

[197] Eliasson B J. Comput. Phys. 2251508 (2007)

[198] Shukla P K, Shukla N and Stenflo L J. Plasma Phys. 75 in press (2009) doi:10.1017/S0022377809008022

[199] Oberman C and Ron A Phys. Rev. 1301291 (1963)

[200] Kelley C Phys. Rev. 134 A641 (1964)

[201] Benford G and Rostoker N Phys. Rev. 181729 (1969)

[202] Kuzelev M V and Rukhadze A A Phys. Usp. 42603 (1999)

[203] Melrose D B and Weise J I Phys. Plasmas 94473 (2002)

[204] Ginzburg V L Propagation of Electromagnetic Waves in Plasma (Gordon and Breach, New York, 1960)

[205] Abdullaev A Sh Sov. J. Plasma Phys. 14214 (1988)

[206] Eliezer S et al. Phys. Plasmas 12052115 (2005)

[207] Boose D and Perez A Phys. Lett. A 234113 (1997)

[208] Kittel C Introduction to Solid State Physics, 7th edition (John Wiley \& Sons, New York, 1996) Chapter 14.

[209] Marklund M et al. Phys. Rev. E 76067401 (2007)

[210] Shaikh D and Shukla P K Phys. Rev. Lett. 102045004 (2009)

[211] Drake J F et al. Phys. Fluids 17778 (1974)

[212] Yu M Y et al. Z. Naturforsch. 29a 1736 (1974)

[213] Stenflo L Phys. Scr. T30 166 (1990)

[214] Sagdeev R Z and Galeev A Nonlinear Plasma Theory (Benjamin, New York, 1969)

[215] Haas F Phys. Plasmas 14042309 (2007)

[216] Haas F and Shukla P K Phys. Rev. E 79077402 (2009).

[217] Marburger J H and Tooper R H Phys. Rev. Lett. 351001 (1975)

[218] Hakim R and Heyvarerts J Phys. Rev. A 181250 (1978)

[219] Shabad A Y and Ginzburg V L Polarization of the Vacuum and a Quantum Relativistic Gas in an External Field (Nova Sci, Pub. Inc, New York, 1991)

[220] Lamata L et al. Phys. Rev. Lett. 98253005 (2007) 